\title{
Ethnobotanical study of medicinal plants in the environs of Tara-gedam and Amba remnant forests of Libo Kemkem District, northwest Ethiopia
}

\author{
Getnet Chekole ${ }^{1 *}$, Zemede Asfaw ${ }^{2}$ and Ensermu Kelbessa ${ }^{2}$
}

\begin{abstract}
Background: Remnant forests found in areas that have long been converted to agricultural landscapes are refuges of wild useful plants; and societies inhabiting them are custodians of rich indigenous botanical knowledge. This study was undertaken to document the medicinal plants used by the people living in and around Tara-gedam and Amba remnant forests, northwestern Ethiopia, together with the associated ethnomedicinal knowledge.
\end{abstract}

Methods: Data were collected from 105 informants through semi-structured interviews, guided field walk, market survey; and analyzed using standard ethnobotanical analytical tools including ranking and comparison.

Results: A total of 163 medicinal plant species in 145 genera and 67 families were recorded among which Zehneria scabra drew the highest community consensus. Seventy-one percent of the medicinal plants were those used for treating human ailments only, 21\% for both human and livestock and 8\% for livestock only. Asteraceae, with 14 species, had the highest number of medicinal plant species. The medicinal plants mainly (79.1\%) belong to the shrub and herb categories and most of them were sourced from the wild habitats. Leaves and fresh plant materials were more frequently used for medicine preparation than other parts. Protected government and church forests as well as tree propagation in nurseries followed by planting them and local practices constitute the major forest conservation efforts that indirectly protect the medicinal plants in the area. Elders and healers knew more about the medicinal plants, their distribution, the local ethnomedicinal practices and knowledge transfer patterns. Though important for the local healthcare system and with potentials for modern drug discovery, both the plants and the knowledge pool are under threat.

Conclusion: The diversity of medicinal plants and the associated indigenous knowledge of Tara-gedam and its environs are of a considerable value to the local community and beyond. There is, therefore, a need for conservation of the vegetation and the medicinal plants along with preservation of the wealth of the indigenous knowledge.

Keywords: Ethiopia, Ethnobotany, Indigenous botanical knowledge, Medicinal plants, Tara-gedam

\section{Introduction}

The relationship between plants and people is studied in ethnobotany, a field focusing on the study of the indigenous knowledge on how plants are perceived, used and managed $[1,2]$. Indigenous knowledge refers to the knowledge, rules, standards, skills and mental sets generated by and kept in custody of local people in a particular area [3]. It is the result of many generations and long years of experiences, careful observations and trial and

\footnotetext{
* Correspondence: gchekole1@gmail.com

'Department of Biology, Woldia University, P.O. Box 400, Woldia, Ethiopia

Full list of author information is available at the end of the article
}

error experiments [4]; and this study focuses on the medicinal plants and the associated ethnomedicinal knowledge in the environs of Tara-gedam forest. The cultural and spiritual identity of indigenous peoples is often linked to intact primary forests with their rich biodiversity [5]. Hence, plant resources possess and preserve cultural heritages, biological information and indigenous knowledge on plant identity and utility [6]. The ethnobotanical literature [7] underlines that both saving plant species and documenting and preserving indigenous knowledge associated with them are fundamental urgent concerns. There are around 6,000 species of vascular 
plants in Ethiopia, out of which more than 14\% are said to have been used as traditional plant medicines (TPMs) [8], while more than 1,000 species have been documented at the National Herbarium (ETH) database. Despite their treasured contributions, in particular in Ethiopia, thus far TPMs have been offered very little attention in modern research and development, while less effort has so far been made to upgrade the traditional herbal medical practices [9]. For the most part, the potential of practitioners of traditional herbal medicine to serve as partners in the process of drug discovery and in providing healthcare services is not equitably acknowledged [10]. Hence, documenting traditional medicinal plants and the related traditional medical knowledge is important in order to facilitate the discovery of new sources of drugs and promote sustainable use of natural resources in Ethiopia [11]. Tara-gedam forest, selected for the study, is among the national priority forest areas in Ethiopia [12] and Amba forest is found adjacent to it. Both these remnant forests are known as species rich forests in Amhara Region, and the nearby local communities are in constant interaction with the plant resources [13,14], particularly so for those living in the forest fringes. Research revealed that urbanization in Ethiopia had tremendous impacts on the useful plants and the practice of traditional medicine [15]. Since Taragedam and Amba forests are found adjacent to the growing Addis Zemen Town, the impacts have already been alluded to by some researchers [14]. The local people, as in other parts of Ethiopia depend on traditional medicine, which mostly relies on medicinal plants, to fulfill their healthcare needs as pointed out by Zegeye [14]. Despite this fact, there are no studies on ethnomedicinal plants and the associated knowledge in the environs of Tara-gedam and Amba forests. Hence, this study was framed with the aim of documenting the medicinal plants and the associated ethnomedicinal knowledge of people living in the environs of Tara-gedam and Amba forests.

\section{Material and methods}

\section{The study area and the demographic background}

The study was conducted in the general environment of Tara-gedam and Amba forests, located in Libo Kemkem District (Wereda) in the South Gondar Zone of the Amhara Regional State, northwestern Ethiopia located at around $12^{\circ} 04.351^{\prime}-12^{\circ} 10.926^{\prime} \mathrm{N}$ and $37^{\circ} 44.266^{\prime}-$ $37^{\circ} 50.057^{\prime} \mathrm{E}$. Tara-gedam forest ranges from 2062$2496 \mathrm{~m}$ a.s.l. and Amba from 2011-2541 m a.s.l. with the highest peak at Mt. Deboch. The climate data obtained from the National Meteorological Service Agency of Ethiopia shows that the mean annual maximum and minimum temperatures of the study area are $32.8^{\circ} \mathrm{C}$ and $8^{\circ} \mathrm{C}$, respectively. The District receives a uni-modal rainfall of approximately $1300 \mathrm{~mm}$ per year and about
95.1\% of the area is under moist weina dega (mid-highland) while the rest is under the wet Dega (highland) [16]. Medium and cold highland climatic features characterize the study area. The vegetation of the area belongs to the dry evergreen montane forest type consisting of forests, bushlands, shrublands and enrichment plantation interspersed with stands of natural vegetation [14]. Archival information [16] shows that forested land is about 4,429.5 hectares. Libo Kemkem District, in particular Tara-gedam, has several recreational sites. Mt. Kualla, along with diverse geographical features of the forest, Tara-gedam Monastery and many caves and forested churches are very useful for archaeological studies and for the tourism industry [17]. The 2007 census report of the Central Statistical Agency [18] of Ethiopia shows that Libo Kemkem District has an estimated population of 209,451 (106,564 males and 102,887 females). The inhabitants are mostly members of the Amhara ethnic community who speak the Amharic language with economies that are predominately based on rain-fed subsistence cultivation of crops mixed with livestock production [16]. There are 58 health services in the District [19]. Malaria, intestinal helminthiasis, and pneumonia were the top three human diseases and the major livestock ailments were pasteurllosis, anthrax, internal and external parasites, black leg, sheep pox, trypanosomiasis, respiratory tract infection, rabies and coccidiosis [20].

\section{Site selection methods and procedures}

Before starting the ethnobotanical study, contacts were made with various offices (District administration, tourism and culture, agriculture and rural development, traditional healers' association and health affairs) to seek permission to carry out the study by informing them about the aims and significance of the study. Letters authorizing the study were obtained from the relevant offices which were then presented to the concerned kebele (lowest administrative unit in Ethiopia) offices, forest scouts and informants in the study area. In this way, full legal procedures were followed and the informed consent of interested participants was obtained. Twelve rural villages, namely: Agamoch, Kidanemhret, Tibabosgie, Washa Indiras, Aguat Mafsesha, Mantogera, Abay, Kualla Yihuans, Yifag Akababi, Lomiye, Abuarra, Asiba Mariam and the town Addis Zemen were selected around the two forests. These villages are within the seven kebeles (Figure 1) selected for the study. Relative distance, community-forest interactions and altitudinal differences were the basic site selection criteria. Relative distance and community forest interaction were taken as criteria after collecting information from forest scouts, kebele administrative offices and inhabitants of the area during the reconnaissance survey in order to compare the indigenous 


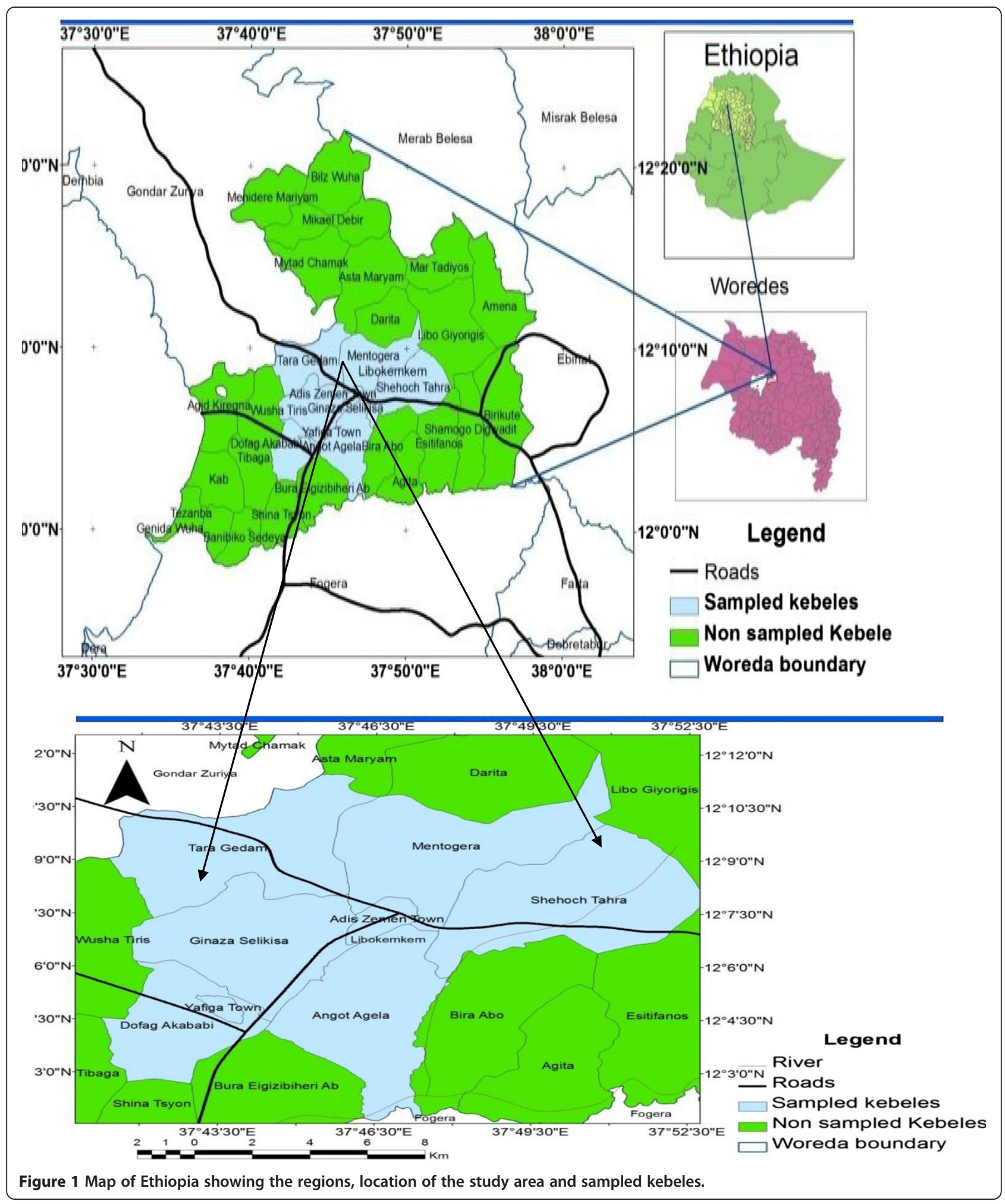

knowledge of the communities found nearest to the forest with those found relatively far away (reached after traveling for more than five kilometers). This was undertaken from November-June 2010.
Informant selection and approaches

One hundred five informants (85 males and 20 females) aged 19 to 84 were interviewed in this research. Among these, 45 (42 males and three females) were key 
informants and the rest 60 were general informants. Purposive and random sampling techniques were employed to select traditional herbalists and general informants respectively. The traditional association leaders, members of the tourism and culture office, elderly people and religious leaders helped to identify the key informants. In addition, the identified traditional practitioners and members who had earlier been treated by the healers also helped to identify other traditional experts. The general informants were randomly picked (from the list of inhabitants) during field and house visits (5-7 in each study site) by checking their names from the list of residents obtained from kebele offices. All interviews were administered after obtaining voluntary consent of each informant and assuring them that the data will be used only for academic purposes.

\section{Ways of data collection and type of data collected}

Ethnobotanical data were collected during three months from November to January 2010 by living in close contact with the community in the study area, following standard methods [2,4,21]. Accordingly, semi-structured interview, guided field walk, direct observation, market survey and focus group discussions with key informants and other knowledgeable community members were applied and their knowledge on medicinal plants gathered.

Interviews were held based on checklist of questions prepared before hand in English language and simultaneously translated into Amharic. Interviews focused to informant's demographic features including sex, age, marital status, occupation, religion, educational background, and duration of time an informant lived in the study area, and indigenous ecological knowledge (traditional ways of classifying vegetation, plants, landscapes and the soils in the area). The major part of the interviews were focused on the local names of medicinal plants used, their habits and habitats, plant part/s used, remedy preparation methods, materials used during preparation, condition of preparation, storage method, additives/ingredients used during preparation and administration, dosages administered, and route of administration. Likewise, side effect of the medicine (if any), use of antidotes for adverse effects, any taboos associated with medicinal plants, the season, month, dates and time of collection and preparation of plant medicines, and market value were also included. Further, the distribution (status) of medicinal plants, the interaction of healers with the District administration, threats and major problems, conservation methods, source of knowledge and ways of transfer and number of years of service as traditional healer were also the major interview points targeted, following the methods used by previous investigators $[2,4,22]$.

The semi-structured interviews held with informants usually started at their sitting places and further broadened into field walk with interviewed informants in order to see the plants mentioned in their habitats and voucher collections following Martin [4]. This activity further helped to record growth habits of medicinal plants. Focus group discussions were done with traditional medicinal plant association members, other herbalists, monks and general informants to obtain additional information and to check the reliability. Informants were contacted two to three times and responses of an informant in harmony with each other were taken as relevant and used for data analysis. At times, the preparation methods of the medicinal plants were said to be secret and were not included during discussion. Most field observations were conducted with a single informant in order to keep the knowledge top-secret as this was what the healers in particular preferred. Some of the traditional healers were genuine herbalists, well-known by the local community and owned traditional home pharmacies derived from plant remedies. They were asked to demonstrate their work at their homes and in the field, which was recorded in order to check the consistency in knowledge and practice on the preparation of remedies and their effectiveness. The patients encountered at healers' homes were also asked about the traditional plant medicines they have used and their effectiveness when applied by healers.

\section{Plant collection and identification}

Voucher specimens were collected for each plant species during guided field walk with the informants. At times, the field activities included taking notes on plants and the associated indigenous knowledge with preliminary identification of the plants to family and sometimes to species levels. Photographic records were also taken in the field to capture the field sites, plants and other useful memories. The specimens were dried, deep-frozen, and determinations were made at the National Herbarium (ETH), Addis Ababa University, using taxonomic keys and descriptions given in the relevant volumes of the Flora of Ethiopia and Eritrea [23-31] and by visual comparison with authenticated herbarium specimens. Finally, the accuracy of identifications was confirmed by a senior plant taxonomist and the voucher specimens with labels were deposited at the ETH.

\section{Data analysis}

The ethnobotanical data were analyzed using Microsoft Office Excel spreadsheet (2007) and SPSS version 20 software. The former was used to calculate sum, percentages, tabulate and draw graphs whereas the latter was used to generate results of descriptive statistics, and perform t-tests as well as draw graphs and charts. Ethnobotanical ranking and scoring methods such as preference and direct matrix rankings as well as pair-wise comparisons and informant consensuses were employed to distinguish priority species and to check consistency. 
Preference/priority ranking activities were employed on six most preferred and widely used medicinal plant species for the treatment of wound and the most threatened medicinal plants. Direct matrix ranking was employed for the six most widely utilized multi-purpose plant species and for the five factors considered most threatening to medicinal plants. Pair-wise comparison was made on six of the most preferred and commonly used medicinal plants against stomachache. To do this, the number of possible pairs was determined by applying the formula $n(n-1) / 2$, where $\mathrm{n}$ is the number of medicinal plant species being compared. For all the above ethnobotanical ranking and scoring techniques, the same seven key informants who had long time practical experience in traditional plant medicine preparation, administration and collection were engaged. The strength of knowledge of the key informants was evident to the first author who witnessed the clarity of explanations and accuracy of actions. The overall procedures for these activities were conducted following standard ethnobotany texts $[2,4,22]$. Informant consensus factor (ICF) for different ailment categories was calculated to test agreements of the informants on medicinal plant knowledge of each category by using the formula ICF $=$ Nur$\mathrm{Nu} / \mathrm{Nur}-1$ where, nur is the number of uses reported in each category and $\mathrm{Nu}$ is the number of species reported in each category [32].

\section{Ethical consideration}

All data collections were done with special care on the base of the cultural view of the local communities in the study area. Informants were also informed that the objectives of the research were not for commercial purposes but for academic reasons. Since, ethnomedicinal indigenous knowledge is only obtained from traditional specialists within the community so any value that will obtain as a result of the research will benefit the community. According to ethnobiology code of ethics indigenous knowledge should be protected and a part of the value generated should be transferred back to the authors of the knowledge. Finally, informants were accepted the idea and came to reach an agreement.

\section{Results}

Demographic features of the informants

Of the total informants, 46 were in the age group of 5185; 51 were illiterate and the greater proportion (88) belonged to the married category. Almost all informants (101) belonged to the Ethiopian Orthodox Church. Parallel to the population structure, there were more males than females who were willing to be included among informants as indicated by the demographic profile in Table 1.

Most informants (70) were farmers, 11 of them were house wives, seven were students and other groups were represented by fewer numbers. Of the total informants, 99 lived in the study area since birth and the rest have lived there from six to 20 years.

\section{Indigenous ecological knowledge of people in the study area}

The inhabitants of the study area are owners of rich ethnobotanical and ethnoecological knowledge as demonstrated by their wide array of knowledge on environmental matters. They classified the land forms; vegetation and soil based on knowledge surviving from ancestral practices (Table 2), now evident through their elaborate emic categorization systems.

\section{Medicinal plant diversity and distribution}

The study documented 163 species of medicinal plants belonging to 145 genera and 67 families. Three of the families had ten or more species each and the details are given in Table 3 and Table 4. The medicinal plant use reports showed that six species were cited by more than 20 informants each (Table 5). Twelve species were cited for the treatment of six and more ailments each (Table 6). Achyranthes aspera came out on the lists of both most effective and most cited medicinal plants and the details are tabulated (Table 4 and Table 5).

Among the reported medicinal plants of the area, some were also reported as wild edible plants (Table 4). Informants, during data collection, said that some of the species for example, the edible parts (fruits) of Rosa abyssinica are used to alleviate weakness or tension when eaten by children in the field. This is done without knowing the medicinal effects of the plants and those who eat it feel happy and accomplish their tasks effectively. Herbs accounted for $67(41.1 \%)$ species followed by shrubs (62, $38.0 \%)$, trees $(24,14.7 \%)$ and climbers $(10,6.1 \%)$. The medicinal plants occur in the wild, homegardens and in both premises. The forests, farmlands, margins, living plants on fences, roadsides, around homes, fallow lands and

Table 1 Demographic profile of the informants

\begin{tabular}{|c|c|c|c|c|c|c|c|c|c|c|}
\hline \multirow[t]{2}{*}{ Sex } & \multicolumn{3}{|c|}{ Age group (in yrs) } & \multicolumn{3}{|c|}{ Educational status } & \multicolumn{2}{|c|}{ Marital status } & \multicolumn{2}{|c|}{ Religious type } \\
\hline & $19-34$ & $35-50$ & $51-85$ & Illiterate & Religious education & Modern education & Single & Married & Orthodox & Muslim \\
\hline Male & 22 & 25 & 38 & 38 & 17 & 30 & 9 & 76 & 83 & 3 \\
\hline Female & 5 & 7 & 8 & 13 & 0 & 7 & 8 & 12 & 19 & 1 \\
\hline Total & 27 & 32 & 46 & 51 & 17 & 37 & 17 & 88 & 101 & 4 \\
\hline
\end{tabular}


Table 2 Emic categorization of landscape, soil and vegetation in the area

\begin{tabular}{|c|c|c|c|c|c|c|}
\hline \multicolumn{2}{|c|}{ Landscape (ethno-topographic) types } & \multicolumn{3}{|c|}{ Soil (ethnopedologic and scientific) types } & \multicolumn{2}{|c|}{ Vegetation (ethnofloristic) types } \\
\hline Amharic & English & Amharic & English & Scientific & Amharic & English \\
\hline WOTAGEBA & Up and down & KEYATIE & Red soil & Luvisols & KUTQUATO & Shrub \\
\hline TERRARAMA & Mountainous & WALKA & Black soil & Vertisols & GITOSH & Grass land \\
\hline MEDAMA/MESK & Plain & SERBOLA & Black \& white & Anthrosols & CHAKA & Forest \\
\hline SHELEQUAMA & Valley & $\mathrm{CHINCHA}$ & Brown & Leptosols & DENE & Plantation \\
\hline KOREBTA/GOBA & Outcrop land & BORENK & White & Cambisols & CHEBECHEB & Wetland (edaphic grassland) \\
\hline DAGET & Hilly & & & & & \\
\hline SINKURKUR & Stony place & & & & & \\
\hline
\end{tabular}

riversides are the habitats where the medicinal plants are found (Figure 2).

\section{Health disorders treated and ICF}

The analyses on application of plants showed that 115 (70.6\%) species in 103 genera and 54 families were listed as medicines for human ailments, 34 (20.9\%) species in 32 genera and 22 families for both human and livestock ailments and $14(8.6 \%)$ species in 14 genera and 11 families were reported as medicine for livestock ailments only. These medicinal plants were claimed to be of use in the treatment of about 60 types of human ailments only, 10 types of both human and livestock health disorders and nine types of livestock ailments only. For the most common ailment (wound), 42 medicinal plant species were reported (Table 7). The ailments were classified into 13 categories and ICF values were computed

Table 3 Plant families, number of medicinal plant species and proportions

\begin{tabular}{|c|c|c|c|}
\hline No & Family & $\begin{array}{l}\text { No of species } \\
\text { in each }\end{array}$ & $\%$ of total \\
\hline 1 & Asteraceae & 14 & 8.6 \\
\hline 2 & Fabaceae & 13 & 8.0 \\
\hline 3 & Solanaceae & 10 & 6.1 \\
\hline 4 & Euphorbiaceae & 8 & 4.9 \\
\hline 5 & Lamiaceae & 7 & 4.3 \\
\hline 6 & Malvaceae & 6 & 3.7 \\
\hline 7 & Apiaceae & 5 & 3.1 \\
\hline 8 & $\begin{array}{l}\text { Acanthaceae, Amarantaceae, } \\
\text { Asclepiadaceae, Cucurbitaceae, } \\
\text { Rubiaceae, Rutaceae (six families) }\end{array}$ & 4 & 2.5 \\
\hline 9 & $\begin{array}{l}\text { Convolvulaceae, Moraceae, } \\
\text { Rhamnaceae, Poaceae, Polygonaceae, } \\
\text { Oleaceae (six families) }\end{array}$ & 3 & 1.8 \\
\hline 10 & $\begin{array}{l}\text { Boraginaceae, Cuppressaceae, } \\
\text { Loganiaceae, Myrsinaceae, } \\
\text { Myrtaceae, Ranunculaceae, Rosaceae, } \\
\text { Scrophularaceae, Urticaceae, } \\
\text { Apocynaceae (ten families) }\end{array}$ & 2 & 1.2 \\
\hline 11 & Other 38 families & 1 & 0.6 \\
\hline
\end{tabular}

and livestock ailments had the highest ICF value of 0.84 and other disease categories had lower values (Table 8).

\section{Importance of the medicinal plants}

Some medicinal plants were rated as important and used frequently by many, appearing in many formulations. Preferences for six common medicinal plant species said to be used for the treatment of the common ailment (wound) showed Cordia africana in the first rank order followed by Sida rhombifolia (Table 9). The pair-wise comparison of medicinal plants used for the treatment of stomachache showed that Stephania abysinica was the most reported and ranked first, while Otostegia integrifolia was the least ranked plant species (Table 10). Matrix ranking of six popular multipurpose medicinal plants showed that Cordia africana was the most useful multipurpose medicinal plant that was ranked $1^{\text {st }}$ while Croton macrostachyus was the least ranked one (Table 11).

\section{Plant parts used and modes of remedy preparations}

Out of the total plant parts used for remedy preparation, leaves were the highest $(109,31.2 \%)$, followed by roots $(108,30.9 \%)$ and lower values for other parts (Table 12). Information about the preparation of each plant has been included in Table 4. The results also showed that the majorities of remedies (89\%) were prepared from single plant species and few (11\%) were prepared from combinations of more than two medicinal plant species. Simple modes of preparation of medicine including crushing (90.5\% informants), chewing, pounding, chopping and juice extraction were used (Table 13).

\section{Condition of preparation and storage of plant medicines}

The results of the analyses showed that $70.94 \%$ of the plant medicines were prepared from fresh plant parts, 9.69\% from dried and 19.37\% from both fresh and dried parts. Healers explained that some of the stored remedies were kept for about one year, from September to September of the next year and discarded on the Ethiopian New Year and replaced with new preparations. When a particular medicinal plant could not be 
Table 4 List of plant species used to treat human and livestock ailments: scientific names, family, vernacular name, growth forms (Gf), Ailments treated, Ailment type(At), parts used (Pu), condition of preparation (Cp), route of administration ( $\mathrm{Ra}$ ), method of preparation, habitat (Ha), distribution(Dn), collection number (Co.No.) in the environ of Tara-gedam and Amba forests

\begin{tabular}{|c|c|c|c|c|c|c|c|c|c|c|c|c|}
\hline $\begin{array}{l}\text { Scientific } \\
\text { Names }\end{array}$ & Family & $\begin{array}{l}\text { Vernacular name } \\
\text { (Amharic) }\end{array}$ & Gf & $\begin{array}{l}\text { Ailments } \\
\text { treated }\end{array}$ & At & $\mathrm{Pu}$ & $\mathrm{CP}$ & $\mathrm{Ra}$ & $\begin{array}{l}\text { Methods of } \\
\text { preparation }\end{array}$ & $\mathrm{Ha}$ & Dn & $\begin{array}{l}\text { Co. } \\
\text { No. }\end{array}$ \\
\hline $\begin{array}{l}\text { Acacia } \\
\text { abyssinica } \\
\text { Hochst. ex } \\
\text { Benth.* }\end{array}$ & Fabaceae & Girar & $\mathrm{T}$ & $\begin{array}{l}\text { Scorpion } \\
\text { poison }\end{array}$ & $\mathrm{Hu}$ & $B$ & $\mathrm{~F}$ & De & $\begin{array}{l}\text { Tie with } \\
\text { inside part }\end{array}$ & $F$ & Spr & GC097 \\
\hline $\begin{array}{l}\text { Acanthus } \\
\text { polystachius } \\
\text { Del. }\end{array}$ & Acanthaceae & Nech kusheshile & $S$ & Rabies & $\mathrm{Li}$ & $\mathrm{R}$ & $\mathrm{F}$ & $\mathrm{O}$ & $\begin{array}{l}\text { Pound and } \\
\text { give with } \\
\text { water }\end{array}$ & Fal & Spr & GC031 \\
\hline \multirow[t]{3}{*}{$\begin{array}{l}\text { Acanthus sennii } \\
\text { Chiov.* }\end{array}$} & Acanthaceae & Key kusheshilie & $S$ & Evil eye & $\mathrm{Hu}$ & $\mathrm{R}$ & FD & $\begin{array}{l}\mathrm{Na} \\
\mathrm{O} \& \\
\mathrm{De}\end{array}$ & $\begin{array}{l}\text { Sniff, drink } \\
\text { and fumigate } \\
\text { with } \\
\text { concoction }\end{array}$ & $\mathrm{F}$ & Wy & GC056 \\
\hline & & & & $\begin{array}{l}\text { Arthritis/ } \\
\text { rheumatism }\end{array}$ & $\mathrm{Hu}$ & $\mathrm{R}$ & $F$ & $\begin{array}{l}\text { De } \\
\& O\end{array}$ & $\begin{array}{l}\text { Crush \& tie or } \\
\text { drink with } \\
\text { honey }\end{array}$ & Bo & & \\
\hline & & & & Tape worm & $\mathrm{Hu}$ & $\mathrm{R}$ & $\mathrm{F}$ & $\mathrm{O}$ & $\begin{array}{l}\text { Pound, } \\
\text { immerse in } \\
\text { water then } \\
\text { drink the } \\
\text { juice }\end{array}$ & & & \\
\hline \multirow[t]{9}{*}{$\begin{array}{l}\text { Achyranthes } \\
\text { aspera L. }\end{array}$} & Amaranthaceae & Telenj & $\mathrm{H}$ & Eye problem & $\mathrm{Hu}$ & $L$ & $\mathrm{~F}$ & $O p$ & $\begin{array}{l}\text { Pound, } \\
\text { immerse to } \\
\text { water, } \\
\text { squeeze and } \\
\text { insert with } \\
\text { cotton }\end{array}$ & & Wy & GC025 \\
\hline & & & & Wound & $\mathrm{Hu}$ & $L$ & FD & De & $\begin{array}{l}\text { Crush, } \\
\text { powder and } \\
\text { tie }\end{array}$ & & & \\
\hline & & & & Wound & $\mathrm{Hu}$ & $L$ & $\mathrm{~F}$ & De & Crush and tie & & & \\
\hline & & & & $\begin{array}{l}\text { Excessive } \\
\text { menstruation }\end{array}$ & $\mathrm{Hu}$ & $\mathrm{R}$ & $\mathrm{F}$ & $\mathrm{O}$ & $\begin{array}{l}\text { Crush, insert } \\
\text { in water then } \\
\text { drink juice }\end{array}$ & & & \\
\hline & & & & Tonsillitis & $\mathrm{Hu}$ & $L$ & $\mathrm{~F}$ & De & Crush and tie & & & \\
\hline & & & & Bleeding & $\mathrm{Li}$ & $\mathrm{R}$ & $\mathrm{F}$ & De & Crush and tie & & & \\
\hline & & & & Bone fracture & $B$ & $\mathrm{R}$ & FD & De & $\begin{array}{l}\text { Tie the } \\
\text { concoction }\end{array}$ & & & \\
\hline & & & & Bleeding & $\mathrm{Hu}$ & $\mathrm{R}$ & $\mathrm{FD}$ & De & $\begin{array}{l}\text { Tie the } \\
\text { concoction }\end{array}$ & & & \\
\hline & & & & Tape worm & $\mathrm{Hu}$ & $\mathrm{R}$ & $\mathrm{F}$ & $\mathrm{O}$ & $\begin{array}{l}\text { Crush, insert } \\
\text { in water then } \\
\text { drink }\end{array}$ & & & \\
\hline $\begin{array}{l}\text { Acmella } \\
\text { caulirhiza Del. }\end{array}$ & Asteraceae & Kutchamelk & $\mathrm{H}$ & Swelling & $\mathrm{Hu}$ & $L$ & FD & De & $\begin{array}{l}\text { Crush and } \\
\text { powder then } \\
\text { tie with } \\
\text { honey/better }\end{array}$ & $\mathrm{Hg}$ & $\mathrm{Pa}$ & GC134 \\
\hline \multirow[t]{2}{*}{$\begin{array}{l}\text { Acokanthera } \\
\text { schimperi (A. } \\
\text { DC.) Schweinf. }\end{array}$} & Apocynaceae & Merz/Mirez & $S$ & Spider poison & $\mathrm{Hu}$ & $\mathrm{L}$ & $D$ & De & $\begin{array}{l}\text { Crush and } \\
\text { powder then } \\
\text { cream with } \\
\text { butter }\end{array}$ & Bo & $\mathrm{Rr}$ & GC047 \\
\hline & & & & Hepatitis & $\mathrm{Hu}$ & Ap & $\mathrm{D}$ & $\begin{array}{l}\mathrm{Na} \\
\mathrm{O} \& \\
\mathrm{De}\end{array}$ & $\begin{array}{l}\text { Crush, dry } \\
\text { then fumigate }\end{array}$ & & & \\
\hline
\end{tabular}


Table 4 List of plant species used to treat human and livestock ailments: scientific names, family, vernacular name, growth forms (Gf), Ailments treated, Ailment type(At), parts used (Pu), condition of preparation (Cp), route of administration (Ra), method of preparation, habitat (Ha), distribution(Dn), collection number (Co.No.) in the environ of Tara-gedam and Amba forests (Continued)

\begin{tabular}{|c|c|c|c|c|c|c|c|c|c|c|c|c|}
\hline \multirow[t]{2}{*}{$\begin{array}{l}\text { Adiantum } \\
\text { capillus-veneris } \\
\text { L. }\end{array}$} & \multirow[t]{2}{*}{ Adiantaceae } & \multirow[t]{2}{*}{ Joroasfit } & \multirow[t]{2}{*}{$\mathrm{H}$} & Anthrax & $\mathrm{Hu}$ & $\mathrm{R}$ & $F$ & $\mathrm{O}$ & $\begin{array}{l}\text { Crush, insert } \\
\text { in water then } \\
\text { drink the } \\
\text { juice }\end{array}$ & \multirow[t]{2}{*}{$\mathrm{F}$} & \multirow[t]{2}{*}{ Spr } & \multirow[t]{2}{*}{ GC027 } \\
\hline & & & & Ear wound & $\mathrm{Hu}$ & St & FD & De & $\begin{array}{l}\text { Insert into } \\
\text { new jewelry } \\
\text { hole }\end{array}$ & & & \\
\hline \multirow[t]{5}{*}{$\begin{array}{l}\text { Allium sativum } \\
\mathrm{L} \text {. }\end{array}$} & \multirow[t]{5}{*}{ Alliaceae } & \multirow[t]{5}{*}{ Nech shinkurt } & \multirow[t]{5}{*}{$\mathrm{H}$} & Evil eye & $\mathrm{Hu}$ & $\mathrm{Bu}$ & $\mathrm{F}$ & $\begin{array}{l}\mathrm{Na} \\
\mathrm{O} \& \\
\mathrm{De}\end{array}$ & $\begin{array}{l}\text { Sniff, drink } \\
\text { and fumigate } \\
\text { with } \\
\text { concoction }\end{array}$ & \multirow[t]{5}{*}{$\mathrm{Hg}$} & \multirow[t]{5}{*}{ Spr } & \multirow[t]{5}{*}{ GC011 } \\
\hline & & & & Malaria & $\mathrm{Hu}$ & $\mathrm{Bu}$ & $\mathrm{F}$ & $\mathrm{O}$ & $\begin{array}{l}\text { Crush and } \\
\text { drink with } \\
\text { honey or } \\
\text { smash in } \\
\text { water then } \\
\text { drink }\end{array}$ & & & \\
\hline & & & & Influenza virus & $\mathrm{Hu}$ & $\mathrm{Bu}$ & $F$ & $\mathrm{O}$ & $\begin{array}{l}\text { Crush and } \\
\text { drink with } \\
\text { water }\end{array}$ & & & \\
\hline & & & & Febrile illness & $\mathrm{Hu}$ & $\mathrm{Bu}$ & $\mathrm{F}$ & $\mathrm{O}$ & $\begin{array}{l}\text { Crush then } \\
\text { fumigate or } \\
\text { drink the } \\
\text { concoction }\end{array}$ & & & \\
\hline & & & & Pneumonia & $\mathrm{Hu}$ & $\mathrm{Bu}$ & $F$ & $\mathrm{O}$ & $\begin{array}{l}\text { Chop and eat } \\
\text { with honey }\end{array}$ & & & \\
\hline \multirow[t]{2}{*}{$\begin{array}{l}\text { Aloe } \\
\text { macrocarpa } \\
\text { Tod. }\end{array}$} & \multirow[t]{2}{*}{ Aloaceae } & \multirow[t]{2}{*}{ Eret } & \multirow[t]{2}{*}{$\mathrm{H}$} & Impotency & $\mathrm{Hu}$ & $\mathrm{R}$ & $F$ & De & $\begin{array}{l}\text { Crush and } \\
\text { powder, then } \\
\text { cream with } \\
\text { butter }\end{array}$ & \multirow[t]{2}{*}{ Fwl } & \multirow[t]{2}{*}{$\operatorname{Rr}$} & \multirow[t]{2}{*}{ GC034 } \\
\hline & & & & Wound & B & $L x$ & $\mathrm{~F}$ & De & Creamed & & & \\
\hline $\begin{array}{l}\text { Alternanthera } \\
\text { pungens Kunth }\end{array}$ & Amaranthaceae & Midir akef & $\mathrm{H}$ & $\begin{array}{l}\text { Babies } \\
\text { diseases }\end{array}$ & $\mathrm{Hu}$ & $\mathrm{L}$ & $F$ & De & $\begin{array}{l}\text { Rub, squeeze } \\
\text { then cream }\end{array}$ & Bo & $\mathrm{Rr}$ & GC146 \\
\hline $\begin{array}{l}\text { Alysicarpus } \\
\text { quartinianus A. } \\
\text { Rich. }\end{array}$ & Fabaceae & -————— & $\mathrm{H}$ & Ascaris & $\mathrm{Hu}$ & $\mathrm{R}$ & $\mathrm{F}$ & $\mathrm{O}$ & $\begin{array}{l}\text { Crush then } \\
\text { drink with } \\
\text { milk }\end{array}$ & Fwl & $\operatorname{Rr}$ & GC142 \\
\hline $\begin{array}{l}\text { Argemone } \\
\text { mexicana L. }\end{array}$ & Papaveraceae & Yahya eshoh & $\mathrm{H}$ & Rabies & $\mathrm{Li}$ & $\mathrm{R}$ & $F$ & $\mathrm{O}$ & $\begin{array}{l}\text { Crush then } \\
\text { give with } \\
\text { water }\end{array}$ & Rs & Wy & GC058 \\
\hline $\begin{array}{l}\text { Artemisia afra } \\
\text { Jack. ex Willd. }\end{array}$ & Asteraceae & Chikugn & $\mathrm{H}$ & Evil eye & $\mathrm{Hu}$ & Ap & FD & $\begin{array}{l}\mathrm{Na} \\
\mathrm{O} \& \\
\mathrm{De}\end{array}$ & $\begin{array}{l}\text { Sniff } \\
\text { unprocessed } \\
\text { and powder } \\
\text { then fumigate } \\
\text { and drink } \\
\text { concoction }\end{array}$ & $\mathrm{Hg}$ & $\mathrm{Rr}$ & GC168 \\
\hline \multirow[t]{3}{*}{$\begin{array}{l}\text { Asparagus } \\
\text { africanus Lam. }\end{array}$} & \multirow[t]{3}{*}{ Asparagaceae } & \multirow[t]{3}{*}{ Yesiet kest } & \multirow[t]{3}{*}{$S$} & $\begin{array}{l}\text { Impotency, } \\
\text { gonnoria,\& } \\
\text { syphilis }\end{array}$ & $\mathrm{Hu}$ & $\mathrm{R}$ & DF & $\mathrm{O}$ & $\begin{array}{l}\text { Crush, } \\
\text { infusion with } \\
\text { honey then } \\
\text { drink the } \\
\text { juice }\end{array}$ & Fal & Spr & GC151 \\
\hline & & & & Itchiness & $\mathrm{Hu}$ & $\mathrm{R}$ & DF & De & $\begin{array}{l}\text { Crush, } \\
\text { powder then } \\
\text { cream with } \\
\text { butter }\end{array}$ & & & \\
\hline & & & & $\begin{array}{l}\text { Excessive } \\
\text { menstruation }\end{array}$ & $\mathrm{Hu}$ & $\mathrm{R}$ & $F$ & $\mathrm{O}$ & $\begin{array}{l}\text { Chew and } \\
\text { swallow the } \\
\text { juice }\end{array}$ & & & \\
\hline
\end{tabular}


Table 4 List of plant species used to treat human and livestock ailments: scientific names, family, vernacular name, growth forms (Gf), Ailments treated, Ailment type(At), parts used (Pu), condition of preparation (Cp), route of administration (Ra), method of preparation, habitat (Ha), distribution(Dn), collection number (Co.No.) in the environ of Tara-gedam and Amba forests (Continued)

\begin{tabular}{|c|c|c|c|c|c|c|c|c|c|c|c|c|}
\hline & & & & Evil eye & $\mathrm{Hu}$ & $\mathrm{R}$ & DF & $\begin{array}{l}\mathrm{Na} \\
\mathrm{O} \& \\
\mathrm{De}\end{array}$ & $\begin{array}{l}\text { Sniff, drink } \\
\text { and fumigate } \\
\text { concoction }\end{array}$ & & & \\
\hline $\begin{array}{l}\text { Astragalus } \\
\text { atropilosus } \\
\text { (Hochst.) Bunge }\end{array}$ & Fabaceae & -二-二-- - - & $\mathrm{H}$ & Itchiness & $\mathrm{Hu}$ & Ap & $\mathrm{D}$ & De & $\begin{array}{l}\text { Dry, burn } \\
\text { then cream } \\
\text { ash with } \\
\text { butter }\end{array}$ & Fal & Spr & GC152 \\
\hline $\begin{array}{l}\text { Bersama } \\
\text { abyssinica } \\
\text { Fresen. }\end{array}$ & Melianthaceae & Azamir & S & Ascaris & $\mathrm{Hu}$ & $L$ & FD & $\mathrm{O}$ & $\begin{array}{l}\text { Crush and } \\
\text { powder, boil } \\
\text { with tea then } \\
\text { drink juice }\end{array}$ & Aw & Spr & GC107 \\
\hline $\begin{array}{l}\text { Bidens } \\
\text { macroptera } \\
\text { (Sch Bip.) ex } \\
\text { Chiov. Mesfin }\end{array}$ & Asteraceae & Adey Abeba & $\mathrm{H}$ & Brain cancer & $\mathrm{Hu}$ & $\mathrm{Fl}$ & $\mathrm{D}$ & $\mathrm{Na}$ & Powdered & Fal & Wy & GC143 \\
\hline $\begin{array}{l}\text { Brassica } \\
\text { carinata A. Br. }\end{array}$ & Brassicaceae & Gomen & $\mathrm{H}$ & $\begin{array}{l}\text { Stomachache } \\
\text { \& Anthrax }\end{array}$ & $B$ & Sd & $\mathrm{D}$ & $\mathrm{O}$ & $\begin{array}{l}\text { Grind and } \\
\text { drink with } \\
\text { water }\end{array}$ & $\mathrm{Hg}$ & Wy & GC176 \\
\hline $\begin{array}{l}\text { Bridelia } \\
\text { micrantha } \\
\text { (Hochst.) Brain. }\end{array}$ & Euphorbiaceae & Yenebr tifir & $\mathrm{T}$ & $\begin{array}{l}\text { Expel } \\
\text { placenta }\end{array}$ & $\mathrm{Li}$ & $B$ & $\mathrm{~F}$ & $\mathrm{O}$ & $\begin{array}{l}\text { Crush then } \\
\text { give with } \\
\text { water }\end{array}$ & Rs & Rare & GC089 \\
\hline \multirow[t]{2}{*}{$\begin{array}{l}\text { Brucea } \\
\text { antidysenterica } \\
\text { Swiss Chard. }\end{array}$} & Simaroubaceae & Waynos/yedaga abalo & $H$ & $\begin{array}{l}\text { Wound \& } \\
\text { Scabies }\end{array}$ & $\mathrm{Hu}$ & $L$ & D & De & $\begin{array}{l}\text { Crush, mixed } \\
\text { with butter } \\
\text { then cream }\end{array}$ & Fal & Spr & GC086 \\
\hline & & & & Skin rash & $\mathrm{Li}$ & $L$ & $\mathrm{D}$ & De & $\begin{array}{l}\text { Crush, mix } \\
\text { with butter } \\
\text { then cream }\end{array}$ & & & \\
\hline \multirow[t]{4}{*}{$\begin{array}{l}\text { Buddleja } \\
\text { polystachya } \\
\text { Fresen. }\end{array}$} & Loganiaceae & Anfar & $S$ & Tonsillitis & $\mathrm{Hu}$ & Sh & $F$ & De & $\begin{array}{l}\text { Tie and } \\
\text { cream } \\
\text { concoction }\end{array}$ & $\mathrm{F}$ & Spr & GC062 \\
\hline & & & & $\begin{array}{l}\text { Intestinal } \\
\text { parasite }\end{array}$ & $\mathrm{Hu}$ & $\mathrm{L}$ & D & $\mathrm{O}$ & $\begin{array}{l}\text { Crush and } \\
\text { powder, } \\
\text { immerse in } \\
\text { TEJ then drink } \\
\text { the juice }\end{array}$ & & & \\
\hline & & & & $\begin{array}{l}\text { Excessive } \\
\text { menstruation }\end{array}$ & $\mathrm{Hu}$ & $\mathrm{L}$ & $F$ & Va & $\begin{array}{l}\text { Make soft by } \\
\text { rubbing, and } \\
\text { insert with } \\
\text { new cloth } \\
\text { until bleeding } \\
\text { stops }\end{array}$ & & & \\
\hline & & & & Wound & $\mathrm{Hu}$ & Sh & $F$ & De & Crush and tie & & & \\
\hline \multirow{2}{*}{$\begin{array}{l}\text { Calotropis } \\
\text { procera (Ait.) } \\
\text { Ait.f. }\end{array}$} & Asclepiadaceae & Tobia & S & Hemorrhoid & $\mathrm{Hu}$ & $L x$ & $\mathrm{~F}$ & De & $\begin{array}{l}\text { Cream } \\
\text { concoction }\end{array}$ & Rs & Spr & GC035 \\
\hline & & & & $\begin{array}{l}\text { Expel spine in } \\
\text { wound }\end{array}$ & $\mathrm{Hu}$ & $L x$ & $\mathrm{~F}$ & De & $\begin{array}{l}\text { Cream on } \\
\text { point }\end{array}$ & & & \\
\hline \multirow[t]{3}{*}{$\begin{array}{l}\text { Calpurnia aurea } \\
\text { (Ait.) Benth. }\end{array}$} & Fabaceae & Zikita & S & $\begin{array}{l}\text { External } \\
\text { parasites }\end{array}$ & $\mathrm{Li}$ & $\mathrm{L}$ & $\mathrm{F}$ & De & $\begin{array}{l}\text { Crush, then } \\
\text { wash with } \\
\text { water }\end{array}$ & Bo & Spr & GC020 \\
\hline & & & & $\begin{array}{l}\text { Diarrhea \& } \\
\text { Bilharziasis }\end{array}$ & $\mathrm{Hu}$ & Sd & D & $\mathrm{O}$ & $\begin{array}{l}\text { Grind and eat } \\
\text { after } \\
\text { pounding } \\
\text { with honey }\end{array}$ & & & \\
\hline & & & & $\begin{array}{l}\text { Bloody } \\
\text { diarrhea }\end{array}$ & B & $\mathrm{R}$ & $\mathrm{F}$ & $\mathrm{O}$ & $\begin{array}{l}\text { Crush then } \\
\text { drink with } \\
\text { water }\end{array}$ & & & \\
\hline
\end{tabular}


Table 4 List of plant species used to treat human and livestock ailments: scientific names, family, vernacular name, growth forms (Gf), Ailments treated, Ailment type(At), parts used (Pu), condition of preparation (Cp), route of administration (Ra), method of preparation, habitat (Ha), distribution(Dn), collection number (Co.No.) in the environ of Tara-gedam and Amba forests (Continued)

\begin{tabular}{|c|c|c|c|c|c|c|c|c|c|c|c|c|}
\hline & & & & Erthroblastosis & $\mathrm{Hu}$ & $\mathrm{Sd}$ & $\mathrm{D}$ & $\begin{array}{l}\text { De } \\
\& O\end{array}$ & $\begin{array}{l}\text { Grind and } \\
\text { drink with } \\
\text { honey or tie } \\
\text { powder/ } \\
\text { concoction } \\
\text { on neck }\end{array}$ & & & \\
\hline & & & & $\begin{array}{l}\text { Expel foreign } \\
\text { things from } \\
\text { eye }\end{array}$ & $\mathrm{Hu}$ & L & $\mathrm{F}$ & Et & $\begin{array}{l}\text { Crush } \\
\text { mixture, } \\
\text { squeeze then } \\
\text { insert with } \\
\text { cotton wool }\end{array}$ & & & \\
\hline & & & & $\begin{array}{l}\text { Prolonged } \\
\text { embryo in } \\
\text { uterus }\end{array}$ & $\mathrm{Hu}$ & $R$ & DF & De & $\begin{array}{l}\text { Tie } \\
\text { concoction } \\
\text { on spinal } \\
\text { column }\end{array}$ & & & \\
\hline \multirow[t]{2}{*}{$\begin{array}{l}\text { Capparis } \\
\text { tomentosa Lam. } \\
*\end{array}$} & \multirow[t]{2}{*}{ Capparidaceae } & \multirow[t]{2}{*}{ Gimero } & \multirow[t]{2}{*}{ S } & Evil eye & $\mathrm{Hu}$ & $R$ & DF & $\begin{array}{l}\mathrm{Na} \\
\mathrm{O} \& \\
\mathrm{De}\end{array}$ & $\begin{array}{l}\text { Sniff, drink } \\
\text { and fumigate } \\
\text { concoction }\end{array}$ & $\mathrm{F}$ & Wy & GC023 \\
\hline & & & & Epidemic & $\mathrm{Hu}$ & R & D & De & $\begin{array}{l}\text { Burn the } \\
\text { concoction } \\
\text { and fumigate }\end{array}$ & & & \\
\hline $\begin{array}{l}\text { Capsicum } \\
\text { annuum L. }\end{array}$ & Solanaceae & Karia/keto & $\mathrm{H}$ & Malaria & $\mathrm{Hu}$ & $\mathrm{Fr}$ & $\mathrm{F}$ & O & $\begin{array}{l}\text { Crush and } \\
\text { drink with } \\
\text { honey or } \\
\text { smash in } \\
\text { water then } \\
\text { drink }\end{array}$ & $\mathrm{Hg}$ & Wy & GC026 \\
\hline \multirow[t]{2}{*}{$\begin{array}{l}\text { Carica papaya } \\
\text { L. }\end{array}$} & \multirow[t]{2}{*}{ Caricaceae } & \multirow[t]{2}{*}{ Papya } & \multirow[t]{2}{*}{$\mathrm{T}$} & Malaria & $\mathrm{Hu}$ & L & $\mathrm{F}$ & O & $\begin{array}{l}\text { Crush and } \\
\text { drink with } \\
\text { milk }\end{array}$ & $\mathrm{Hg}$ & Spr & GC098 \\
\hline & & & & Cough & $\mathrm{Hu}$ & $R$ & $\mathrm{~F}$ & $\mathrm{O}$ & $\begin{array}{l}\text { Crush and } \\
\text { boil with tea } \\
\text { then drink } \\
\text { juice }\end{array}$ & & & \\
\hline \multirow[t]{3}{*}{$\begin{array}{l}\text { Carissa } \\
\text { spinarum L. * }\end{array}$} & \multirow[t]{3}{*}{ Apocynaceae } & \multirow[t]{3}{*}{ Agam } & \multirow[t]{3}{*}{ S } & Evil eye & $\mathrm{Hu}$ & $\mathrm{R}$ & FDD & $\begin{array}{l}\mathrm{Na}, \\
\mathrm{O} \& \\
\mathrm{De}\end{array}$ & $\begin{array}{l}\text { Sniff, drink } \\
\text { and fumigate } \\
\text { concoction }\end{array}$ & \multirow[t]{3}{*}{$\mathrm{F}$} & \multirow[t]{3}{*}{ Wy } & \multirow[t]{3}{*}{ GC021 } \\
\hline & & & & Epidemic & $\mathrm{Hu}$ & $\mathrm{R}$ & D & $\begin{array}{l}\mathrm{Na} \\
\mathrm{O} \& \\
\mathrm{De}\end{array}$ & $\begin{array}{l}\text { Burn the } \\
\text { mixture and } \\
\text { fumigate }\end{array}$ & & & \\
\hline & & & & $\begin{array}{l}\text { Brain tension/ } \\
\text { stress }\end{array}$ & $\mathrm{Hu}$ & $R$ & $\mathrm{D}$ & $\mathrm{Na}$ & $\begin{array}{l}\text { Crush then } \\
\text { fumigate }\end{array}$ & & & \\
\hline $\begin{array}{l}\text { Cayratia gracilis } \\
\text { (Guill.\&Perr.) } \\
\text { Suesseng }\end{array}$ & Vitaceae & Aserkush & $\mathrm{Cl}$ & Hemorrhoid & $\mathrm{Hu}$ & $R$ & $\mathrm{~F}$ & De & $\begin{array}{l}\text { Cream } \\
\text { concoction }\end{array}$ & $\mathrm{Fwl}$ & Spr & GC052 \\
\hline $\begin{array}{l}\text { Celosia trigyna } \\
\mathrm{L} \text {. }\end{array}$ & Amaranthaceae & Lemlemcho & $\mathrm{H}$ & Tape worm & $\mathrm{Hu}$ & $\mathrm{Sd}$ & D & $\mathrm{O}$ & $\begin{array}{l}\text { Grind and } \\
\text { drink with } \\
\text { water }\end{array}$ & $\mathrm{Hg}$ & Spr & GC132 \\
\hline \multirow[t]{2}{*}{$\begin{array}{l}\text { Chenopodium } \\
\text { murale L. }\end{array}$} & \multirow[t]{2}{*}{ Chenopodiaceae } & \multirow[t]{2}{*}{ Amedmado } & \multirow[t]{2}{*}{$\mathrm{H}$} & Wound & $\mathrm{Hu}$ & $L$ & DF & De & $\begin{array}{l}\text { Crush then } \\
\text { cream with } \\
\text { butter }\end{array}$ & \multirow[t]{2}{*}{$\mathrm{Hg}$} & \multirow[t]{2}{*}{$\mathrm{Rr}$} & GC136 \\
\hline & & & & Ear problem & $\mathrm{Hu}$ & L & $\mathrm{F}$ & De & $\begin{array}{l}\text { Concoction } \\
\text { inserted to } \\
\text { ear tube }\end{array}$ & & & \\
\hline
\end{tabular}


Table 4 List of plant species used to treat human and livestock ailments: scientific names, family, vernacular name, growth forms (Gf), Ailments treated, Ailment type(At), parts used (Pu), condition of preparation (Cp), route of administration (Ra), method of preparation, habitat (Ha), distribution(Dn), collection number (Co.No.) in the environ of Tara-gedam and Amba forests (Continued)

\begin{tabular}{|c|c|c|c|c|c|c|c|c|c|c|c|c|}
\hline $\begin{array}{l}\text { Cicer arietinum } \\
\mathrm{L} \text {. }\end{array}$ & Fabaceae & Shinbira & $\mathrm{H}$ & Malaria & $\mathrm{Hu}$ & $\mathrm{Sd}$ & D & $\mathrm{O}$ & $\begin{array}{l}\text { Germinate } \\
\text { then eat with } \\
\text { bulb of Allium } \\
\text { sativum }\end{array}$ & Bo & Wy & GC115 \\
\hline \multirow[t]{3}{*}{$\begin{array}{l}\text { Cirsium } \\
\text { englerianum } \mathrm{O} \text {. } \\
\text { Hoffm. }\end{array}$} & Asteraceae & Yahyakusheshilie & $\mathrm{H}$ & $\begin{array}{l}\text { Beating with } \\
\text { stick }\end{array}$ & $\mathrm{Li}$ & St & $\mathrm{F}$ & $\mathrm{O}$ & $\begin{array}{l}\text { Crush, } \\
\text { immerse in } \\
\text { water then } \\
\text { drink juice }\end{array}$ & $\mathrm{F}$ & Spr & GC050 \\
\hline & & & & Scabies & $\mathrm{Hu}$ & Sh & $\mathrm{F}$ & $\mathrm{De}$ & $\begin{array}{l}\text { Crush, roast } \\
\text { then cream }\end{array}$ & & & \\
\hline & & & & Influenza virus & $\mathrm{Hu}$ & $\mathrm{Fr}$ & $\mathrm{F}$ & $\mathrm{O}$ & $\begin{array}{l}\text { Crush and } \\
\text { drink with } \\
\text { water }\end{array}$ & & & \\
\hline $\begin{array}{l}\text { Citrus } \\
\text { aurantifolia } \\
\text { Burn. f. }\end{array}$ & Rutaceae & Lomy & $S$ & Wound & $\mathrm{Hu}$ & $\mathrm{Fr}$ & $\mathrm{F}$ & De & $\begin{array}{l}\text { Cream } \\
\text { concoction }\end{array}$ & $\mathrm{Hg}$ & Spr & GC169 \\
\hline $\begin{array}{l}\text { Citrus } \\
\text { aurantium L. }\end{array}$ & Rutaceae & komtatie & S & Hypertension & $\mathrm{Hu}$ & $\mathrm{Fl}$ & $\mathrm{F}$ & $\mathrm{O}$ & $\begin{array}{l}\text { Drink the } \\
\text { juice }\end{array}$ & $\mathrm{Hg}$ & $\operatorname{Rr}$ & GC138 \\
\hline $\begin{array}{l}\text { Clausena } \\
\text { anisata (Willd.) } \\
\text { Benth. }\end{array}$ & Rutaceae & Limich & $S$ & Evil eye & $\mathrm{Hu}$ & $\mathrm{R}$ & D & $\begin{array}{l}\mathrm{Na} \\
\mathrm{O} \& \\
\mathrm{De}\end{array}$ & $\begin{array}{l}\text { Sniff, drink } \\
\text { and fumigate } \\
\text { with } \\
\text { concoction }\end{array}$ & $\mathrm{F}$ & Spr & GC178 \\
\hline \multirow[t]{3}{*}{$\begin{array}{l}\text { Clematis } \\
\text { simensis Fresen. }\end{array}$} & Ranunculaceae & Azo areg & $\mathrm{Cl}$ & Hemorrhoid & $\mathrm{Hu}$ & $L$ & $\mathrm{~F}$ & De & $\begin{array}{l}\text { Crush then } \\
\text { tied }\end{array}$ & $\mathrm{F}$ & Spr & GC043 \\
\hline & & & & Wound & B & $L$ & $\mathrm{~F}$ & $\mathrm{De}$ & $\begin{array}{l}\text { Crush then } \\
\text { tied }\end{array}$ & & & \\
\hline & & & & Cancer & $\mathrm{Hu}$ & $L$ & $F$ & De & $\begin{array}{l}\text { Crush and } \\
\text { powder then } \\
\text { cream }\end{array}$ & & & \\
\hline $\begin{array}{l}\text { Clerodendrum } \\
\text { myricoides } \\
\text { (Hochst.) Vatke }\end{array}$ & Lamiaceae & Misroch & S & $\begin{array}{l}\text { Evil eye \& evil } \\
\text { sprit }\end{array}$ & $\mathrm{Hu}$ & $\begin{array}{l}L, R \\
\& S d\end{array}$ & FD & $\begin{array}{l}\text { De } \\
\& O\end{array}$ & $\begin{array}{l}\text { Crush, } \\
\text { powder then } \\
\text { tie on the } \\
\text { neck or take } \\
\text { with tooth }\end{array}$ & $\mathrm{F}$ & Spr & GC016 \\
\hline \multirow[t]{4}{*}{$\begin{array}{l}\text { Clutia } \\
\text { lanceolata } \\
\text { Forssk. }\end{array}$} & Euphorbiaceae & Fiyelefej & S & Diarrhea & $\mathrm{Hu}$ & $\mathrm{R}$ & $\mathrm{F}$ & $\mathrm{De}$ & $\begin{array}{l}\text { Crush then tie } \\
\text { on neck } \\
\text { region }\end{array}$ & FWl & Wy & GC135 \\
\hline & & & & Bone fracture & $\mathrm{Hu}$ & $\mathrm{R}$ & $\mathrm{F}$ & De & Crush and tie & & & \\
\hline & & & & $\begin{array}{l}\text { Beating with } \\
\text { stick }\end{array}$ & $\mathrm{Li}$ & $\mathrm{L}$ & $F$ & $\mathrm{O}$ & $\begin{array}{l}\text { Crush and } \\
\text { give with } \\
\text { water }\end{array}$ & & & \\
\hline & & & & $\begin{array}{l}\text { Expel ear } \\
\text { mites }\end{array}$ & $\mathrm{Hu}$ & $\mathrm{Fr}$ & $F$ & Et & $\begin{array}{l}\text { Grind, insert } \\
\text { into ear tube } \\
\text { until it expels } \\
\text { mites }\end{array}$ & & & \\
\hline \multirow[t]{2}{*}{$\begin{array}{l}\text { Coffea arabica } \\
\text { L. }\end{array}$} & Rubiaceae & Bunna & $S$ & $\begin{array}{l}\text { Common } \\
\text { cold }\end{array}$ & $\mathrm{Hu}$ & $L$ & $\mathrm{~F}$ & $\mathrm{O}$ & $\begin{array}{l}\text { Boil, decant } \\
\text { then drink } \\
\text { the juice }\end{array}$ & $\mathrm{Hg}$ & Spr & GC161 \\
\hline & & & & Diarrhea & $\mathrm{Hu}$ & $\mathrm{Fr}$ & $\mathrm{F}$ & $\mathrm{O}$ & $\begin{array}{l}\text { Grind and eat } \\
\text { with honey }\end{array}$ & & & \\
\hline \multirow{2}{*}{$\begin{array}{l}\text { Commelina } \\
\text { latifolia Hochst. } \\
\text { ex A Rich. }\end{array}$} & Commelinaceae & Yewuha enkur & $\mathrm{H}$ & Wound & $\mathrm{Hu}$ & $L$ & $\mathrm{~F}$ & De & Crush and tie & Ris & Spr & GC116 \\
\hline & & & & Taenia scaplis & $\mathrm{Hu}$ & $L$ & D & De & $\begin{array}{l}\text { Crush and } \\
\text { powder then } \\
\text { cream with } \\
\text { butter }\end{array}$ & & & \\
\hline
\end{tabular}


Table 4 List of plant species used to treat human and livestock ailments: scientific names, family, vernacular name, growth forms (Gf), Ailments treated, Ailment type(At), parts used (Pu), condition of preparation (Cp), route of administration (Ra), method of preparation, habitat (Ha), distribution(Dn), collection number (Co.No.) in the environ of Tara-gedam and Amba forests (Continued)

\begin{tabular}{|c|c|c|c|c|c|c|c|c|c|c|c|c|}
\hline \multirow[t]{2}{*}{$\begin{array}{l}\text { Convolvulus } \\
\text { arvensis } \mathrm{L} \text {. }\end{array}$} & \multirow[t]{2}{*}{ Convolvulaceae } & \multirow[t]{2}{*}{ Este filastot } & \multirow[t]{2}{*}{$\mathrm{H}$} & Impotency & $\mathrm{Hu}$ & $\mathrm{R}$ & DF & $\mathrm{O}$ & $\begin{array}{l}\text { Crush and } \\
\text { powder then } \\
\text { drink with } \\
\text { GIN (AREKI) }\end{array}$ & \multirow[t]{2}{*}{ Fwl } & \multirow[t]{2}{*}{$\mathrm{Rr}$} & \multirow[t]{2}{*}{ GC175 } \\
\hline & & & & Anthrax & $\mathrm{Hu}$ & $\mathrm{R}$ & $\mathrm{F}$ & $\mathrm{O}$ & $\begin{array}{l}\text { Peel, chew } \\
\text { then swallow } \\
\text { juice }\end{array}$ & & & \\
\hline $\begin{array}{l}\text { Convolvulus } \\
\text { sagittatus } \\
\text { Thunb. }\end{array}$ & Convolvulaceae & -二-二- & $\mathrm{H}$ & Anthrax & $\mathrm{Hu}$ & $\mathrm{R}$ & $F$ & $\mathrm{O}$ & $\begin{array}{l}\text { Peel, chew } \\
\text { then swallow } \\
\text { juice }\end{array}$ & Ah & $\mathrm{Rr}$ & GC127 \\
\hline \multirow[t]{4}{*}{$\begin{array}{l}\text { Cordia africana } \\
\text { Lam.* }^{*}\end{array}$} & \multirow[t]{4}{*}{ Boraginaceae } & \multirow[t]{4}{*}{ Wanza } & \multirow[t]{4}{*}{$\mathrm{T}$} & Eye problem & $\mathrm{Li}$ & L & DF & Op & $\begin{array}{l}\text { Burn, then } \\
\text { insert ash } \\
\text { with butter }\end{array}$ & \multirow[t]{4}{*}{ Bo } & \multirow[t]{4}{*}{ Wy } & \multirow[t]{4}{*}{ GC133 } \\
\hline & & & & Fire burn & B & L & DF & De & $\begin{array}{l}\text { Burn, then } \\
\text { cream the ash }\end{array}$ & & & \\
\hline & & & & Anthrax & $\mathrm{Li}$ & L & F & $\mathrm{O}$ & $\begin{array}{l}\text { Crush and } \\
\text { give with } \\
\text { water }\end{array}$ & & & \\
\hline & & & & $\begin{array}{l}\text { Expel ear } \\
\text { mites }\end{array}$ & $\mathrm{Hu}$ & $\mathrm{L}$ & $\mathrm{F}$ & Et & $\begin{array}{l}\text { Rub, squeeze, } \\
\text { insert then } \\
\text { cover cotton }\end{array}$ & & & \\
\hline $\begin{array}{l}\text { Crepis rueppellii } \\
\text { Scli-Bip. }\end{array}$ & Asteraceae & -—-—— & $\mathrm{H}$ & Anthrax & $\mathrm{Li}$ & $\mathrm{R}$ & $\mathrm{F}$ & $\mathrm{O}$ & $\begin{array}{l}\text { Crush and } \\
\text { give with } \\
\text { water }\end{array}$ & Fwl & $\mathrm{Rr}$ & GC070 \\
\hline $\begin{array}{l}\text { Crotalaria } \\
\text { karagwensis } \\
\text { Taub. }\end{array}$ & Fabaceae & Yeayt ater & $\mathrm{H}$ & Itchiness & $\mathrm{Hu}$ & $\mathrm{L}$ & $\mathrm{FD}$ & De & $\begin{array}{l}\text { Crush and } \\
\text { powder then } \\
\text { cream with } \\
\text { butter }\end{array}$ & Ah & $\mathrm{Rr}$ & GC051 \\
\hline \multirow[t]{9}{*}{$\begin{array}{l}\text { Croton } \\
\text { macrostachyus } \\
\text { Del. }\end{array}$} & \multirow[t]{9}{*}{ Euphorbiaceae } & \multirow[t]{9}{*}{ Misana } & \multirow[t]{9}{*}{$\mathrm{T}$} & $\begin{array}{l}\text { Intestinal \& } \\
\text { abdominal } \\
\text { problems }\end{array}$ & $\mathrm{Hu}$ & $\mathrm{L}$ & $\mathrm{F}$ & $\mathrm{O}$ & $\begin{array}{l}\text { Boil, grind } \\
\text { then eat with } \\
\text { butter, SHIRRO } \\
\text { or TEFF INJERA }\end{array}$ & \multirow[t]{9}{*}{ Aw } & \multirow[t]{9}{*}{ Wy } & \multirow[t]{9}{*}{ GC130 } \\
\hline & & & & Stomachache & $\mathrm{Hu}$ & Sh & $\mathrm{F}$ & $\mathrm{O}$ & $\begin{array}{l}\text { Drink } \\
\text { concoction }\end{array}$ & & & \\
\hline & & & & Bloating & $\mathrm{Li}$ & Sh & $\mathrm{F}$ & $\mathrm{O}$ & $\begin{array}{l}\text { Crush and } \\
\text { give with } \\
\text { water }\end{array}$ & & & \\
\hline & & & & Ring worm & $\mathrm{Hu}$ & Sp & $F$ & De & $\begin{array}{l}\text { Cream } \\
\text { affected part }\end{array}$ & & & \\
\hline & & & & Evil eye & $\mathrm{Hu}$ & $\mathrm{R}$ & DF & $\begin{array}{l}\text { De } \\
\& O\end{array}$ & $\begin{array}{l}\text { Sniff and } \\
\text { drink the } \\
\text { concoction }\end{array}$ & & & \\
\hline & & & & Snake poison & $\mathrm{Hu}$ & $\mathrm{R}$ & $F$ & $\mathrm{O}$ & $\begin{array}{l}\text { Crush and } \\
\text { drink with } \\
\text { water }\end{array}$ & & & \\
\hline & & & & Tape worm & $\mathrm{Hu}$ & B & $\mathrm{F}$ & $\mathrm{O}$ & $\begin{array}{l}\text { Crush, pound, } \\
\text { then drink } \\
\text { juice }\end{array}$ & & & \\
\hline & & & & Tape worm & $\mathrm{Hu}$ & $\mathrm{L}$ & $\mathrm{F}$ & $\mathrm{O}$ & $\begin{array}{l}\text { Boil, grind, } \\
\text { make it WOTE } \\
\text { (souse) with } \\
\text { butter then } \\
\text { eat with } \\
\text { ENJERA }\end{array}$ & & & \\
\hline & & & & Paralyzed leg & $\mathrm{Hu}$ & $\mathrm{R}$ & DF & De & $\begin{array}{l}\text { Crush with } \\
\text { Carissa }\end{array}$ & & & \\
\hline
\end{tabular}


Table 4 List of plant species used to treat human and livestock ailments: scientific names, family, vernacular name, growth forms (Gf), Ailments treated, Ailment type(At), parts used (Pu), condition of preparation (Cp), route of administration (Ra), method of preparation, habitat (Ha), distribution(Dn), collection number (Co.No.) in the environ of Tara-gedam and Amba forests (Continued)

\begin{tabular}{|c|c|c|c|c|c|c|c|c|c|c|c|c|}
\hline & & & & & & & & & $\begin{array}{l}\text { spinarum root } \\
\text { mix with } \\
\text { water and } \\
\text { immerse } \\
\text { affected part }\end{array}$ & & & \\
\hline \multirow[t]{6}{*}{$\begin{array}{l}\text { Cucumis } \\
\text { ficifolius A. Rich. }\end{array}$} & Cucurbitaceae & Yemidir enbuay & $\mathrm{H}$ & $\begin{array}{l}\text { Bloody } \\
\text { diarrhea }\end{array}$ & B & $\mathrm{R}$ & $\mathrm{F}$ & $\mathrm{O}$ & $\begin{array}{l}\text { Crush and } \\
\text { mix with milk }\end{array}$ & Bo & $\operatorname{Rr}$ & GC139 \\
\hline & & & & Evil eye & $\mathrm{Hu}$ & $\mathrm{R}$ & DF & $\begin{array}{l}\mathrm{Na}, \\
\mathrm{O} \& \\
\mathrm{De}\end{array}$ & $\begin{array}{l}\text { Sniff, drink } \\
\text { and fumigate } \\
\text { concoction }\end{array}$ & & & \\
\hline & & & & $\begin{array}{l}\text { Stomachache } \\
\& \text { Anthrax }\end{array}$ & $\mathrm{Hu}$ & $\mathrm{R}$ & $\mathrm{F}$ & $\mathrm{O}$ & $\begin{array}{l}\text { Peel, chew } \\
\text { then swallow } \\
\text { juice or crush } \\
\text { and drink } \\
\text { with water }\end{array}$ & & & \\
\hline & & & & Evil eye & $\mathrm{Hu}$ & $\begin{array}{l}\mathrm{R} \\
\& \mathrm{Fr}\end{array}$ & FD & $\begin{array}{l}\text { De } \\
\& O\end{array}$ & $\begin{array}{l}\text { Crush and tie } \\
\text { on neck }\end{array}$ & & & \\
\hline & & & & Wound & $\mathrm{Hu}$ & $\mathrm{Fr}$ & $\mathrm{F}$ & De & $\begin{array}{l}\text { Insert the } \\
\text { affect part } \\
\text { into the fruit }\end{array}$ & & & \\
\hline & & & & $\begin{array}{l}\text { Expel ear- } \\
\text { mites }\end{array}$ & $\mathrm{Hu}$ & Sh & $\mathrm{F}$ & Et & $\begin{array}{l}\text { Crush, } \\
\text { squeeze then } \\
\text { insert }\end{array}$ & & & \\
\hline \multirow[t]{3}{*}{$\begin{array}{l}\text { Cucurbita pepo } \\
\text { L. }\end{array}$} & Cucurbitaceae & Duba & $\mathrm{Cl}$ & $\begin{array}{l}\text { Expel } \\
\text { placenta }\end{array}$ & B & $\mathrm{Fr}$ & $\mathrm{F}$ & $\mathrm{O}$ & $\begin{array}{l}\text { Chop then } \\
\text { boil with } \\
\text { water }\end{array}$ & $\mathrm{Hg}$ & Spr & GC166 \\
\hline & & & & $\begin{array}{l}\text { Heart \& } \\
\text { gastritis } \\
\text { problems }\end{array}$ & B & $\mathrm{Fr}$ & $\mathrm{F}$ & $\mathrm{O}$ & $\begin{array}{l}\text { Chop then } \\
\text { boil with } \\
\text { water }\end{array}$ & & & \\
\hline & & & & $\begin{array}{l}\text { Sterile } \\
\text { females }\end{array}$ & $\mathrm{Hu}$ & R & F & $\mathrm{O}$ & $\begin{array}{l}\text { Chew and } \\
\text { swallow juice } \\
\text { to be fertile }\end{array}$ & $\mathrm{F}$ & Wy & GC082 \\
\hline $\begin{array}{l}\text { Cupressus } \\
\text { lusitanica Mill. }\end{array}$ & Cuppressaceae & Yeferenge tid & T & Tooth ach & $\mathrm{Hu}$ & L & $\mathrm{F}$ & $\mathrm{O}$ & $\begin{array}{l}\text { Boil with salt } \\
\text { then take } \\
\text { with teeth }\end{array}$ & & & \\
\hline $\begin{array}{l}\text { Cyathula } \\
\text { prostrata (L.) } \\
\text { Brume }\end{array}$ & Amaranthaceae & Aregist & $\mathrm{H}$ & Anthrax & $\mathrm{Li}$ & L & $\mathrm{F}$ & $\mathrm{O}$ & $\begin{array}{l}\text { Rub, squeeze } \\
\text { then give } \\
\text { with water }\end{array}$ & $\mathrm{Hg}$ & $\mathrm{Pa}$ & GC145 \\
\hline \multirow[t]{2}{*}{$\begin{array}{l}\text { Cynodon } \\
\text { dactylon (L.) } \\
\text { Pers.* }\end{array}$} & Poaceae & Serdo & $\mathrm{H}$ & Snake poison & $\mathrm{Hu}$ & $\mathrm{Ag}$ & $\mathrm{F}$ & $\mathrm{O}$ & $\begin{array}{l}\text { Chew and } \\
\text { absorb the } \\
\text { juice }\end{array}$ & Bo & Wy & GC173 \\
\hline & & & & Tape worm & $\mathrm{Hu}$ & $\begin{array}{l}\text { L\& } \\
\text { St }\end{array}$ & $\mathrm{F}$ & $\mathrm{O}$ & $\begin{array}{l}\text { Drink the } \\
\text { concoction }\end{array}$ & & & \\
\hline \multirow[t]{3}{*}{$\begin{array}{l}\text { Cynoglossum } \\
\text { coeruleum } \\
\text { (Hochst. ex A. } \\
\text { Rich.) DC }\end{array}$} & Boraginaceae & Chegogit & $\mathrm{H}$ & Febrile illness & $\mathrm{Hu}$ & L & $\mathrm{F}$ & $\begin{array}{l}\text { De } \\
\& O\end{array}$ & $\begin{array}{l}\text { Rub, squeeze } \\
\text { then cream } \\
\text { and drink the } \\
\text { juice }\end{array}$ & Bo & Wy & GC114 \\
\hline & & & & $\begin{array}{l}\text { Expel foreign } \\
\text { things from } \\
\text { eye }\end{array}$ & $\mathrm{Hu}$ & L & $\mathrm{F}$ & Op & $\begin{array}{l}\text { Crush } \\
\text { mixture, } \\
\text { squeeze then } \\
\text { insert with } \\
\text { cotton wool }\end{array}$ & & & \\
\hline & & & & Spider poison & $\mathrm{Hu}$ & L & $\mathrm{F}$ & De & $\begin{array}{l}\text { Crush, pound } \\
\text { then cream } \\
\text { with butter }\end{array}$ & & & \\
\hline
\end{tabular}


Table 4 List of plant species used to treat human and livestock ailments: scientific names, family, vernacular name, growth forms (Gf), Ailments treated, Ailment type(At), parts used (Pu), condition of preparation (Cp), route of administration (Ra), method of preparation, habitat (Ha), distribution(Dn), collection number (Co.No.) in the environ of Tara-gedam and Amba forests (Continued)

\begin{tabular}{|c|c|c|c|c|c|c|c|c|c|c|c|c|}
\hline & & & & Wound & $\mathrm{Hu}$ & L & $\mathrm{F}$ & De & $\begin{array}{l}\text { Crush then } \\
\text { cream }\end{array}$ & & & \\
\hline & & & & Eye problem & $\mathrm{Hu}$ & L & $\mathrm{F}$ & Op & $\begin{array}{l}\text { Rub, squeeze } \\
\text { then insert } \\
\text { one-two } \\
\text { droplets }\end{array}$ & & & \\
\hline & & & & $\begin{array}{l}\text { Expel } \\
\text { ear-mites }\end{array}$ & $\mathrm{Hu}$ & L & $\mathrm{F}$ & Et & $\begin{array}{l}\text { Rub, insert } \\
\text { and squeeze }\end{array}$ & & & \\
\hline $\begin{array}{l}\text { Cyperus } \\
\text { dichroostathyus } \\
\text { A.Rich. }\end{array}$ & Cyperaceae & Giramta & $\mathrm{H}$ & Trachoma & $\mathrm{Hu}$ & $\mathrm{Fl}$ & $\mathrm{FD}$ & Op & $\begin{array}{l}\text { Burn and } \\
\text { cream the ash } \\
\text { with butter }\end{array}$ & $\mathrm{F}$ & Wy & GC113 \\
\hline \multirow[t]{2}{*}{$\begin{array}{l}\text { Datura } \\
\text { stramonium L. }\end{array}$} & Solanaceae & Astenagir & $\mathrm{H}$ & $\begin{array}{l}\text { Scabies and } \\
\text { ear wound }\end{array}$ & $\mathrm{Hu}$ & L & $\mathrm{F}$ & De & $\begin{array}{l}\text { Crush then } \\
\text { cream }\end{array}$ & Bo & Wy & GC124 \\
\hline & & & & $\begin{array}{l}\text { Expel foreign } \\
\text { things from } \\
\text { eye }\end{array}$ & $\mathrm{Hu}$ & L & $\mathrm{F}$ & Op & $\begin{array}{l}\text { Crush } \\
\text { mixture, } \\
\text { squeeze then } \\
\text { insert with } \\
\text { cotton wool }\end{array}$ & & & \\
\hline $\begin{array}{l}\text { Dichondra } \\
\text { repens J.R.\&G. } \\
\text { Forst. }\end{array}$ & Convolvulaceae & Afer kocher & $\mathrm{H}$ & Febrile illness & $\mathrm{Hu}$ & L & $\mathrm{F}$ & De & $\begin{array}{l}\text { Rub, squeeze } \\
\text { then cream } \\
\text { except heart }\end{array}$ & Fwl & $\mathrm{Rr}$ & GC180 \\
\hline \multirow[t]{2}{*}{$\begin{array}{l}\text { Diplolophium } \\
\text { africanum } \\
\text { Turcz. }\end{array}$} & Apiaceae & Zegerawta & $\mathrm{H}$ & Headache & $\mathrm{Hu}$ & L & $\mathrm{F}$ & $\mathrm{Na}$ & $\begin{array}{l}\text { Sniff the } \\
\text { unprocessed } \\
\text { leaf }\end{array}$ & $\mathrm{F}$ & $\mathrm{Rr}$ & GC041 \\
\hline & & & & Rabies & $\mathrm{Li}$ & $\mathrm{R}$ & $\mathrm{F}$ & $\mathrm{O}$ & $\begin{array}{l}\text { Pound and } \\
\text { give with } \\
\text { water }\end{array}$ & & & \\
\hline $\begin{array}{l}\text { Dipsacus } \\
\text { pinnatifidus } \\
\text { Steud. ex A. } \\
\text { Rich. }\end{array}$ & Dipsacaceae & Ferezeng/kelem & $\mathrm{H}$ & Rabies & $\mathrm{Hu}$ & L & $\mathrm{F}$ & $\mathrm{Na}$ & $\begin{array}{l}\text { Pound and } \\
\text { give with } \\
\text { water }\end{array}$ & $\mathrm{F}$ & Spr & GC102 \\
\hline $\begin{array}{l}\text { Discopodium } \\
\text { penninervium } \\
\text { Hochst. }\end{array}$ & Solanaceae & Almit & S & $\begin{array}{l}\text { Beating with } \\
\text { stick }\end{array}$ & $\mathrm{Hu}$ & Sh & $\mathrm{F}$ & $\begin{array}{l}\mathrm{Na} \\
\& \mathrm{Et}\end{array}$ & $\begin{array}{l}\text { Crush and } \\
\text { give with } \\
\text { water }\end{array}$ & Fal & $\mathrm{Rr}$ & GC071 \\
\hline \multirow[t]{4}{*}{$\begin{array}{l}\text { Dodonaea } \\
\text { angustifolia L.f. }\end{array}$} & Sapindaceae & Kitkita & S & Scabies & $\mathrm{Hu}$ & L & $\mathrm{F}$ & De & $\begin{array}{l}\text { Crush and } \\
\text { cream with } \\
\text { butter }\end{array}$ & $\mathrm{F}$ & Wy & GC036 \\
\hline & & & & Bone fracture & $\mathrm{Li}$ & $\begin{array}{l}\text { L\& } \\
\text { St }\end{array}$ & $\mathrm{F}$ & De & $\begin{array}{l}\text { Tie twig parts } \\
\text { together }\end{array}$ & & & \\
\hline & & & & Tape worm & $\mathrm{Hu}$ & $\begin{array}{l}R \& \& \\
L\end{array}$ & $\mathrm{~F}$ & $\mathrm{O}$ & $\begin{array}{l}\text { Pound, } \\
\text { immerse in } \\
\text { water and } \\
\text { drink the } \\
\text { diluted } \\
\text { mixture }\end{array}$ & & & \\
\hline & & & & Tape worm & $\mathrm{Hu}$ & $\begin{array}{l}\text { L\& } \\
\text { St }\end{array}$ & $\mathrm{F}$ & $\mathrm{O}$ & $\begin{array}{l}\text { Drink the } \\
\text { concoction }\end{array}$ & & & \\
\hline $\begin{array}{l}\text { Dovyalis } \\
\text { abyssinica (A. } \\
\text { Rich.) Warb.* }\end{array}$ & Flacourtiaceae & Koshim & S & Hemorrhoid & $\mathrm{Hu}$ & $\mathrm{Fr}$ & $\mathrm{F}$ & De & $\begin{array}{l}\text { Immerse in } \\
\text { water in flat } \\
\text { material and } \\
\text { sit on }\end{array}$ & Bo & $\mathrm{Rr}$ & GC042 \\
\hline $\begin{array}{l}\text { Dregea } \\
\text { rubicunda } \\
\text { Schum. }\end{array}$ & Asclepiadaceae & Kuandira & $\mathrm{Cl}$ & Rabies & $\mathrm{Hu}$ & L & $\mathrm{F}$ & O & $\begin{array}{l}\text { Crush and } \\
\text { drink with } \\
\text { milk }\end{array}$ & $\mathrm{F}$ & $\mathrm{Rr}$ & GC044 \\
\hline
\end{tabular}


Table 4 List of plant species used to treat human and livestock ailments: scientific names, family, vernacular name, growth forms (Gf), Ailments treated, Ailment type(At), parts used (Pu), condition of preparation (Cp), route of administration (Ra), method of preparation, habitat (Ha), distribution(Dn), collection number (Co.No.) in the environ of Tara-gedam and Amba forests (Continued)

\begin{tabular}{|c|c|c|c|c|c|c|c|c|c|c|c|c|}
\hline & & & & Wound & $\mathrm{Hu}$ & $\begin{array}{l}\text { L\& } \\
B\end{array}$ & D & De & $\begin{array}{l}\text { Crush, } \\
\text { powder then } \\
\text { tie }\end{array}$ & & & \\
\hline $\begin{array}{l}\text { Dyschoriste } \\
\text { radicans Nees }\end{array}$ & Acanthaceae & -—-二- & $\mathrm{H}$ & Stomachache & $\mathrm{Hu}$ & Ap & $\mathrm{F}$ & $\mathrm{O}$ & $\begin{array}{l}\text { Chew and } \\
\text { swallow the } \\
\text { juice }\end{array}$ & Fwl & $\mathrm{Rr}$ & GC093 \\
\hline $\begin{array}{l}\text { Embelia } \\
\text { schimperi Vatke* }\end{array}$ & Myrsinaceae & Enkoko & S & Tape worm & $\mathrm{Hu}$ & $\mathrm{Fr}$ & FD & $\mathrm{O}$ & $\begin{array}{l}\text { Eat fresh or } \\
\text { crush and } \\
\text { drink with } \\
\text { 'TELA DIFDIF' }\end{array}$ & Ris & $\mathrm{Rr}$ & GC119 \\
\hline \multirow[t]{2}{*}{$\begin{array}{l}\text { Eragrostis tef } \\
\text { (Zucc.) Trotter }\end{array}$} & Poaceae & Tef & $\mathrm{H}$ & Dandruff & $\mathrm{Hu}$ & Sd & D & $\mathrm{De}$ & $\begin{array}{l}\text { Grind, prepare } \\
\text { dough then } \\
\text { cream on } \\
\text { bare head }\end{array}$ & $\mathrm{Hg}$ & Wy & GC040 \\
\hline & & & & Bloating & $\mathrm{Li}$ & Sw & D & $\mathrm{O}$ & $\begin{array}{l}\text { Give the } \\
\text { straw }\end{array}$ & & & \\
\hline $\begin{array}{l}\text { Erythrina } \\
\text { abyssinica Lam. } \\
\text { ex DC. }\end{array}$ & Fabaceae & Kuara & T & Febrile illness & $\mathrm{Li}$ & B & D & $\begin{array}{l}\text { De } \\
\& O\end{array}$ & $\begin{array}{l}\text { Crush then } \\
\text { fumigate or } \\
\text { drink the } \\
\text { concoction }\end{array}$ & Ah & $\mathrm{Rr}$ & GC159 \\
\hline $\begin{array}{l}\text { Eucalyptus } \\
\text { globulus Labill. }\end{array}$ & Myrtaceae & Nech bahirzaf & T & $\begin{array}{l}\text { Febrile illness } \\
\text { \& Common } \\
\text { cold }\end{array}$ & $\mathrm{Hu}$ & L & $\mathrm{F}$ & $\begin{array}{l}\mathrm{Na} \\
\mathrm{O} \& \\
\mathrm{De}\end{array}$ & $\begin{array}{l}\text { Boil and } \\
\text { fumigate with } \\
\text { the fume }\end{array}$ & Fal & $\mathrm{Rr}$ & GC167 \\
\hline \multirow[t]{5}{*}{$\begin{array}{l}\text { Euclea } \\
\text { racemosa Hiern }\end{array}$} & Ebenaceae & Dedeho & S & $\begin{array}{l}\text { Scorpion } \\
\text { poison }\end{array}$ & $\mathrm{Hu}$ & R & $\mathrm{F}$ & $\mathrm{De}$ & Crush and tie & $\mathrm{F}$ & Spr & GC018 \\
\hline & & & & Gonorrhea & $\mathrm{Hu}$ & R & FD & $\mathrm{O}$ & $\begin{array}{l}\text { Boil, crush } \\
\text { then eat with } \\
\text { honey or } \\
\text { butter }\end{array}$ & & & \\
\hline & & & & Eye problem & $\mathrm{Li}$ & $\mathrm{R}$ & $\mathrm{F}$ & Op & $\begin{array}{l}\text { Peel and } \\
\text { cream with } \\
\text { butter for one } \\
\text { night then } \\
\text { use butter for } \\
\text { paint }\end{array}$ & & & \\
\hline & & & & Toothache & $\mathrm{Hu}$ & $\mathrm{Rb}$ & $\mathrm{F}$ & $\mathrm{O}$ & $\begin{array}{l}\text { Take with } \\
\text { teeth }\end{array}$ & & & \\
\hline & & & & $\begin{array}{l}\text { Prolonged } \\
\text { embryo }\end{array}$ & $\mathrm{Hu}$ & $\mathrm{R}$ & DF & De & $\begin{array}{l}\text { Tie the } \\
\text { concoction } \\
\text { on spinal } \\
\text { column }\end{array}$ & & & \\
\hline \multirow[t]{4}{*}{$\begin{array}{l}\text { Euphorbia } \\
\text { abyssinica } \\
\text { Gmel. }\end{array}$} & Euphorbiaceae & Kulkual & T & Jaundice & $\mathrm{Hu}$ & $\mathrm{R}$ & $\mathrm{F}$ & $\mathrm{O}$ & $\begin{array}{l}\text { Crush, } \\
\text { immerse in } \\
\text { water then } \\
\text { drink or bake } \\
\text { with bread } \\
\text { then eat }\end{array}$ & Bo & Wy & GC164 \\
\hline & & & & $\begin{array}{l}\text { Stomach and } \\
\text { intestinal } \\
\text { problems }\end{array}$ & $\mathrm{Hu}$ & $\mathrm{R}$ & $\mathrm{F}$ & $\mathrm{O}$ & $\begin{array}{l}\text { Crush, mix } \\
\text { with DORO } \\
\text { WOTTE then } \\
\text { eat with } \\
\text { ENJERA }\end{array}$ & & & \\
\hline & & & & Rabies & $\mathrm{Li}$ & Lx & F & $\mathrm{O}$ & Mix with milk & & & \\
\hline & & & & Malaria & $\mathrm{Hu}$ & Lx & $\mathrm{F}$ & $\mathrm{O}$ & $\begin{array}{l}\text { Eat bake with } \\
\text { Eragrostis tef } \\
\text { dough }\end{array}$ & & & \\
\hline
\end{tabular}


Table 4 List of plant species used to treat human and livestock ailments: scientific names, family, vernacular name, growth forms (Gf), Ailments treated, Ailment type(At), parts used (Pu), condition of preparation (Cp), route of administration (Ra), method of preparation, habitat (Ha), distribution(Dn), collection number (Co.No.) in the environ of Tara-gedam and Amba forests (Continued)

\begin{tabular}{|c|c|c|c|c|c|c|c|c|c|c|c|c|}
\hline & & & & Hemorrhoid & $\mathrm{Hu}$ & Lx & $\mathrm{F}$ & De & $\begin{array}{l}\text { Cream the } \\
\text { concoction }\end{array}$ & & & \\
\hline & & & & Skin diseases & $\mathrm{Hu}$ & $\mathrm{Fl}$ & $D$ & De & $\begin{array}{l}\text { Crush, } \\
\text { powder, then } \\
\text { cream with } \\
\text { honey }\end{array}$ & & & \\
\hline \multirow[t]{3}{*}{$\begin{array}{l}\text { Euphorbia } \\
\text { tirucalli L. }\end{array}$} & Euphorbiaceae & Kinchib & S & Wound & B & Lx & $\mathrm{F}$ & De & $\begin{array}{l}\text { Paint the } \\
\text { affected part }\end{array}$ & $A h$ & Wy & GC131 \\
\hline & & & & Hemorrhoid & $\mathrm{Hu}$ & Lx & $\mathrm{F}$ & De & $\begin{array}{l}\text { Cream the } \\
\text { concoction }\end{array}$ & & & \\
\hline & & & & Wound & $\mathrm{Hu}$ & Lx & $\mathrm{F}$ & De & $\begin{array}{l}\text { Cream the } \\
\text { concoction }\end{array}$ & & & \\
\hline \multirow[t]{6}{*}{$\begin{array}{l}\text { Ferula } \\
\text { communis L. * }\end{array}$} & Apiaceae & Dog & $\mathrm{H}$ & $\begin{array}{l}\text { Increase } \\
\text { sexual needs }\end{array}$ & $\mathrm{Li}$ & $\mathrm{R}$ & $\mathrm{F}$ & $\mathrm{O}$ & $\begin{array}{l}\text { Pound, then } \\
\text { give with } \\
\text { INGERA and } \\
\text { butter }\end{array}$ & $\mathrm{F}$ & Wy & GC072 \\
\hline & & & & Evil sprit & $\mathrm{Hu}$ & $\mathrm{R}$ & DF & De & $\begin{array}{l}\text { Crush and } \\
\text { fumigate }\end{array}$ & & & \\
\hline & & & & Blood flow & $\mathrm{Hu}$ & $\mathrm{R}$ & $\mathrm{F}$ & $\begin{array}{l}\text { De } \\
\& O\end{array}$ & $\begin{array}{l}\text { Crush, } \\
\text { immerse in } \\
\text { water then } \\
\text { give for } \\
\text { newly } \\
\text { delivered } \\
\text { mother }\end{array}$ & & & \\
\hline & & & & $\begin{array}{l}\text { Lung cancer } \\
\text { (TV) }\end{array}$ & $\mathrm{Hu}$ & $\mathrm{R}$ & $\mathrm{F}$ & $\mathrm{O}$ & $\begin{array}{l}\text { Crush and } \\
\text { drink with } \\
\text { water }\end{array}$ & & & \\
\hline & & & & Erthroblastosis & $\mathrm{Hu}$ & $\mathrm{R}$ & DF & De & $\begin{array}{l}\text { Grind and } \\
\text { drink with } \\
\text { honey or tie } \\
\text { powder } \\
\text { (concoction) } \\
\text { on neck }\end{array}$ & & & \\
\hline & & & & Impotency & $\mathrm{Hu}$ & $\mathrm{R}$ & $\mathrm{F}$ & $\mathrm{O}$ & $\begin{array}{l}\text { Drink } \\
\text { concoction } \\
\text { with honey }\end{array}$ & & & \\
\hline Ficus carica L. & Moraceae & Beles & S & Wound & $\mathrm{Hu}$ & Lx & $\mathrm{F}$ & De & $\begin{array}{l}\text { Cream the } \\
\text { affected part }\end{array}$ & Fwl & $\mathrm{Rr}$ & GC104 \\
\hline Ficus sur Forssk. & Moraceae & Sholla & T & Toothache & $\mathrm{Hu}$ & B & FD & O & Take by teeth & Ris & Spr & GC090 \\
\hline $\begin{array}{l}\text { Ficus vasta } \\
\text { Forssk.* }\end{array}$ & Moraceae & Warka & T & Wound & $\mathrm{Hu}$ & Lx & $\mathrm{F}$ & De & $\begin{array}{l}\text { Cream the } \\
\text { concoction }\end{array}$ & Fal & $\mathrm{Rr}$ & GC162 \\
\hline \multirow[t]{3}{*}{$\begin{array}{l}\text { Foeniculum } \\
\text { vulgare Miller }\end{array}$} & Apiaceae & Ensilal & $\mathrm{H}$ & Cough & $\mathrm{Hu}$ & $\mathrm{Ag}$ & $\mathrm{F}$ & O & $\begin{array}{l}\text { Boil with tea } \\
\text { then drink }\end{array}$ & Bo & $\mathrm{Rr}$ & GC137 \\
\hline & & & & Asma & & $\begin{array}{l}\text { L\& } \\
\text { St }\end{array}$ & $\mathrm{F}$ & $\mathrm{O}$ & $\begin{array}{l}\text { Crush, } \\
\text { immerse with } \\
\text { milk then } \\
\text { drink }\end{array}$ & & & \\
\hline & & & & $\begin{array}{l}\text { Urinary } \\
\text { retention }\end{array}$ & $\mathrm{Hu}$ & $\begin{array}{l}\text { L\& } \\
\text { St }\end{array}$ & $\mathrm{F}$ & $\mathrm{O}$ & $\begin{array}{l}\text { Cook in water } \\
\text { then drink } \\
\text { the juice }\end{array}$ & & & \\
\hline $\begin{array}{l}\text { Gardenia } \\
\text { ternifolia } \\
\text { Schumach. \& } \\
\text { Thonn.* }\end{array}$ & Rubiaceae & Gambillo & T & Erthroblastosis & $\mathrm{Hu}$ & $\mathrm{R}$ & DF & De & $\begin{array}{l}\text { Grind and } \\
\text { drink with } \\
\text { honey or tie } \\
\text { powder/ }\end{array}$ & Bo & $\mathrm{Rr}$ & GC087 \\
\hline
\end{tabular}


Table 4 List of plant species used to treat human and livestock ailments: scientific names, family, vernacular name, growth forms (Gf), Ailments treated, Ailment type(At), parts used (Pu), condition of preparation (Cp), route of administration (Ra), method of preparation, habitat (Ha), distribution(Dn), collection number (Co.No.) in the environ of Tara-gedam and Amba forests (Continued)

\begin{tabular}{|c|c|c|c|c|c|c|c|c|c|c|c|c|}
\hline & & & & & & & & & $\begin{array}{l}\text { concoction } \\
\text { on neck }\end{array}$ & & & \\
\hline \multirow[t]{2}{*}{$\begin{array}{l}\text { Gossypium } \\
\text { barbadense L. }\end{array}$} & Malvaceae & Tit & $S$ & Snake bite & $\mathrm{Hu}$ & $\mathrm{R}$ & DF & $\begin{array}{l}\mathrm{De} \\
\& \mathrm{O}\end{array}$ & $\begin{array}{l}\text { Tie on neck } \\
\text { or chew, } \\
\text { absorb the } \\
\text { juice }\end{array}$ & $\mathrm{Hg}$ & $\operatorname{Rr}$ & GC096 \\
\hline & & & & Tonsillitis & $\mathrm{Hu}$ & $\mathrm{Fr}$ & D & $\mathrm{O}$ & $\begin{array}{l}\text { Grind then } \\
\text { drink the } \\
\text { liquid }\end{array}$ & & & \\
\hline \multirow[t]{2}{*}{$\begin{array}{l}\text { Grewia } \\
\text { ferruginea } \\
\text { Hochst. ex A. } \\
\text { Rich.* }\end{array}$} & Tiliaceae & Lenquata & $S$ & $\begin{array}{l}\text { Expel } \\
\text { placenta }\end{array}$ & $\mathrm{Li}$ & B & $F$ & $\mathrm{O}$ & $\begin{array}{l}\text { Peel the } \\
\text { inside part, } \\
\text { chop, } \\
\text { emulsify with } \\
\text { water then } \\
\text { give }\end{array}$ & $F$ & Wy & GC123 \\
\hline & & & & Dandruff & $\mathrm{Hu}$ & B & $\mathrm{F}$ & $\mathrm{De}$ & $\begin{array}{l}\text { Wash with } \\
\text { inside part }\end{array}$ & & & \\
\hline \multirow[t]{2}{*}{$\begin{array}{l}\text { Guizotia } \\
\text { schimperi Sch. } \\
\text { Bip.ex Walp. }\end{array}$} & Asteraceae & Mech & $\mathrm{H}$ & Stomachache & $\mathrm{Hu}$ & $\mathrm{R}$ & $F$ & $\mathrm{O}$ & $\begin{array}{l}\text { Chew and } \\
\text { swallow the } \\
\text { juice }\end{array}$ & Fwl & Wy & GC073 \\
\hline & & & & Wound & $\mathrm{Li}$ & $\mathrm{Ag}$ & $\mathrm{F}$ & $\mathrm{De}$ & $\begin{array}{l}\text { Rub the part } \\
\text { affected by } \\
\text { ticks }\end{array}$ & & & \\
\hline $\begin{array}{l}\text { Helinus } \\
\text { mystacinus (Ait.) } \\
\text { E. Mey. ex } \\
\text { Steud. }\end{array}$ & Rhamnaceae & Esat abrid & $\mathrm{Cl}$ & Fire burn & $\mathrm{Hu}$ & $L$ & $F$ & De & Crush and tie & $F$ & Spr & GC039 \\
\hline $\begin{array}{l}\text { Heteromorpha } \\
\text { arborescens } \\
\text { (Spreng.) Cham. } \\
\text { \&Schldl. }\end{array}$ & Apiaceae & Yegib mirkuz & $S$ & Snake bite & $\mathrm{Hu}$ & $R$ & $\mathrm{~F}$ & $\begin{array}{l}\text { De } \\
\& O\end{array}$ & $\begin{array}{l}\text { Chew, absorb } \\
\text { and swallow } \\
\text { or tie fresh on } \\
\text { neck }\end{array}$ & Fal & $\operatorname{Rr}$ & GC015 \\
\hline $\begin{array}{l}\text { Hibiscus } \\
\text { macranthus } \\
\text { Hochst. ex A. } \\
\text { Rich. }\end{array}$ & Malvaceae & Nacha & $S$ & Wound & $\mathrm{Hu}$ & $L$ & $F$ & De & $\begin{array}{l}\text { Chew and } \\
\text { cream with } \\
\text { cotton }\end{array}$ & $\mathrm{F}$ & Spr & GC064 \\
\hline $\begin{array}{l}\text { Huernia } \\
\text { macrocarpa (A. } \\
\text { Rich) Sprenger }\end{array}$ & Asclepiadaceae & Yemidir kulkual & $\mathrm{H}$ & $\begin{array}{l}\text { General } \\
\text { medicine }\end{array}$ & $\mathrm{Li}$ & $\mathrm{Ag}$ & $F$ & $\mathrm{O}$ & $\begin{array}{l}\text { Chop and } \\
\text { give or chop } \\
\text { and give after } \\
\text { baking with } \\
\text { black barley }\end{array}$ & Fwl & $\operatorname{Rr}$ & GC100 \\
\hline \multirow[t]{2}{*}{$\begin{array}{l}\text { Hypericum } \\
\text { quartinianum A. } \\
\text { Rich }\end{array}$} & Hypericaceae & Amujia & $S$ & $\begin{array}{l}\text { Urinary } \\
\text { problem }\end{array}$ & $\mathrm{Hu}$ & $\mathrm{R}$ & D & $\mathrm{O}$ & $\begin{array}{l}\text { Crush, } \\
\text { powder then } \\
\text { eat with } \\
\text { honey }\end{array}$ & $\mathrm{F}$ & Spr & GC046 \\
\hline & & & & Stomachache & $\mathrm{Hu}$ & $\mathrm{L}$ & $\mathrm{F}$ & $\mathrm{O}$ & $\begin{array}{l}\text { Chew and } \\
\text { absorb the } \\
\text { liquid }\end{array}$ & & & \\
\hline $\begin{array}{l}\text { Indigofera } \\
\text { arrecta Hochst. } \\
\text { Ex A. Rich. }\end{array}$ & Fabaceae & $-\ldots-1-$ & $\mathrm{H}$ & Snake bite & $\mathrm{Hu}$ & $\mathrm{R}$ & $\mathrm{F}$ & $\mathrm{O}$ & $\begin{array}{l}\text { Chew and } \\
\text { absorb the } \\
\text { juice }\end{array}$ & Fal & $\mathrm{Rr}$ & GC033 \\
\hline \multirow[t]{2}{*}{$\begin{array}{l}\text { Indigofera } \\
\text { prieureana Guill } \\
\text { \&Perr. }\end{array}$} & Fabaceae & - - - - - & $\mathrm{H}$ & $\begin{array}{l}\text { Anthrax \& } \\
\text { Stomach ach }\end{array}$ & $\mathrm{Hu}$ & $\mathrm{R}$ & $\mathrm{F}$ & $\mathrm{O}$ & $\begin{array}{l}\text { Chew and } \\
\text { swallow juice } \\
\text { or crush and } \\
\text { give with } \\
\text { water }\end{array}$ & Fal & Spr & GC125 \\
\hline & Oleaceae & Tenbelel & $S$ & Toothache & $\mathrm{Hu}$ & $\mathrm{R}$ & $\mathrm{F}$ & $\mathrm{O}$ & $\begin{array}{l}\text { Take with } \\
\text { teeth }\end{array}$ & $\mathrm{F}$ & Wy & GC012 \\
\hline
\end{tabular}


Table 4 List of plant species used to treat human and livestock ailments: scientific names, family, vernacular name, growth forms (Gf), Ailments treated, Ailment type(At), parts used (Pu), condition of preparation (Cp), route of administration ( $\mathrm{Ra}$ ), method of preparation, habitat (Ha), distribution(Dn), collection number (Co.No.) in the environ of Tara-gedam and Amba forests (Continued)

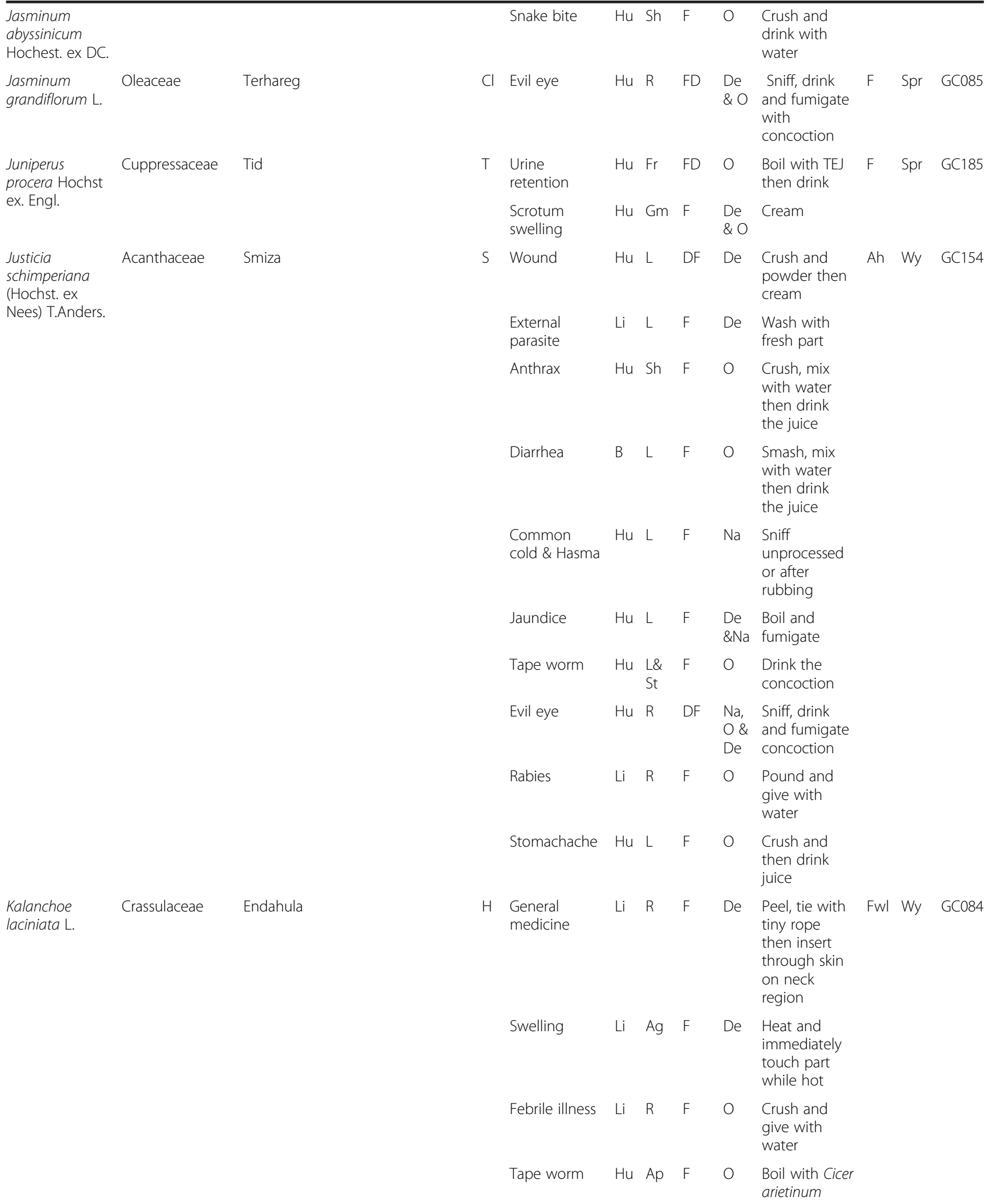


Table 4 List of plant species used to treat human and livestock ailments: scientific names, family, vernacular name, growth forms (Gf), Ailments treated, Ailment type(At), parts used (Pu), condition of preparation (Cp), route of administration (Ra), method of preparation, habitat (Ha), distribution(Dn), collection number (Co.No.) in the environ of Tara-gedam and Amba forests (Continued)

\begin{tabular}{|c|c|c|c|c|c|c|c|c|c|c|c|c|}
\hline & & & & & & & & & $\begin{array}{l}\text { cotyledons } \\
\text { and eat } \\
\text { cotyledons or } \\
\text { crush and mix } \\
\text { with butter } \\
\text { and drink }\end{array}$ & & & \\
\hline \multirow[t]{3}{*}{$\begin{array}{l}\text { Lactuca intermis } \\
\text { Forssk. }\end{array}$} & Asteraceae & Dememerarit & $\mathrm{H}$ & Broken bone & B & $R$ & DF & De & $\begin{array}{l}\text { Tie on the } \\
\text { problematic } \\
\text { part }\end{array}$ & Fal & Wy & GC118 \\
\hline & & & & Amoeba & $\mathrm{Hu}$ & $\mathrm{R}$ & $\mathrm{F}$ & $\mathrm{O}$ & $\begin{array}{l}\text { Chew and } \\
\text { swallow the } \\
\text { juice }\end{array}$ & & & \\
\hline & & & & Wound & B & $L x$ & $\mathrm{~F}$ & De & $\begin{array}{l}\text { Cream after } \\
\text { removing the } \\
\text { ticks }\end{array}$ & & & \\
\hline $\begin{array}{l}\text { Laggera } \\
\text { tomentosa (Sch. } \\
\text { Bip. ex A. Rich.) } \\
\text { Oliv. \& Hiern }\end{array}$ & Asteraceae & Keskeso/Shetie & $\mathrm{H}$ & Swelling & $\mathrm{Hu}$ & L & DF & De & $\begin{array}{l}\text { Rub and tie } \\
\text { or dry, crush, } \\
\text { mix with } \\
\text { honey and } \\
\text { lemon juice } \\
\text { then tie }\end{array}$ & FWl & Wy & GC038 \\
\hline \multirow[t]{4}{*}{$\begin{array}{l}\text { Laggera } \\
\text { crispata (Vahl) } \\
\text { Hepper \& } \\
\text { Wood }\end{array}$} & \multirow[t]{4}{*}{ Asteraceae } & \multirow[t]{4}{*}{ Keskesso/ alshasume } & \multirow[t]{4}{*}{$\mathrm{H}$} & $\begin{array}{l}\text { Gastric \& } \\
\text { Stomachache }\end{array}$ & $\mathrm{Hu}$ & L & $\mathrm{F}$ & $\mathrm{O}$ & $\begin{array}{l}\text { Chew and } \\
\text { swallow the } \\
\text { juice }\end{array}$ & \multirow[t]{4}{*}{ Fal } & \multirow[t]{4}{*}{ Wy } & \multirow[t]{4}{*}{ GC075 } \\
\hline & & & & Tape worm & $\mathrm{Hu}$ & L & $\mathrm{F}$ & $\mathrm{O}$ & $\begin{array}{l}\text { Crush and } \\
\text { drink with } \\
\text { water }\end{array}$ & & & \\
\hline & & & & $\begin{array}{l}\text { Stop blood } \\
\text { flow after } \\
\text { birth }\end{array}$ & $\mathrm{Hu}$ & $\mathrm{R}$ & $\mathrm{F}$ & De & $\begin{array}{l}\text { Crush, } \\
\text { immerse in } \\
\text { water then } \\
\text { spray on } \\
\text { newly } \\
\text { delivered } \\
\text { mother }\end{array}$ & & & \\
\hline & & & & Fire burn & $\mathrm{Hu}$ & L & $\mathrm{F}$ & De & $\begin{array}{l}\text { Rub, squeeze } \\
\text { then cream } \\
\text { with cotton }\end{array}$ & & & \\
\hline $\begin{array}{l}\text { Leonotis } \\
\text { ocymifolia } \\
\text { (Burm.f.) } \\
\text { Iwarsson }\end{array}$ & Lamiaceae & Ferezeng & S & Snake bite & $\mathrm{Hu}$ & $R$ & $\mathrm{~F}$ & De & Crush and tie & $\mathrm{F}$ & $\mathrm{Rr}$ & GC105 \\
\hline $\begin{array}{l}\text { Leucas } \\
\text { martinicensis } \\
\text { (Jaq) R.Br. }\end{array}$ & Lamiaceae & -二-二-二-二 & $\mathrm{H}$ & $\begin{array}{l}\text { Prevent } \\
\text { diseases } \\
\text { relapse }\end{array}$ & $\mathrm{Hu}$ & $\mathrm{Ag}$ & DF & De & $\begin{array}{l}\text { Fumigate the } \\
\text { fume }\end{array}$ & $\mathrm{F}$ & $\mathrm{Rr}$ & GC053 \\
\hline $\begin{array}{l}\text { Linum } \\
\text { usitatissimum L. }\end{array}$ & Linaceae & Telba & $\mathrm{H}$ & Wound & $\mathrm{Hu}$ & $\mathrm{R}$ & $\mathrm{D}$ & De & $\begin{array}{l}\text { Crush, mix } \\
\text { with honey } \\
\text { then cream }\end{array}$ & Fal & Spr & GC184 \\
\hline $\begin{array}{l}\text { Maesa laceolata } \\
\text { Forssk. }\end{array}$ & Myrsinaceae & Kilabo & S & Womb & $\mathrm{Hu}$ & $\mathrm{Fr}$ & $D$ & Va & $\begin{array}{l}\text { Roast, grind, } \\
\text { mix with } \\
\text { butter then } \\
\text { cream }\end{array}$ & $\mathrm{F}$ & Spr & GC068 \\
\hline $\begin{array}{l}\text { Malva } \\
\text { verticillata } \mathrm{L} .\end{array}$ & Malvaceae & Elit & $\mathrm{H}$ & Scabies & $\mathrm{Hu}$ & $\mathrm{Ag}$ & DF & De & $\begin{array}{l}\text { Crush, } \\
\text { powder and } \\
\text { tie }\end{array}$ & $A h$ & $\mathrm{Rr}$ & GC103 \\
\hline \multirow[t]{2}{*}{$\begin{array}{l}\text { Melia azedarach } \\
\text { L. }\end{array}$} & \multirow[t]{2}{*}{ Meliaceae } & \multirow[t]{2}{*}{$\mathrm{Nim}$} & \multirow[t]{2}{*}{$\mathrm{T}$} & \multirow[t]{2}{*}{ Dandruff } & $\mathrm{Hu}$ & $L$ & $\mathrm{~F}$ & De & $\begin{array}{l}\text { Crush and } \\
\text { cream }\end{array}$ & $\mathrm{Hg}$ & Spr & GC160 \\
\hline & & & & & $\mathrm{Hu}$ & L & DF & De & & & & \\
\hline
\end{tabular}


Table 4 List of plant species used to treat human and livestock ailments: scientific names, family, vernacular name, growth forms (Gf), Ailments treated, Ailment type(At), parts used (Pu), condition of preparation (Cp), route of administration (Ra), method of preparation, habitat (Ha), distribution(Dn), collection number (Co.No.) in the environ of Tara-gedam and Amba forests (Continued)

\begin{tabular}{|c|c|c|c|c|c|c|c|c|c|c|c|c|}
\hline & & & & $\begin{array}{l}\text { Anti- } \\
\text { insecticide }\end{array}$ & & & & & $\begin{array}{l}\text { Crush and } \\
\text { powder, then } \\
\text { spray with } \\
\text { water }\end{array}$ & & & \\
\hline \multirow[t]{2}{*}{$\begin{array}{l}\text { Millettia } \\
\text { ferruginea } \\
\text { (Hochst.) Bak. }\end{array}$} & Fabaceae & Birbira & T & Leeches & $\mathrm{Li}$ & L & $\mathrm{F}$ & O & $\begin{array}{l}\text { Crush and } \\
\text { give with } \\
\text { water }\end{array}$ & $\mathrm{F}$ & $\mathrm{Rr}$ & GC067 \\
\hline & & & & Rabies & $\mathrm{Li}$ & St & DF & De & $\begin{array}{l}\text { Heat stick } \\
\text { then touch } \\
\text { their body } \\
\text { with hot part }\end{array}$ & & & \\
\hline $\begin{array}{l}\text { Mimusops } \\
\text { kummel A.DC.* }\end{array}$ & Sapotaceae & Eshe & T & Hasma & $\mathrm{Hu}$ & $\mathrm{Fr}$ & $\mathrm{F}$ & $\mathrm{O}$ & Eat raw fruit & Ris & $\mathrm{Rr}$ & GC101 \\
\hline \multirow[t]{4}{*}{$\begin{array}{l}\text { Momordica } \\
\text { foetida } \\
\text { Schumach. }\end{array}$} & Cucurbitaceae & Yekurahareg/Kuramechat & $\mathrm{H}$ & $\begin{array}{l}\text { Diarrhea \& } \\
\text { gonorrhea }\end{array}$ & $\mathrm{Hu}$ & L & $\mathrm{F}$ & $\mathrm{O}$ & $\begin{array}{l}\text { Pound, } \\
\text { squeeze then } \\
\text { drink }\end{array}$ & $\mathrm{F}$ & Spr & GC165 \\
\hline & & & & Tonsillitis & $\mathrm{Hu}$ & L & $\mathrm{F}$ & O & $\begin{array}{l}\text { Pound, } \\
\text { squeeze then } \\
\text { drink }\end{array}$ & & & \\
\hline & & & & Sun stroke & $\mathrm{Li}$ & L & $\mathrm{F}$ & $\mathrm{O}$ & $\begin{array}{l}\text { Crush and } \\
\text { give with } \\
\text { water }\end{array}$ & & & \\
\hline & & & & Evil sprit & $\mathrm{Hu}$ & $\begin{array}{l}\text { L\& } \\
R\end{array}$ & $\mathrm{~F}$ & De & $\begin{array}{l}\text { Boil and } \\
\text { fumigate }\end{array}$ & & & \\
\hline \multirow[t]{2}{*}{$\begin{array}{l}\text { Myrica salicifolia } \\
\text { Hochst. ex A. } \\
\text { Rich. }\end{array}$} & Myricaceae & Shinet & T & $\begin{array}{l}\text { Common } \\
\text { cold \& } \\
\text { bleeding }\end{array}$ & $\mathrm{Hu}$ & B & FD & $\mathrm{Na}$ & $\begin{array}{l}\text { Crush, } \\
\text { powder then } \\
\text { sniff }\end{array}$ & Ris & $\mathrm{Rr}$ & GC106 \\
\hline & & & & Eye problem & $\mathrm{Li}$ & B & FD & Op & $\begin{array}{l}\text { Crush, } \\
\text { powder then } \\
\text { insert }\end{array}$ & & & \\
\hline $\begin{array}{l}\text { Nicandra } \\
\text { physaloides (L.) } \\
\text { Gaertn. }\end{array}$ & Solanaceae & Kassa & $\mathrm{H}$ & Fire burn & $\mathrm{Hu}$ & $\mathrm{L}$ & $\mathrm{F}$ & De & $\begin{array}{l}\text { Crush, mix } \\
\text { with butter } \\
\text { then cream }\end{array}$ & Fal & Spr & GC065 \\
\hline $\begin{array}{l}\text { Nicotiana } \\
\text { tabacum L. }\end{array}$ & Solanaceae & Tinbaho & S & Wound & $\mathrm{Hu}$ & L & $D$ & De & $\begin{array}{l}\text { Crush and } \\
\text { powder then } \\
\text { cream }\end{array}$ & $\mathrm{Hg}$ & $\mathrm{Rr}$ & GC080 \\
\hline $\begin{array}{l}\text { Nuxia congesta } \\
\text { R.Br. ex Fresen. }\end{array}$ & Loganiaceae & Atquar & S & Tonsillitis & $\mathrm{Hu}$ & Sh & $\mathrm{F}$ & $\begin{array}{l}\text { De } \\
\& O\end{array}$ & $\begin{array}{l}\text { Rub, squeeze } \\
\text { then drink } \\
\text { and put on } \\
\text { head }\end{array}$ & $\mathrm{F}$ & Spr & GC088 \\
\hline \multirow{2}{*}{$\begin{array}{l}\text { Ocimum } \\
\text { urticifolium } \\
\text { Koth }\end{array}$} & Lamiaceae & Dama kesie & S & Febrile illness & $\mathrm{Hu}$ & L & $\mathrm{F}$ & $\mathrm{O}$ & $\begin{array}{l}\text { Boil with tea } \\
\text { and drink }\end{array}$ & $\mathrm{Hg}$ & Spr & GC129 \\
\hline & & & & $\begin{array}{l}\text { Common } \\
\text { cold }\end{array}$ & $\mathrm{Hu}$ & $\mathrm{L}$ & $\mathrm{F}$ & $\mathrm{O}$ & $\begin{array}{l}\text { Boil with tea } \\
\text { and drink }\end{array}$ & & & \\
\hline \multirow{4}{*}{$\begin{array}{l}\text { Olea europaea } \\
\text { L. subsp. } \\
\text { cuspidata (Wall. } \\
\text { ex G. Don) Cif. }\end{array}$} & Oleaceae & Woira & $\mathrm{T}$ & Tonsillitis & $\mathrm{Hu}$ & L & $\mathrm{F}$ & $\mathrm{O}$ & $\begin{array}{l}\text { Chew and } \\
\text { absorb the } \\
\text { juice }\end{array}$ & $\mathrm{Ft}$ & Wy & GC079 \\
\hline & & & & Evil eye & $\mathrm{Hu}$ & St & $\mathrm{F}$ & De & $\begin{array}{l}\text { Beating with } \\
\text { fresh stick }\end{array}$ & & & \\
\hline & & & & Eye diseases & $\mathrm{Hu}$ & L & $\mathrm{F}$ & Op & $\begin{array}{l}\text { Pound, } \\
\text { squeeze then } \\
\text { drop with } \\
\text { cotton }\end{array}$ & & & \\
\hline & & & & Deafness & $\mathrm{Hu}$ & L & $\mathrm{F}$ & Et & $\begin{array}{l}\text { Drop } \\
\text { concoction } \\
\text { with food oil }\end{array}$ & & & \\
\hline
\end{tabular}


Table 4 List of plant species used to treat human and livestock ailments: scientific names, family, vernacular name, growth forms (Gf), Ailments treated, Ailment type(At), parts used (Pu), condition of preparation (Cp), route of administration (Ra), method of preparation, habitat (Ha), distribution(Dn), collection number (Co.No.) in the environ of Tara-gedam and Amba forests (Continued)

\begin{tabular}{|c|c|c|c|c|c|c|c|c|c|c|c|c|}
\hline $\begin{array}{l}\text { Ormocarpum } \\
\text { pubescens } \\
\text { (Hochst.) Cuf.ex. } \\
\text { Gillett }\end{array}$ & Fabaceae & Murna & $S$ & Wound & $\mathrm{Hu}$ & $\mathrm{L}$ & DF & De & $\begin{array}{l}\text { Crush, } \\
\text { powder then } \\
\text { tie }\end{array}$ & $F$ & $\mathrm{Rr}$ & GC014 \\
\hline $\begin{array}{l}\text { Orobanche } \\
\text { ramosa L. }\end{array}$ & Orobanchaceae & -- - & $\mathrm{H}$ & Sunstroke & $\mathrm{Li}$ & Ap & D & De & Fumigate & Fwl & $\mathrm{Rr}$ & GC181 \\
\hline \multirow{3}{*}{$\begin{array}{l}\text { Otostegia } \\
\text { integrifolia } \\
\text { Benth. }\end{array}$} & \multirow[t]{3}{*}{ Lamiaceae } & \multirow[t]{3}{*}{ Tunjut } & \multirow[t]{3}{*}{ S } & $\begin{array}{l}\text { Epidemic \& } \\
\text { common cold }\end{array}$ & $\mathrm{Hu}$ & $\mathrm{Ag}$ & D & De & $\begin{array}{l}\text { Fumigate the } \\
\text { house }\end{array}$ & \multirow[t]{3}{*}{$\mathrm{F}$} & \multirow[t]{3}{*}{ Spr } & \multirow[t]{3}{*}{ GC141 } \\
\hline & & & & Coccolida & $\mathrm{Li}$ & $\mathrm{Ag}$ & $\mathrm{D}$ & De & Fumigate & & & \\
\hline & & & & Stomachache & $\mathrm{Hu}$ & Sh & $\mathrm{F}$ & $\mathrm{O}$ & $\begin{array}{l}\text { Rub, squeeze } \\
\text { then drink } \\
\text { liquid }\end{array}$ & & & \\
\hline $\begin{array}{l}\text { Pentas } \\
\text { lanceolata } \\
\text { (Forssk.) Defl. }\end{array}$ & Rubiaceae & Ras faris & $S$ & Tite problem & $\mathrm{Li}$ & $\mathrm{L}$ & $\mathrm{F}$ & De & $\begin{array}{l}\text { Crush, } \\
\text { powder then } \\
\text { cream }\end{array}$ & $\mathrm{F}$ & $\mathrm{Rr}$ & GC066 \\
\hline \multirow[t]{2}{*}{$\begin{array}{l}\text { Periploca } \\
\text { linearifolia } \\
\text { Quant. Dill. \& } \\
\text { Rich. }\end{array}$} & \multirow[t]{2}{*}{ Asclepiadaceae } & \multirow[t]{2}{*}{ Moider } & \multirow[t]{2}{*}{$\mathrm{Cl}$} & Hemorrhoid & $\mathrm{Hu}$ & St & $\mathrm{F}$ & De & $\begin{array}{l}\text { Heat with fire } \\
\text { then } \\
\text { immediately } \\
\text { apply }\end{array}$ & \multirow[t]{2}{*}{$\mathrm{F}$} & \multirow[t]{2}{*}{ Spr } & \multirow[t]{2}{*}{ GC150 } \\
\hline & & & & Hemorrhoid & $\mathrm{Hu}$ & $\mathrm{R}$ & $\mathrm{F}$ & De & Crush and tie & & & \\
\hline $\begin{array}{l}\text { Persea } \\
\text { americana Mill. }\end{array}$ & Lauraceae & Avocado & S & $\begin{array}{l}\text { Kidney } \\
\text { infection }\end{array}$ & $\mathrm{Hu}$ & $\mathrm{L}$ & $\mathrm{F}$ & $\mathrm{O}$ & $\begin{array}{l}\text { Boil and drink } \\
\text { juice }\end{array}$ & $\mathrm{Hg}$ & $\mathrm{Rr}$ & GC183 \\
\hline $\begin{array}{l}\text { Phyllanthus } \\
\text { rotundifolius } \\
\text { Willd. }\end{array}$ & Euphorbiaceae & -——-——— & $\mathrm{H}$ & Ring worm & $\mathrm{Hu}$ & $L x$ & $\mathrm{~F}$ & De & Cream & Fal & $\mathrm{Rr}$ & GC019 \\
\hline \multirow[t]{8}{*}{$\begin{array}{l}\text { Phytolacca } \\
\text { dodecandra } \\
\text { L'Herit. }\end{array}$} & \multirow[t]{8}{*}{ Phytolaccaceae } & \multirow[t]{8}{*}{ Endod } & \multirow[t]{8}{*}{ S } & Leeches & $\mathrm{Li}$ & $\mathrm{L}$ & $\mathrm{F}$ & $\mathrm{Na}$ & $\begin{array}{l}\text { Crush and } \\
\text { insert with } \\
\text { water }\end{array}$ & \multirow[t]{8}{*}{ Bo } & \multirow[t]{8}{*}{ Spr } & \multirow[t]{8}{*}{ GC024 } \\
\hline & & & & Jaundice & $\mathrm{Hu}$ & $\mathrm{L}$ & $\mathrm{F}$ & $\mathrm{O}$ & $\begin{array}{l}\text { Crush and } \\
\text { drink with } \\
\text { water }\end{array}$ & & & \\
\hline & & & & $\begin{array}{l}\text { External } \\
\text { parasite }\end{array}$ & $\mathrm{Li}$ & $L$ & $\mathrm{~F}$ & De & $\begin{array}{l}\text { Wash with } \\
\text { unprocessed } \\
\text { leaf }\end{array}$ & & & \\
\hline & & & & Rabies & $\mathrm{Li}$ & $\mathrm{R}$ & $\mathrm{F}$ & $\mathrm{O}$ & $\begin{array}{l}\text { Crush and } \\
\text { give with milk }\end{array}$ & & & \\
\hline & & & & Elephantiasis & $\mathrm{Hu}$ & $\mathrm{L}$ & $\mathrm{F}$ & De & $\begin{array}{l}\text { Crush, decant, } \\
\text { and insert } \\
\text { juice }\end{array}$ & & & \\
\hline & & & & Malaria & $\mathrm{Hu}$ & $\mathrm{R}$ & $\mathrm{F}$ & $\mathrm{O}$ & $\begin{array}{l}\text { Crush, } \\
\text { squeeze then } \\
\text { drink }\end{array}$ & & & \\
\hline & & & & Anthrax & $\mathrm{Hu}$ & Sh & $\mathrm{F}$ & $\mathrm{O}$ & $\begin{array}{l}\text { Crush, mix } \\
\text { with water } \\
\text { then drink }\end{array}$ & & & \\
\hline & & & & Coccinia & $\mathrm{Li}$ & $\mathrm{R}$ & $\mathrm{F}$ & $\mathrm{O}$ & $\begin{array}{l}\text { Crush, } \\
\text { immerse in } \\
\text { water then } \\
\text { give }\end{array}$ & & & \\
\hline $\begin{array}{l}\text { Plantago } \\
\text { lanceolata L. }\end{array}$ & Plantaginaceae & Wonberet/ Gorteb & $\mathrm{H}$ & $\begin{array}{l}\text { Wound \& } \\
\text { bleeding }\end{array}$ & $\mathrm{Hu}$ & $\mathrm{L}$ & DF & De & $\begin{array}{l}\text { Crush, } \\
\text { powder then } \\
\text { cream }\end{array}$ & Fal & Wy & GC117 \\
\hline
\end{tabular}


Table 4 List of plant species used to treat human and livestock ailments: scientific names, family, vernacular name, growth forms (Gf), Ailments treated, Ailment type(At), parts used (Pu), condition of preparation (Cp), route of administration (Ra), method of preparation, habitat (Ha), distribution(Dn), collection number (Co.No.) in the environ of Tara-gedam and Amba forests (Continued)

\begin{tabular}{|c|c|c|c|c|c|c|c|c|c|c|c|c|}
\hline $\begin{array}{l}\text { Plectranthus } \\
\text { tenuiflorus } \\
\text { (vatke) Agnew }\end{array}$ & Lamiaceae & Mutansa & $S$ & $\begin{array}{l}\text { Weaken } \\
\text { babies \& evil } \\
\text { sprit }\end{array}$ & $\mathrm{Hu}$ & Ap & DF & $\mathrm{O}$ & $\begin{array}{l}\text { Crush, } \\
\text { powder then } \\
\text { give with } \\
\text { water }\end{array}$ & $\mathrm{Hg}$ & $\mathrm{Rr}$ & GC148 \\
\hline \multirow[t]{2}{*}{$\begin{array}{l}\text { Plumbago } \\
\text { zeylanica L. }\end{array}$} & \multirow[t]{2}{*}{ plumbaginaceae } & \multirow[t]{2}{*}{ Amera } & \multirow[t]{2}{*}{$\mathrm{H}$} & Wound & $\mathrm{Hu}$ & $\mathrm{R}$ & DF & De & $\begin{array}{l}\text { Cream } \\
\text { concoction }\end{array}$ & \multirow[t]{2}{*}{ Fwl } & \multirow[t]{2}{*}{$\mathrm{Rr}$} & \multirow[t]{2}{*}{ GC128 } \\
\hline & & & & $\begin{array}{l}\text { Stomachache } \\
\& \text { Scorpion } \\
\text { poison }\end{array}$ & $\mathrm{Hu}$ & $\begin{array}{l}L \& \\
R\end{array}$ & $\mathrm{~F}$ & $\mathrm{O}$ & $\begin{array}{l}\text { Crush and } \\
\text { drink with } \\
\text { water }\end{array}$ & & & \\
\hline \multirow[t]{3}{*}{$\begin{array}{l}\text { Premna } \\
\text { schimperi Engl. }\end{array}$} & \multirow[t]{3}{*}{ Lamiaceae } & \multirow[t]{3}{*}{ Chocho } & \multirow[t]{3}{*}{$S$} & Eye problem & $\mathrm{Li}$ & $L$ & $\mathrm{~F}$ & Op & $\begin{array}{l}\text { Chew and } \\
\text { spit }\end{array}$ & \multirow[t]{3}{*}{$\mathrm{F}$} & \multirow[t]{3}{*}{ Spr } & \multirow[t]{3}{*}{ GC126 } \\
\hline & & & & Wound & $\mathrm{Hu}$ & $\begin{array}{l}B \& \\
L\end{array}$ & D & De & $\begin{array}{l}\text { Crush, } \\
\text { powder then } \\
\text { cream with } \\
\text { butter or } \\
\text { honey }\end{array}$ & & & \\
\hline & & & & Toothache & $\mathrm{Hu}$ & $\mathrm{R}$ & $\mathrm{F}$ & $\mathrm{O}$ & $\begin{array}{l}\text { Chew and } \\
\text { take with } \\
\text { teeth }\end{array}$ & & & \\
\hline \multirow[t]{2}{*}{$\begin{array}{l}\text { Prunus persica } \\
\text { (L.) Batsch }\end{array}$} & \multirow[t]{2}{*}{ Rosaceae } & \multirow[t]{2}{*}{ Kok } & \multirow[t]{2}{*}{$S$} & Diarrhea & $\mathrm{Li}$ & $L$ & $\mathrm{~F}$ & $\mathrm{O}$ & $\begin{array}{l}\text { Crush, } \\
\text { immerse in } \\
\text { water then } \\
\text { give }\end{array}$ & \multirow[t]{2}{*}{$\mathrm{Hg}$} & \multirow[t]{2}{*}{$\mathrm{Rr}$} & \multirow[t]{2}{*}{ GC049 } \\
\hline & & & & Tape worm & $\mathrm{Hu}$ & $\begin{array}{l}\text { L\& } \\
\text { St }\end{array}$ & $\mathrm{F}$ & $\mathrm{O}$ & $\begin{array}{l}\text { Drink the } \\
\text { concoction }\end{array}$ & & & \\
\hline $\begin{array}{l}\text { Punica } \\
\text { granatum L. }\end{array}$ & Punicaceae & Roman & $S$ & $\begin{array}{l}\text { Cancer \& skin } \\
\text { diseases }\end{array}$ & $\mathrm{Hu}$ & $\mathrm{Fr}$ & $\mathrm{F}$ & $\mathrm{O}$ & Crush and eat & $\mathrm{Hg}$ & $\mathrm{Pa}$ & GC022 \\
\hline \multirow[t]{2}{*}{$\begin{array}{l}\text { Rhamnus } \\
\text { prinoides L'Herit }\end{array}$} & \multirow[t]{2}{*}{ Rhamnaceae } & \multirow[t]{2}{*}{ Gesho } & \multirow[t]{2}{*}{$S$} & Tonsillitis & $\mathrm{Hu}$ & Sh & $\mathrm{F}$ & 0 & $\begin{array}{l}\text { Crush and } \\
\text { drink with } \\
\text { water }\end{array}$ & \multirow[t]{2}{*}{$\mathrm{Hg}$} & \multirow[t]{2}{*}{ Spr } & \multirow[t]{2}{*}{ GC094 } \\
\hline & & & & Herpes & $\mathrm{Hu}$ & $\mathrm{L}$ & $\mathrm{F}$ & De & $\begin{array}{l}\text { Grind and } \\
\text { cream }\end{array}$ & & & \\
\hline $\begin{array}{l}\text { Ricinus } \\
\text { communis L. }\end{array}$ & Euphorbiaceae & Chakima/ Gulo & $S$ & Calf diarrhea & $\mathrm{Li}$ & $\mathrm{Fr}$ & F & $\mathrm{O}$ & $\begin{array}{l}\text { Pound cream } \\
\text { the teat of } \\
\text { cow then } \\
\text { allow to suck }\end{array}$ & $\mathrm{Hg}$ & $\mathrm{Rr}$ & GC170 \\
\hline $\begin{array}{l}\text { Rosa abyssinica } \\
\text { Lindley* }^{*}\end{array}$ & Rosaceae & Kega & $S$ & $\begin{array}{l}\text { Tension/ } \\
\text { dizziness }\end{array}$ & $\mathrm{Hu}$ & $\mathrm{Fr}$ & $F$ & $\mathrm{O}$ & $\begin{array}{l}\text { Eat the raw } \\
\text { fruit }\end{array}$ & $F$ & Spr & GC037 \\
\hline $\begin{array}{l}\text { Rubia cordifolia } \\
\text { L. }\end{array}$ & Rubiaceae & Mencherer & $\mathrm{Cl}$ & Cough & $\mathrm{Hu}$ & $\begin{array}{l}R \& \\
L\end{array}$ & $\mathrm{~F}$ & $\mathrm{O}$ & $\begin{array}{l}\text { Drink the } \\
\text { concoction } \\
\text { with tea or } \\
\text { coffee }\end{array}$ & $\mathrm{F}$ & $\mathrm{Rr}$ & GC110 \\
\hline $\begin{array}{l}\text { Rumex } \\
\text { abyssinicus } \\
\text { Jacq.* }\end{array}$ & Polygonaceae & Mekmoko & $\mathrm{H}$ & Hypertension & $\mathrm{Hu}$ & $\mathrm{R}$ & DF & $\mathrm{O}$ & $\begin{array}{l}\text { Pound, } \\
\text { powder then } \\
\text { drink with } \\
\text { milk }\end{array}$ & Fal & Spr & GC076 \\
\hline \multirow[t]{2}{*}{$\begin{array}{l}\text { Rumex } \\
\text { nepalensis } \\
\text { Spreng. }\end{array}$} & \multirow[t]{2}{*}{ Polygonaceae } & \multirow[t]{2}{*}{ Tult } & \multirow[t]{2}{*}{$\mathrm{H}$} & $\begin{array}{l}\text { Tonsillitis \& } \\
\text { diarrhea }\end{array}$ & $\mathrm{Hu}$ & $\mathrm{R}$ & DF & De & $\begin{array}{l}\text { Crush, mix } \\
\text { with water } \\
\text { then drink } \\
\text { juice or tie on } \\
\text { neck without } \\
\text { processing }\end{array}$ & \multirow[t]{2}{*}{ Fwl } & \multirow[t]{2}{*}{ Spr } & GC029 \\
\hline & & & & Stomachache & $\mathrm{Hu}$ & $\mathrm{R}$ & DF & $\mathrm{O}$ & $\begin{array}{l}\text { Chew and } \\
\text { swallow the } \\
\text { juice }\end{array}$ & & & \\
\hline
\end{tabular}


Table 4 List of plant species used to treat human and livestock ailments: scientific names, family, vernacular name, growth forms (Gf), Ailments treated, Ailment type(At), parts used (Pu), condition of preparation (Cp), route of administration (Ra), method of preparation, habitat (Ha), distribution(Dn), collection number (Co.No.) in the environ of Tara-gedam and Amba forests (Continued)

\begin{tabular}{|c|c|c|c|c|c|c|c|c|c|c|c|c|}
\hline & & & & Anthrax & $\mathrm{Li}$ & $\mathrm{R}$ & $\mathrm{F}$ & $\mathrm{O}$ & $\begin{array}{l}\text { Crush and } \\
\text { give with } \\
\text { water }\end{array}$ & & & \\
\hline \multirow[t]{2}{*}{$\begin{array}{l}\text { Rumex nervosus } \\
\text { Vahl* }\end{array}$} & Polygonaceae & Enbuacho & S & Wart & $\mathrm{Hu}$ & L & $\mathrm{F}$ & De & $\begin{array}{l}\text { Rub, squeeze } \\
\text { then cream }\end{array}$ & Fal & Wy & GC177 \\
\hline & & & & $\begin{array}{l}\text { Bleeding } \\
\text { wound }\end{array}$ & $\mathrm{Hu}$ & L & $\mathrm{F}$ & De & $\begin{array}{l}\text { Pound then } \\
\text { tie }\end{array}$ & & & \\
\hline \multirow[t]{2}{*}{$\begin{array}{l}\text { Ruta } \\
\text { chalepensis L. }\end{array}$} & Rutaceae & Tenadam & $\mathrm{H}$ & Evil eye & $\mathrm{Hu}$ & L & DF & $\begin{array}{l}\text { De } \\
\& O\end{array}$ & $\begin{array}{l}\text { Sniff, drink } \\
\text { and fumigate } \\
\text { with } \\
\text { concoction }\end{array}$ & $\mathrm{Hg}$ & $\mathrm{Rr}$ & GC186 \\
\hline & & & & Febrile illness & $\mathrm{Hu}$ & L & $\mathrm{F}$ & $\mathrm{O}$ & $\begin{array}{l}\text { Crush then } \\
\text { fumigate } \\
\text { whole body } \\
\text { or drink the } \\
\text { concoction }\end{array}$ & & & \\
\hline $\begin{array}{l}\text { Sansevieria } \\
\text { erythraeae } \\
\text { Mattei }\end{array}$ & Dracaenaceae & Chiret & S & Ear wound & $\mathrm{Hu}$ & St & $\mathrm{F}$ & Et & $\begin{array}{l}\text { Heat, pound, } \\
\text { squeeze then } \\
\text { insert while } \\
\text { cool }\end{array}$ & $\mathrm{Hg}$ & $\mathrm{Rr}$ & GC111 \\
\hline $\begin{array}{l}\text { Schefflera } \\
\text { abyssinica } \\
\text { (Hochst. ex A. } \\
\text { Rich.) Harms. }\end{array}$ & Araliaceae & Getem & $\mathrm{T}$ & Snake poison & $\mathrm{Hu}$ & B & $F$ & $\mathrm{O}$ & $\begin{array}{l}\text { Crush and } \\
\text { drink the } \\
\text { infusion }\end{array}$ & $\mathrm{F}$ & $\mathrm{Rr}$ & GC171 \\
\hline \multirow[t]{2}{*}{ Schinus molle L. } & Anacardiaceae & Kundoberbere & $\mathrm{T}$ & Cough & $\mathrm{Hu}$ & $\mathrm{Fr}$ & DF & $\mathrm{O}$ & $\begin{array}{l}\text { Pound, cook } \\
\text { in DORRO } \\
\text { WOTE then } \\
\text { eat with TEF } \\
\text { ENGERA }\end{array}$ & $\mathrm{Hg}$ & Spr & GC155 \\
\hline & & & & Wound & $\mathrm{Hu}$ & L & $F$ & $\mathrm{De}$ & $\begin{array}{l}\text { Pound and } \\
\text { tie }\end{array}$ & & & \\
\hline $\begin{array}{l}\text { Senna } \\
\text { didymobotrya } \\
\text { (Fresen.) Irwin } \\
\text { \&Bameby }\end{array}$ & Fabaceae & Serka Abeba & S & Bloating & $\mathrm{Li}$ & L & $\mathrm{F}$ & $\mathrm{O}$ & $\begin{array}{l}\text { Crush and } \\
\text { give with } \\
\text { water }\end{array}$ & $\mathrm{Fwl}$ & Wy & GC122 \\
\hline $\begin{array}{l}\text { Sida ovata } \\
\text { Forssk. }\end{array}$ & Malvaceae & Yahya-nacha & $\mathrm{H}$ & Fire burn & $\mathrm{Hu}$ & $\mathrm{R}$ & $\mathrm{F}$ & De & $\begin{array}{l}\text { Pound and } \\
\text { cream the } \\
\text { liquid with } \\
\text { cotton }\end{array}$ & Fal & Spr & GC032 \\
\hline \multirow[t]{2}{*}{$\begin{array}{l}\text { Sida rhombifolia } \\
\text { L. }\end{array}$} & Malvaceae & Gorgegit & $S$ & Impotency & $\mathrm{Hu}$ & $\mathrm{R}$ & $F$ & $\mathrm{O}$ & $\begin{array}{l}\text { Drink } \\
\text { concoction } \\
\text { with honey }\end{array}$ & Bo & Spr & GC120 \\
\hline & & & & Wound & $\mathrm{Hu}$ & L & $\mathrm{F}$ & De & Crush and tie & & & \\
\hline \multirow{2}{*}{$\begin{array}{l}\text { Sida tenuicarpa } \\
\text { Vollesen }\end{array}$} & Malvaceae & Chifrig & S & Wound & $\mathrm{Hu}$ & L & $\mathrm{F}$ & $\mathrm{De}$ & Crush and tie & Fwl & Spr & GC153 \\
\hline & & & & $\begin{array}{l}\text { Evil spirit \& } \\
\text { evil eye }\end{array}$ & $\mathrm{Hu}$ & $\mathrm{R}$ & DF & $\begin{array}{l}\text { De } \\
\& O\end{array}$ & $\begin{array}{l}\text { Used as tooth } \\
\text { brush or tie } \\
\text { on neck }\end{array}$ & & & \\
\hline \multirow[t]{2}{*}{$\begin{array}{l}\text { Solanecio gigas } \\
\text { Vatke }\end{array}$} & Asteraceae & Yashikoko gomen & S & Bloating & $\mathrm{Li}$ & L & F & $\mathrm{O}$ & $\begin{array}{l}\text { Pound and } \\
\text { give with } \\
\text { water }\end{array}$ & $\mathrm{Hg}$ & $\mathrm{Pa}$ & GC061 \\
\hline & & & & Evil eye & $\mathrm{Hu}$ & $\mathrm{R}$ & DF & $\begin{array}{l}\mathrm{Na}, \\
\text { O \& } \\
\mathrm{De}\end{array}$ & $\begin{array}{l}\text { Sniff, drink } \\
\text { and fumigate } \\
\text { with } \\
\text { concoction }\end{array}$ & & & \\
\hline $\begin{array}{l}\text { Solanum } \\
\text { anguivi Lam. }\end{array}$ & Solanaceae & Zerch enboy & S & Wound & $\mathrm{Hu}$ & L & DF & De & $\begin{array}{l}\text { Crush, pound } \\
\text { and tie }\end{array}$ & $\mathrm{F}$ & Spr & GC174 \\
\hline
\end{tabular}


Table 4 List of plant species used to treat human and livestock ailments: scientific names, family, vernacular name, growth forms (Gf), Ailments treated, Ailment type(At), parts used (Pu), condition of preparation (Cp), route of administration (Ra), method of preparation, habitat (Ha), distribution(Dn), collection number (Co.No.) in the environ of Tara-gedam and Amba forests (Continued)

\begin{tabular}{|c|c|c|c|c|c|c|c|c|c|c|c|c|}
\hline & & & & Wart & $\mathrm{Hu}$ & $\mathrm{Fr}$ & $\mathrm{F}$ & De & $\begin{array}{l}\text { Cream with } \\
\text { juice }\end{array}$ & & & \\
\hline & & & & $\begin{array}{l}\text { Beating with } \\
\text { stick }\end{array}$ & $\mathrm{Li}$ & $\mathrm{R}$ & $\mathrm{F}$ & $\mathrm{O}$ & $\begin{array}{l}\text { Crush and } \\
\text { give the } \\
\text { infusion }\end{array}$ & & & \\
\hline \multirow[t]{9}{*}{$\begin{array}{l}\text { Solanum } \\
\text { incanum L. }\end{array}$} & \multirow[t]{9}{*}{ Solanaceae } & \multirow[t]{9}{*}{ Yekolla enboy } & \multirow[t]{9}{*}{$S$} & Stomachache & $\mathrm{Hu}$ & $\mathrm{R}$ & $\mathrm{F}$ & O & $\begin{array}{l}\text { Crush, chew } \\
\text { then absorb } \\
\text { juice }\end{array}$ & \multirow[t]{9}{*}{ Fwl } & \multirow[t]{9}{*}{ Spr } & \multirow[t]{9}{*}{ GC059 } \\
\hline & & & & Ring worm & $\mathrm{Hu}$ & $\mathrm{Fr}$ & $\mathrm{F}$ & De & $\begin{array}{l}\text { Heat fruit } \\
\text { then cream } \\
\text { with juice }\end{array}$ & & & \\
\hline & & & & Wart & $\mathrm{Hu}$ & $\mathrm{Fr}$ & $\mathrm{F}$ & De & $\begin{array}{l}\text { Cream with } \\
\text { juice }\end{array}$ & & & \\
\hline & & & & $\begin{array}{l}\text { Arthritis/ } \\
\text { rheumatism }\end{array}$ & $\mathrm{Hu}$ & L & $\mathrm{F}$ & De & $\begin{array}{l}\text { Pound and } \\
\text { tie }\end{array}$ & & & \\
\hline & & & & Leeches & $\mathrm{Li}$ & $\mathrm{Fr}$ & $\mathrm{F}$ & $\mathrm{Na}$ & Insert juice & & & \\
\hline & & & & Diabetic & $\mathrm{Hu}$ & $\mathrm{R}$ & $\mathrm{F}$ & $\mathrm{O}$ & $\begin{array}{l}\text { Chew and } \\
\text { swallow juice }\end{array}$ & & & \\
\hline & & & & Febrile illness & $\mathrm{Li}$ & $\mathrm{R}$ & $\mathrm{F}$ & $\mathrm{O}$ & $\begin{array}{l}\text { Pound and } \\
\text { give with } \\
\text { water }\end{array}$ & & & \\
\hline & & & & Wound & $\mathrm{Hu}$ & $\mathrm{Fr}$ & $\mathrm{F}$ & De & $\begin{array}{l}\text { Cream with } \\
\text { juice }\end{array}$ & & & \\
\hline & & & & $\begin{array}{l}\text { Scorpion } \\
\text { poison }\end{array}$ & $\mathrm{Hu}$ & $\mathrm{Fr}$ & $\mathrm{F}$ & $\mathrm{O}$ & $\begin{array}{l}\text { Drink juice } \\
\text { with water }\end{array}$ & & & \\
\hline $\begin{array}{l}\text { Solanum } \\
\text { marginatum L.f. }\end{array}$ & Solanaceae & Yedega enboy & S & Cough & $\mathrm{Li}$ & $\mathrm{Fr}$ & $\mathrm{F}$ & $\mathrm{Na}$ & $\begin{array}{l}\text { Give juice } \\
\text { with goat } \\
\text { milk }\end{array}$ & $\mathrm{F}$ & $\mathrm{Rr}$ & GC095 \\
\hline \multirow[t]{3}{*}{$\begin{array}{l}\text { Solanum } \\
\text { nigrum L.* }\end{array}$} & \multirow[t]{3}{*}{ Solanaceae } & \multirow[t]{3}{*}{ Awut } & \multirow[t]{3}{*}{$\mathrm{H}$} & Spider poison & $\mathrm{Hu}$ & L & $\mathrm{F}$ & De & $\begin{array}{l}\text { Crush, } \\
\text { squeeze then } \\
\text { cream }\end{array}$ & \multirow[t]{3}{*}{ Fwl } & \multirow[t]{3}{*}{$\mathrm{Rr}$} & \multirow[t]{3}{*}{ GC140 } \\
\hline & & & & Hemorrhoid & $\mathrm{Hu}$ & Ap & DF & De & $\begin{array}{l}\text { Pound and } \\
\text { tie }\end{array}$ & & & \\
\hline & & & & Diarrhea & $\mathrm{Hu}$ & L & $\mathrm{F}$ & O & $\begin{array}{l}\text { Crush, chew } \\
\text { then swallow } \\
\text { juice }\end{array}$ & & & \\
\hline $\begin{array}{l}\text { Steganotaenia } \\
\text { araliacea } \\
\text { Hochst. ex A. } \\
\text { Rich. }\end{array}$ & Apiaceae & Endoka/Yefiyel chew & $\mathrm{T}$ & Hemorrhoid & $\mathrm{Hu}$ & St & DF & $\mathrm{De}$ & $\begin{array}{l}\text { Peel, heat } \\
\text { then apply in } \\
\text { the hot } \\
\text { condition }\end{array}$ & $\mathrm{F}$ & Spr & GC083 \\
\hline \multirow{4}{*}{$\begin{array}{l}\text { Stephania } \\
\text { abyssinica } \\
\text { (Dillon \& A. } \\
\text { Rich.) Walp. }\end{array}$} & \multirow[t]{4}{*}{ Menispermaceae } & \multirow[t]{4}{*}{ Chewchawit } & \multirow[t]{4}{*}{$\mathrm{H}$} & Anthrax & B & $\mathrm{R}$ & F & $\mathrm{O}$ & $\begin{array}{l}\text { Crush and } \\
\text { give with } \\
\text { water }\end{array}$ & \multirow[t]{4}{*}{ Fal } & \multirow[t]{4}{*}{ Spr } & \multirow[t]{4}{*}{ GC121 } \\
\hline & & & & $\begin{array}{l}\text { Anthrax \& } \\
\text { Stomachache }\end{array}$ & $\mathrm{Hu}$ & $\mathrm{R}$ & $\mathrm{F}$ & O & $\begin{array}{l}\text { Chew and } \\
\text { swallow the } \\
\text { juice }\end{array}$ & & & \\
\hline & & & & Rabies & B & $\mathrm{R}$ & $\mathrm{F}$ & $\mathrm{O}$ & $\begin{array}{l}\text { Crushed and } \\
\text { given with } \\
\text { milk and } \\
\text { water }\end{array}$ & & & \\
\hline & & & & Tonsillitis & $\mathrm{Hu}$ & Sh & $F$ & O & $\begin{array}{l}\text { Crush and } \\
\text { drink with } \\
\text { water or } \\
\text { cream on } \\
\text { neck region }\end{array}$ & & & \\
\hline
\end{tabular}


Table 4 List of plant species used to treat human and livestock ailments: scientific names, family, vernacular name, growth forms (Gf), Ailments treated, Ailment type(At), parts used (Pu), condition of preparation (Cp), route of administration (Ra), method of preparation, habitat (Ha), distribution(Dn), collection number (Co.No.) in the environ of Tara-gedam and Amba forests (Continued)

\begin{tabular}{|c|c|c|c|c|c|c|c|c|c|c|c|c|}
\hline \multirow[t]{2}{*}{$\begin{array}{l}\text { Stereospermum } \\
\text { kunthianum } \\
\text { Cham. }\end{array}$} & Bignonaceae & Zana & $\mathrm{T}$ & Eye problem & $\mathrm{Li}$ & B & DF & $\mathrm{O}$ & $\begin{array}{l}\text { Cream the } \\
\text { concoction } \\
\text { with butter } \\
\text { and apply to } \\
\text { cattle }\end{array}$ & $\mathrm{F}$ & Spr & GC017 \\
\hline & & & & $\begin{array}{l}\text { Scorpion \& } \\
\text { Snake poison }\end{array}$ & $\mathrm{Hu}$ & B & $\mathrm{F}$ & De & $\begin{array}{l}\text { Pound and } \\
\text { tie or chew } \\
\text { and swallow } \\
\text { the juice }\end{array}$ & & & \\
\hline $\begin{array}{l}\text { Striga } \\
\text { hermonthica } \\
\text { (Del.) Benth. }\end{array}$ & Scrophularaceae & Gelmit & $\mathrm{H}$ & Bloating & $\mathrm{Li}$ & Ap & DF & $\mathrm{O}$ & $\begin{array}{l}\text { Crush, } \\
\text { powder and } \\
\text { give with } \\
\text { water }\end{array}$ & Fal & Spr & GC144 \\
\hline $\begin{array}{l}\text { Syzygium } \\
\text { guineense } \\
\text { (Willd.) DC. }\end{array}$ & Myrtaceae & Dokima & $\mathrm{T}$ & Diarrhea & $\mathrm{Hu}$ & B & $\mathrm{F}$ & $\mathrm{O}$ & $\begin{array}{l}\text { Crush and } \\
\text { drink with } \\
\text { water }\end{array}$ & Ris & Spr & GC045 \\
\hline \multirow[t]{2}{*}{$\begin{array}{l}\text { Thalictrum } \\
\text { rhynchocarpum } \\
\text { Dill. \& A. Rich. }\end{array}$} & Ranunculaceae & Sire-bizu & $\mathrm{H}$ & $\begin{array}{l}\text { Scrotum } \\
\text { swelling }\end{array}$ & $\mathrm{Hu}$ & $\mathrm{R}$ & $\mathrm{F}$ & De & $\begin{array}{l}\text { Crush and } \\
\text { drink with } \\
\text { TELLA }\end{array}$ & $\mathrm{F}$ & $\mathrm{Rr}$ & GC078 \\
\hline & & & & Impotency & $\mathrm{Hu}$ & $R$ & $\mathrm{~F}$ & $\mathrm{O}$ & $\begin{array}{l}\text { Drink } \\
\text { concoction } \\
\text { with honey }\end{array}$ & & & \\
\hline \multirow[t]{2}{*}{$\begin{array}{l}\text { Tragia brevipes } \\
\text { Pax. }\end{array}$} & Euphorbiaceae & Abelbalit & $\mathrm{H}$ & Swelling & $\mathrm{Hu}$ & $\mathrm{R}$ & $\mathrm{F}$ & De & $\begin{array}{l}\text { Pound and } \\
\text { tie }\end{array}$ & $\mathrm{F}$ & $\mathrm{Rr}$ & GC013 \\
\hline & & & & Impotency & $\mathrm{Hu}$ & $\mathrm{R}$ & $\mathrm{F}$ & $\mathrm{O}$ & $\begin{array}{l}\text { Drink } \\
\text { concoction } \\
\text { with honey }\end{array}$ & & & \\
\hline $\begin{array}{l}\text { Urera } \\
\text { hypselodendron } \\
\text { (Hochst.) ex A. } \\
\text { Rich. }\end{array}$ & Urticaceae & Lankusso & $\mathrm{Cl}$ & Anthrax & $\mathrm{Li}$ & Sh & $\mathrm{F}$ & $\mathrm{O}$ & $\begin{array}{l}\text { Crush and } \\
\text { give with } \\
\text { water }\end{array}$ & $\mathrm{F}$ & Spr & $\begin{array}{l}\text { GC } \\
060\end{array}$ \\
\hline \multirow[t]{2}{*}{$\begin{array}{l}\text { Urtica simensis } \\
\text { Steudel }\end{array}$} & Urticaceae & Sama & $\mathrm{H}$ & Gastric & $\mathrm{Hu}$ & $\mathrm{L}$ & $\mathrm{F}$ & $\mathrm{O}$ & $\begin{array}{l}\text { Roast, grind } \\
\text { and drink } \\
\text { juice }\end{array}$ & $F$ & $\mathrm{Rr}$ & $\begin{array}{l}\text { GC } \\
179\end{array}$ \\
\hline & & & & Wound & $\mathrm{Hu}$ & $L$ & $\mathrm{~F}$ & De & $\begin{array}{l}\text { Grind and } \\
\text { cream with } \\
\text { butter }\end{array}$ & & & \\
\hline \multirow[t]{4}{*}{$\begin{array}{l}\text { Verbasicum } \\
\text { sinaiticum } \\
\text { Benth. }\end{array}$} & Scrophularaceae & Kutitina & S & Stomachache & $\mathrm{Hu}$ & $\mathrm{R}$ & $\mathrm{F}$ & $\mathrm{O}$ & $\begin{array}{l}\text { Pound and } \\
\text { drink with } \\
\text { honey or } \\
\text { water or } \\
\text { butter }\end{array}$ & $\mathrm{F}$ & Spr & GC074 \\
\hline & & & & Diarrhea & $\mathrm{Hu}$ & $\mathrm{R}$ & $\mathrm{F}$ & $\mathrm{O}$ & $\begin{array}{l}\text { Crush and } \\
\text { drink with } \\
\text { water }\end{array}$ & & & \\
\hline & & & & Evil sprit & $\mathrm{Hu}$ & $L$ & $\mathrm{~F}$ & De & $\begin{array}{l}\text { Boil and } \\
\text { fumigate with } \\
\text { the fume }\end{array}$ & & & \\
\hline & & & & Evil eye & $\mathrm{Hu}$ & $\mathrm{R}$ & DF & $\begin{array}{l}\mathrm{Na} \\
\mathrm{O} \& \\
\mathrm{De}\end{array}$ & $\begin{array}{l}\text { Sniff, drink } \\
\text { and fumigate } \\
\text { concoction }\end{array}$ & & & \\
\hline \multirow{2}{*}{$\begin{array}{l}\text { Verbena } \\
\text { officinalis L. }\end{array}$} & Verbenaceae & Atuch & $\mathrm{H}$ & Bleeding & $\mathrm{Hu}$ & $\mathrm{R}$ & $\mathrm{F}$ & De & Crush and tie & Fal & Wy & GC069 \\
\hline & & & & $\begin{array}{l}\text { Evil spirit \& } \\
\text { intestinal } \\
\text { poison }\end{array}$ & $\mathrm{Hu}$ & $\mathrm{Ag}$ & DF & $\mathrm{O}$ & $\begin{array}{l}\text { Crush and } \\
\text { drink with } \\
\text { water }\end{array}$ & & & \\
\hline
\end{tabular}


Table 4 List of plant species used to treat human and livestock ailments: scientific names, family, vernacular name, growth forms (Gf), Ailments treated, Ailment type(At), parts used (Pu), condition of preparation (Cp), route of administration (Ra), method of preparation, habitat (Ha), distribution(Dn), collection number (Co.No.) in the environ of Tara-gedam and Amba forests (Continued)

\begin{tabular}{|c|c|c|c|c|c|c|c|c|c|c|c|c|}
\hline & & & & Evil eye & $\mathrm{Hu}$ & $\mathrm{R}$ & DF & $\begin{array}{l}\mathrm{Na} \\
\& \mathrm{O}\end{array}$ & $\begin{array}{l}\text { Sniff, drink } \\
\text { and fumigate } \\
\text { concoction }\end{array}$ & & & \\
\hline & & & & Tonsillitis & $\mathrm{Hu}$ & Ap & $\mathrm{F}$ & $\mathrm{O}$ & $\begin{array}{l}\text { Crush and } \\
\text { drink with } \\
\text { water }\end{array}$ & & & \\
\hline & & & & Impotency & $\mathrm{Hu}$ & $\mathrm{R}$ & D & $\mathrm{O}$ & $\begin{array}{l}\text { Drink } \\
\text { concoction } \\
\text { with honey }\end{array}$ & & & \\
\hline & & & & Deafness & $\mathrm{Hu}$ & $\mathrm{L}$ & $\mathrm{F}$ & Et & $\begin{array}{l}\text { Pound and } \\
\text { ingest juice } \\
\text { with water }\end{array}$ & & & \\
\hline & & & & $\begin{array}{l}\text { Stomachache } \\
\text { \& Anthrax }\end{array}$ & $\mathrm{Hu}$ & $\mathrm{R}$ & $\mathrm{F}$ & $\mathrm{O}$ & $\begin{array}{l}\text { Chew and } \\
\text { swallow the } \\
\text { juice }\end{array}$ & & & \\
\hline \multirow[t]{2}{*}{$\begin{array}{l}\text { Vernonia } \\
\text { adoensis Sch. } \\
\text { Bip ex Walp. }\end{array}$} & Asteraceae & Eras abera/ Este musaye & $\mathrm{S}$ & $\begin{array}{l}\text { LIKFIT (skin } \\
\text { rash) }\end{array}$ & $\mathrm{Hu}$ & $\mathrm{R}$ & $\mathrm{F}$ & De & $\begin{array}{l}\text { Crush. } \\
\text { powder then } \\
\text { cream with } \\
\text { butter }\end{array}$ & $\mathrm{Hg}$ & Spr & GC147 \\
\hline & & & & $\begin{array}{l}\text { Amoeba, } \\
\text { Gardiasis, } \\
\text { Gastric \& } \\
\text { Snake poison }\end{array}$ & $\mathrm{Hu}$ & $\mathrm{R}$ & $\mathrm{F}$ & $\mathrm{O}$ & $\begin{array}{l}\text { Crush, } \\
\text { powder then } \\
\text { drink with } \\
\text { water or } \\
\text { Chew and } \\
\text { swallow juice }\end{array}$ & & & \\
\hline \multirow[t]{3}{*}{$\begin{array}{l}\text { Vernonia } \\
\text { amygdalina } \\
\text { Del. }\end{array}$} & Asteraceae & Girawa & $S$ & Bloating & $\mathrm{Li}$ & $\mathrm{L}$ & $\mathrm{F}$ & $\mathrm{O}$ & $\begin{array}{l}\text { Crush and } \\
\text { give with } \\
\text { water }\end{array}$ & $\mathrm{Hg}$ & $\operatorname{Rr}$ & GC055 \\
\hline & & & & Dandruff & $\mathrm{Hu}$ & $\mathrm{L}$ & $\mathrm{F}$ & De & $\begin{array}{l}\text { Pound and } \\
\text { cream }\end{array}$ & & & \\
\hline & & & & Impotency & $\mathrm{Hu}$ & $\mathrm{R}$ & $\mathrm{F}$ & $\mathrm{O}$ & $\begin{array}{l}\text { Drink the } \\
\text { concoction } \\
\text { with TELLA }\end{array}$ & & & \\
\hline $\begin{array}{l}\text { Vernonia } \\
\text { myriantha } \\
\text { Hook.f. }\end{array}$ & Asteraceae & Kotkoto & $S$ & Impotency & $\mathrm{Hu}$ & $\mathrm{R}$ & DF & $\mathrm{O}$ & $\begin{array}{l}\text { Drink the } \\
\text { concoction } \\
\text { with TELLA }\end{array}$ & Fwl & Wy & GC057 \\
\hline Vicia faba $\mathrm{L}$. & Fabaceae & Bakela & $\mathrm{H}$ & Anemia & $\mathrm{Hu}$ & $\mathrm{Sd}$ & D & $\mathrm{O}$ & $\begin{array}{l}\text { Roast and } \\
\text { drink infusion }\end{array}$ & $\mathrm{Hg}$ & Spr & GC109 \\
\hline \multirow[t]{4}{*}{$\begin{array}{l}\text { Withania } \\
\text { somnifera (L.) } \\
\text { Dunal in DC. }\end{array}$} & Solanaceae & Giziewa & $S$ & $\begin{array}{l}\text { Evil eye \& evil } \\
\text { sprit }\end{array}$ & $\mathrm{Hu}$ & $\begin{array}{l}L \& \\
R\end{array}$ & DF & $\mathrm{O}$ & $\begin{array}{l}\text { Crush and } \\
\text { drink with } \\
\text { water or } \\
\text { fumigate with } \\
\text { the fume }\end{array}$ & $\mathrm{Hg}$ & $\mathrm{Rr}$ & GC048 \\
\hline & & & & $\begin{array}{l}\text { Tape worm \& } \\
\text { Babies disease }\end{array}$ & $\mathrm{Hu}$ & L & DF & $\mathrm{De}$ & $\begin{array}{l}\text { Fumigate in a } \\
\text { closed } \\
\text { fashion }\end{array}$ & & & \\
\hline & & & & Cough & $\mathrm{Hu}$ & L & $\mathrm{F}$ & $\mathrm{O}$ & $\begin{array}{l}\text { Crush and } \\
\text { boil with milk } \\
\text { then drink }\end{array}$ & & & \\
\hline & & & & Impotency & $\mathrm{Hu}$ & $\mathrm{R}$ & $\mathrm{F}$ & $\mathrm{O}$ & $\begin{array}{l}\text { Drink } \\
\text { concoction } \\
\text { with honey }\end{array}$ & & & \\
\hline $\begin{array}{l}\text { Xanthium } \\
\text { strumarium L. }\end{array}$ & Asteraceae & Gid zemede & $\mathrm{H}$ & Dandruff & $\mathrm{Hu}$ & L & $\mathrm{F}$ & De & $\begin{array}{l}\text { Rub, squeeze } \\
\text { then cream }\end{array}$ & Fwl & Spr & GC112 \\
\hline $\begin{array}{l}\text { Ximenia } \\
\text { americana L.* }\end{array}$ & Olacaceae & Enkoy & $S$ & Wound & $\mathrm{Hu}$ & B & DF & De & $\begin{array}{l}\text { Crush, grind } \\
\text { and cream }\end{array}$ & $\mathrm{F}$ & $\mathrm{Rr}$ & GC054 \\
\hline
\end{tabular}


Table 4 List of plant species used to treat human and livestock ailments: scientific names, family, vernacular name, growth forms (Gf), Ailments treated, Ailment type(At), parts used (Pu), condition of preparation (Cp), route of administration $(\mathrm{Ra})$, method of preparation, habitat $(\mathrm{Ha})$, distribution( $\mathrm{Dn})$, collection number (Co.No.) in the environ of Tara-gedam and Amba forests (Continued)

\begin{tabular}{|c|c|c|c|c|c|c|c|c|c|c|c|c|}
\hline Zea mays L. & Poaceae & Mashilla & $\mathrm{H}$ & Dandruff & $\mathrm{Hu}$ & Sw & $\mathrm{F}$ & De & $\begin{array}{l}\text { Burn and } \\
\text { cream ashes } \\
\text { with butter }\end{array}$ & $\mathrm{Hg}$ & Wy & GC030 \\
\hline \multirow{4}{*}{$\begin{array}{l}\text { Zehneria scabra } \\
\text { (Linn. f.) Sond. }\end{array}$} & \multirow[t]{4}{*}{ Cucurbitaceae } & \multirow[t]{4}{*}{ Hareg resa } & \multirow[t]{4}{*}{$\mathrm{Cl}$} & Swelling & $\mathrm{Hu}$ & L & $\mathrm{F}$ & De & Crush and tie & Ah & $\operatorname{Rr}$ & GC149 \\
\hline & & & & Wound & $\mathrm{Li}$ & $\mathrm{Ag}$ & $\mathrm{F}$ & De & $\begin{array}{l}\text { Rub and } \\
\text { cream }\end{array}$ & & & \\
\hline & & & & Febrile illness & $\mathrm{Hu}$ & $\mathrm{Ag}$ & $\mathrm{F}$ & De & $\begin{array}{l}\text { Boil and take } \\
\text { the fume in } \\
\text { enclosed } \\
\text { fashion }\end{array}$ & & & \\
\hline & & & & Diarrhea & $\mathrm{Hu}$ & L & $\mathrm{F}$ & $\mathrm{O}$ & $\begin{array}{l}\text { Crush, chew } \\
\text { then swallow } \\
\text { juice }\end{array}$ & & & \\
\hline $\begin{array}{l}\text { Ziziphus spina- } \\
\text { christi (L.) Desf. } \\
*\end{array}$ & Rhamnaceae & Gava & $\mathrm{T}$ & Dandruff & $\mathrm{Hu}$ & $\mathrm{L}$ & $\mathrm{F}$ & De & $\begin{array}{l}\text { Pound and } \\
\text { cream }\end{array}$ & $\mathrm{Hg}$ & $\mathrm{Pa}$ & GC163 \\
\hline
\end{tabular}

Key: Parts Used: B (stem bark), Rb (root bark), R (root), L (leaf), Ap (all part), St (stem), Bu (bulb), Lx (latex), Fl (flower), Sd (Seed), Sh (shoot), Fr (fruit), Sp (Sap), Sw (Straw), Gm ( gum), Ag (above ground); Growth forms (Gf):-S (shrub), T (tree), Cl (climber), H (herb), P (parasite); Ailment type (At): Hu (human) LI (livestock); CP (condition of preparation): $\mathrm{F}$ (fresh), D (dry), DF/FD (dry and fresh); Route of administration (Ra): De (Dermal), O (Oral), Na (nasal), Op (Optical), Va (Vaginal), Et (Ear tube); Habitat (Ha): Wild (F (Fores), Fal (Farm land), Fwl (Fallow land), Rs (Road side), Ris (river side), Ah (around home)), Aw (All wild type of habitats i.e Forest, Farm land, Fallow land, Road side, river side and around home), Bo (all wild type habitats and homegarden), Hg (Homegarden), Distribution(Dn): Spr (Sparse), Wy (Widely), Rr (Rare), Pa (Particular area); Co. No.(Collection number) *Wild food plant species.

accessed easily, the previously stored remedy would be buried in the ground for one day (from the eve of the end of the first day of the New Year), after which time it is declared safe to be used. It was explained that remedies were stored secretly in a very secure place (mostly outside the living house at the top of the wall to keep them far from children) and no one is allowed to touch them without permission.

\section{Route of remedy administration and dosage}

\section{determination}

It was found that the local people employ about 10 ways of medicine administration routes with varying frequencies. Of the total, 157 (44.9\%) prescriptions were mainly those said to be applied through oral route (Table 14). The dosage varied between age and patient's capacity as judged by healers. Traditional ways of dosage determination included measurements, namely, ATQ (referring to the size of the finger stripe/line, mostly of the small finger), TFIR (referring to the size of a fingernail), FINJAL (referring to the volume of the coffee cup), BIRCHIKO (referring to the volume of a glass, mostly of tea glass). And TASSA (referring to the volume of a tin can), MANKIA (referring to the size of a teaspoon) and FAGA (referring to a container made from a small fruit of the bottle gourd (Lagenaria siceraria) as well as number (leaves, fruits, seeds), size and droplets of plant parts. Smaller sizes (ATQ and TFIR) were used to determine dosages of the most toxic plants including Euphorbia abyssinica, Stephania abyssinica and Calpurnia aurea, and the two measurements plus FINJAL, BIRCHIKO and MANKIA are meant for oral administration of medicine for the treatment of internal human ailments. FINJAL, BIRCHIKO, TASSA and FAGA were used for less toxic plants that were diluted with liquid additives including tea, milk, coffee and water. Remedies were mixed mostly with water, honey, tea, milk, coffee, and dosages prescribed as half, one, two, and so on of materials used per day based on the nature of plants and patient's age and general condition (body, health). TASSA and FAGA were prescribed for use to treat livestock ailments while FAGA for preparation and dosage determination for external application of remedies in the cases of both humans and livestock treatment. The concepts of dosage and measurement do exist in the traditional herbal medical system of the community as it emerges from the practices albeit the low precision. Even though the experienced medicinal plant practitioners showed serious concerns in determining the dosages very carefully; the measuring devices they used do not allow delivery of precise amounts. The members of the association of healers and some other local community members reported the effectiveness of traditional medicine, but they expressed discomfort when it comes to the amount given particularly in the case of internal human medicines. They actually recommended that technical assistance and psychological support through training must be given to minimize the fear and effect of incompatible dosage of remedies on patients. The measurements used to determine the dosages are not standardized except categorization by age, physical appearance and health conditions. The absence of adverse effects of traditional herbal medicines after administration was most 
frequently mentioned by the traditional healers. Coffee and milk were mentioned for use as antidotes when formulations were made from Euphorbia abyssinica, for malaria, and Calpurnia aurea for diarrhea and anesthesia. Likewise, local beer (TELLA) is used as antidote when Asparagus africanus is used to treat impotence. The traditional healers indicated that they use the antidotes for dilution in cases of adverse effects.

\section{Marketed medicinal plants in the study area}

Survey of two towns in the proximity of the study sites (Addis Zemen and Yifag) did not show any medicinal plant mentioned during the interviews presented on the market. The respondents explained that most healers prepared and sold traditional medicinal plants in the home rather than in the open market. Healers usually had big signposts in front of their homes listing the health problems they treat. Some medicinal plants were marketed mainly for other use values (spices and food) but once bought they could be used as medicine at home as part of the common family home treatment. These include Allium sativum, Ruta chalepensis, Brassica carinata and Cicer arietinum usually traded for use as edible spices. On the other hand, Carica papaya, Citrus aurantifolia, Citrus aurantium, Coffea arabica, Cucurbita pepo, Linum usitatissimum, Mimusops kummel, Persea americana, Prunus persica, Punica granatum, Zea mays, Eragrostis tef, Capsicum annuum and Vicia faba were bought from the market for use as food items.

\section{Taboos connected with handling and use of medicinal plants}

Some of the taboos reported by experienced medicinal plant experts concern times of collection, ways of collection, preparation materials, administration and storage. Most of the medicinal plants were said to be collected on Wednesdays and Fridays in the early morning hours without contact and without talking to any other person and this is related to healers' beliefs that doing it otherwise would reduce the efficacy of the herbal medicine. In the preparation of a single remedy, plant parts are mostly taken from individuals of the same species growing in three or seven different places. One healer said that this increases its remedial effectiveness. This could be a way of balancing the amount of phytochemical and pharmacological constituents based on habitat variation. Collection materials are KARA (kind of knife), ANKASIE/TORE (metallic spear), WEYRA EJETA MEKOFERIA (digger with handle made of Olea europaea ssp. cuspidata wood) and most of the time stationary stones are the preferred preparation places. It was mentioned that sexual intercourse is forbidden for healers and patients alike during any medicinal plant collection, preparation and application.

\section{Variation of indigenous plant knowledge in the study area}

Significant correlation (Spearman correlation test, $r=-0.450, \alpha=0.05, p=0.046)$ was observed between male and female informants on the number of medicinal plant species they knew. The test, however, did not indicate significant correlation between healers and general informants (Spearman correlation test, $r=-0.002, \alpha=0.05, p=0.991)$ regarding the number of medicinal plant species they reported. The comparison of knowledge and experience of age groups (35-50 and 51-84) showed significant differences $(\mathrm{P}<0.05)$ while there was no significant difference between age groups 19-34 and 35-50 considering plant names and the respective medicinal uses $(t=0.05$, two tailed and $\mathrm{df}=52$ ). Progressively increasing results were obtained with increasing age of informants (Figure 3).

Local community members in Washa Indiras, Kidanemhret and Kualla Yihuans gave 162, 95 and 91 medicinal plant names with 128, 95 and 86 medicinal uses respectively. Informants from Washa Indiras village reported the highest plant names (162) and uses (128), while those in Yifag Akababi and Asiba turned in the least numbers $(58,56)$ and uses $(52,50)$ respectively. However, not all communities living nearby the forests gave higher reports compared to distant villages. For example, Tibabosgie is the nearest village to the forest, but the report from informants showed relatively lower names (48) and uses (50) than the other villages found relatively far from the forest, namely Abuarra (92 names and 80 uses), Lomye (73 names and 80 uses) and Agamoch (69 names and 66 uses). On the other hand, Mantogera village is located nearest to the forest, but the results showed 61 names and 61 uses, which is less than other nearby villages in the same (woina dega) agroclimatic zone. Furthermore, in Aguat Mafsesha located at higher altitude of all villages found in dega agroclimatic zone, showed that informants could only recall a few species and uses (40 names and 43 uses). Generally, however, informants in villages near the forest knew more plants (38.5\%) and uses (38.9\%) than those located in towns $(30.3 \%, 30.0 \%)$ and far away from forests $(30.8 \%, 31.5 \%)$.

\section{Indigenous medicinal plant knowledge development and sharing}

Traditional knowledge of medicinal plants in most cases is passed along the family line from parents and other intimates, especially gifted family members (which they described as EJU YEMISEMRLET, meaning one whose hands are skillful and effectual). Some of the traditional knowledge is generated through the community by listening and practicing while some copied secretly and systematically by following and observing the 
Table 5 The six most acclaimed medicinal plants based on informant citation

\begin{tabular}{|c|c|c|c|c|}
\hline Scientific name & Ailments claimed to treat & No. of citations & Percentage & Rank \\
\hline Zehneria scabra & Diarrhea, wound, febrile illness and swelling & 60 & 57.14 & $1^{\text {st }}$ \\
\hline Stephania abyssinica & Human and livestock anthrax, tonsillitis, rabies and stomachache & 55 & 52.40 & $2^{\text {nd }}$ \\
\hline Otostegia integrifolia & Stomachache, hen's coccolida, epidemic diseases and common cold & 40 & 38.10 & $3^{\text {rd }}$ \\
\hline Verbascum sinaiticum & Stomachache, diarrhea, evil eye \& evil sprit & 32 & 30.47 & $4^{\text {th }}$ \\
\hline Capparis tomentosa & Evil eye, and epidemic diseases & 27 & 25.71 & $5^{\text {th }}$ \\
\hline Achyranthes aspera & $\begin{array}{l}\text { Tape worm, wounds, excessive menstrual flow, tonsillitis, } \\
\text { bleeding, bone fracture, and eye problems }\end{array}$ & 25 & 23.80 & $6^{\text {th }}$ \\
\hline
\end{tabular}

knowledgeable individuals at times of medicinal plant collection and preparation. Others develop and transfer their medicinal plant knowledge to generations by following up healers after seeking treatment of their family members. In very few cases, individuals developed their medicinal plant knowledge upon careful observation of domestic carnivores, especially the cat, which immediately consumes medicinal plant parts upon preying on poisonous snakes, scorpions and spiders. One healer reported his discovery in this way of Vernonia adoensis for the treatment of snake poison. Medicinal plant experts have developed some traditional medicinal plant knowledge from observations of animal feeding to know the plants that are never consumed, which hints at plants not for internal use to ensure safety of the vital organs but rather used for the treatment of dermal ailments such as wounds because of their possible toxic nature. Furthermore, experienced medicinal plant experts create new medicinal knowledge by relating the plant odour with previously known medicinal plants. Some healers were seen recording ethnomedicinal knowledge in small notebooks during fieldwork, which may testify their curiosity and keenness to develop and transfer indigenous knowledge to the next generation.

\section{Threats to and conservation of medicinal plants and associated indigenous knowledge}

The study found that medicinal plants are faced with threats in their habitats. Informants claimed that long before the past ten to twenty years Tara-gedam and the surrounding areas were full of natural vegetation around the farmlands, riversides and grazing lands in addition to the wealth of plant species in number and diversity in the forests. They further asserted that in those days almost all the medicinal plants were easily accessible within short distances of the living place. Today, it is not an easy task to get medicinal plants out of Tara-gedam and Amba forests due to habitat modification. Most informants perceived that agricultural expansion was the main threat to medicinal plants, firewood collection the next and others follow (Table 15). Similarly, preference ranking of five most threatened medicinal plant species indicated that Withania somnifera and Huernia macrocarpa are the two most threatened medicinal plants (Table 16). Through further discussion and interview with informants, 63 plant species that were said to have become sparse in distribution were recorded along with five species restricted in occurrence and in most cases found in the homegardens in recent years (Figure 4).

Conservation efforts specifically targeted to medicinal plants do not exist in the District. However, some of the medicinal plants are raised in the governmental nurseries for other purposes and conserved in the protected governmental and Orthodox Tewahedo church forests. The well known Tara-gedam and Amba natural forests and other relatively smaller patches of vegetation and plantations found in each kebele are nowadays being protected by the local people living around the forest fringes in collaboration with the government. Some of the medicinal plants occurring in the Orthodox Tewahedo church forests were Adiantum capillus-veneris, Clerodendrum myricoides, Juniperus procera, Millettia ferruginea, Schefflera abyssinica, Urera hypselodendron and Ziziphus spina-christi. The informants elaborated that some of the medicinal plants collected from the

Table 6 Single medicinal plant species prescribed for treatment of higher number of ailments

\begin{tabular}{|c|c|c|c|}
\hline Plant species name & No. of ailment treated & Plant species name & No. of ailment treated \\
\hline Justicia schimperiana & 11 & $\begin{array}{c}\text { Achyranthes aspera, Cucumis ficifolius } \\
\text { and Euphorbia abyssinica }\end{array}$ & 7 each \\
\hline $\begin{array}{l}\text { Croton macrostachyus, Verbena officinalis } \\
\text { and Solanum incanum }\end{array}$ & 9 each & $\begin{array}{c}\text { Ferula communis, Cynoglossum, coeruleum, } \\
\text { Asparagus africanus, Calpurnia aurea }\end{array}$ & 6 each \\
\hline Phytolacca dodecandra & 8 & & \\
\hline
\end{tabular}




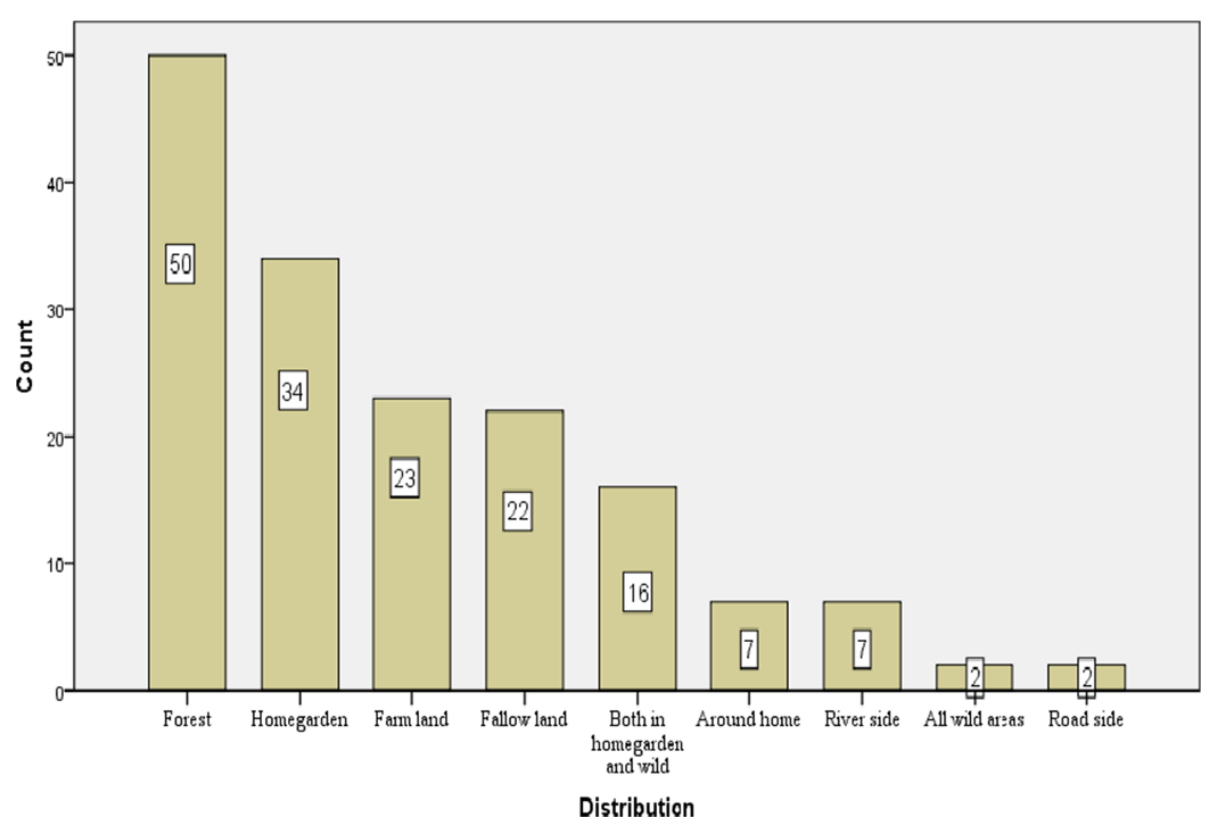

Figure 2 Distribution of medicinal plant species in different habitats.

homegardens namely, Persea americana, Citrus aurantifolia, Citrus aurantium, Coffea arabica, Cordia africana, Ficus sur, Schinus molle and Punica granatum were those raised from seedlings taken from the nursery. It was also observed that the local farmers make use of their indigenous knowledge in protecting important plant species on their farmlands, homegardens, or as live fence. Few traditional healers cultivate very rare species in their homegardens. Healers mentioned the difficulty of cultivating species that cannot be propagated outside their natural habitats and that they have to travel long distances for several hours to get the needed medicinal plants. Alternatively, healers may choose to get (on appointed date) such plants upon cash payment for people who are living in the vicinity of the medicinal plants. Medicinal plants that are known to have additional uses (ornamentals, fuel, forage, spice, food and soil conservation) in the area were planted most frequently in homegardens and farmlands. Allium sativum, Foeniculum vulgare, Lepidium sativum, Ocimum gratissimum, Ruta chalepensis, Schinus molle and few others were commonly planted.

Furthermore, the District administration has started considering the indigenous knowledge of the people as testified by the priority given to establish traditional health practitioners association along with the provision of some technical training and discussion on biodiversity conservation concepts. A good justification for the above scenario is the observation during our field study in the area the mutual exchange of knowledge and remedies at the time of monthly meetings. The first author had a chance to participate in two of their meetings and was kindly given permission to record the information.

\section{Discussion}

Despite the efforts made, only few women could take part in the study partly because of the tradition and being the usual case when the interviewers are men as in our case. Women are generally not expected to appear in public or discuss with stranger men both by society

Table 7 The most common disease with their respective number of medicinal plant species

\begin{tabular}{cccccc}
\hline S.no & Ailments & No of species for each ailment & S.no & Ailments & No of species for each ailment \\
\hline 1 & Wound & 42 & 6 & Impotence & 11 \\
2 & Stomachache & 25 & 7 & $\begin{array}{c}\text { Tonsillitis, rabies, hemorrhoid, } \\
\text { fibril illness, and snake bite }\end{array}$ & 10 \\
3 & Intestinal parasites & 23 & 8 & Dandruff & 8 \\
4 & Anthrax & 16 & 9 & Livestock bloating and malaria & Common cold and cough \\
5 & Diarrhea & 13 & & & 6 \\
\hline
\end{tabular}


Table 8 ICF value for each disease category

\begin{tabular}{lcc}
\hline Disease categories & Nu & Nur \\
\hline Livestock diseases (external parasites, beating with stick and sun stroke) & 16 & 94 \\
Febrile illness, headache, anemia, brain tension and malaria & 19 & 0.84 \\
Rabies & 11 & 80 \\
Gastrointestinal disorders & 52 & 46 \\
Dermal diseases (wound and skin diseases) & 72 & 205 \\
Bone fracture and Arthritis & 7 & 221 \\
Reproductive and sexual organs & 22 & 18 \\
Bleeding and hypertension & 7 & 0.78 \\
Respiratory diseases (asthmatic reactions, cough, common cold, leech and tonsillitis) & 24 & 0.75 \\
Sense organs like eye and ear problems & 21 & 14 \\
Spider, snake, and scorpion poisons and bites & 18 & 48 \\
General disease (tension, epidemic, baby diseases and undefined diseases) & 0.65 \\
Organ diseases (diabetes, heart problem, jaundice, kidney infection, pneumonia, urinary problem) & 28 & 32 \\
Anthrax, cancer and hemorrhoid & 12 & 0.54 \\
\hline
\end{tabular}

and family (husbands deny permission in most cases) or other socio-cultural reasons, which our female informants refrained from describing openly. There were very few women practitioners in the community. More informants are expected to yield more knowledge of plants procured from the wild as was reported by other researchers [33-35]. The rich ethnoecological knowledge was revealed in their elaboration and categorization of the ecological units. They recognized six landscape, five soil and five vegetation types, reflecting their deep understanding of the differences and similarities in these key environmental components. This emanates from the ethnobotanical/ethnoecological knowledge that was shaped over generations and which they use for describing, managing and utilizing the land, the soil and vegetation. Their knowledge also stretches to the individual plants which they grouped into use categories, morphological classes and adaptive forms. Soils which were identified based on colour and texture are applied to determine and select those suitable for the type of crop varieties to be grown on a specific land. This knowledge

Table 9 Simple preference ranking of six medicinal plants used against wound in the study area

\begin{tabular}{lccccccccc}
\hline Medicinal plant species & \multicolumn{8}{c}{ Respondents (R1-R7) } \\
\cline { 2 - 10 } & $\mathbf{R}_{\mathbf{1}}$ & $\mathbf{R}_{\mathbf{2}}$ & $\mathbf{R}_{\mathbf{3}}$ & $\mathbf{R}_{\mathbf{4}}$ & $\mathbf{R}_{\mathbf{5}}$ & $\mathbf{R}_{\mathbf{6}}$ & $\mathbf{R}_{\mathbf{7}}$ & Total & Rank \\
\hline Brucea antidysenterica & 5 & 5 & 1 & 4 & 6 & 5 & 3 & 29 & $3^{\text {rd }}$ \\
Cordia africana & 6 & 6 & 5 & 5 & 5 & 6 & 6 & 39 & $1^{\text {st }}$ \\
Dodonaea angustifolia & 3 & 2 & 6 & 1 & 4 & 3 & 1 & 20 & $4^{\text {th }}$ \\
Ficus carica & 2 & 1 & 3 & 3 & 1 & 2 & 2 & 14 & $6^{\text {th }}$ \\
Plantago lanceolata & 1 & 3 & 2 & 2 & 2 & 1 & 4 & 15 & $5^{\text {th }}$ \\
Sida rhombifolia & 4 & 4 & 4 & 6 & 3 & 4 & 5 & 30 & $2^{\text {nd }}$ \\
\hline
\end{tabular}

shares similarities with the modern classification system [36] and the system used in another part of Ethiopia [37]. Such broad-based indigenous knowledge systems are indicative of prolonged experience, relationship and interaction of people with the biotic and abiotic components of the environment as rightly described for other areas in Ethiopia [38-40].

The top three families (Asteraceae, Fabaceae and Solanaceae) reported in this study are among those represented with higher number of taxa in the Ethiopian flora [39-44] and also found to have higher number of medicinal plants by other researchers working in other parts of Ethiopia [45-47]. This might be related to possession of more species that are widely distributed in almost all ecological areas and habitats since the Fabaceae and Asteraceae are respectively the first and third largest families of angiosperms in the Ethiopian flora [48]. These two families have many uses for the community as reported by other researchers [44-46,49]. The diversity of genera and families (29 with $2-14$ species in many genera) is a good indication for the study area

Table 10 Paired comparison on five medicinal plants used to treat stomachache in the study area

\begin{tabular}{lccccccccc}
\hline Medicinal plants used & \multicolumn{8}{c}{ Respondents (R1-R7) } \\
\cline { 2 - 10 } & $\mathbf{R}_{\mathbf{1}}$ & $\mathbf{R}_{\mathbf{2}}$ & $\mathbf{R}_{\mathbf{3}}$ & $\mathbf{R}_{\mathbf{4}}$ & $\mathbf{R}_{\mathbf{5}}$ & $\mathbf{R}_{\mathbf{6}}$ & $\mathbf{R}_{\mathbf{7}}$ & Total & $\mathbf{R a n k}$ \\
\hline Cucumis ficifolius & 1 & 2 & 1 & 2 & 2 & 2 & 1 & 11 & $4^{\text {th }}$ \\
Indigofera prieureana & 2 & 2 & 3 & 2 & 3 & 3 & 2 & 17 & $2^{\text {nd }}$ \\
Otostegia integrifolia & 0 & 1 & 0 & 2 & 1 & 1 & 3 & 8 & $5^{\text {th }}$ \\
Stephania abyssinica & 4 & 4 & 4 & 4 & 1 & 3 & 2 & 22 & $1^{\text {st }}$ \\
Verbascum sinaiticum & 3 & 1 & 2 & 0 & 3 & 1 & 2 & 12 & $3^{\text {rd }}$ \\
\hline
\end{tabular}


Table 11 Matrix ranks of six multipurpose medicinal plants in the study area

\begin{tabular}{|c|c|c|c|c|c|c|c|c|c|}
\hline Plant species name & Medicine & Cash income & Fuelwood & Food & Forage/fodder & Construction/building & Shade & Total & Rank \\
\hline Carissa spinarum & 5 & 4 & 5 & 4 & 4 & 2 & 1 & 25 & $2^{\text {nd }}$ \\
\hline Cordia africana & 4 & 5 & 3 & 5 & 5 & 2 & 3 & 27 & $1^{\text {st }}$ \\
\hline Croton macrostachyus & 4 & 1 & 2 & 0 & 1 & 2 & 5 & 15 & $6^{\text {th }}$ \\
\hline Ficus sur & 2 & 3 & 2 & 5 & 4 & 2 & 5 & 23 & $4^{\text {th }}$ \\
\hline Mimusops kummel & 2 & 4 & 1 & 5 & 2 & 2 & 5 & 21 & $5^{\text {th }}$ \\
\hline Olea europaea ssp. cuspidata & 3 & 5 & 5 & 0 & 4 & 5 & 2 & 24 & $3^{\text {rd }}$ \\
\hline
\end{tabular}

being an important reservoir of medicinal plants and ethnomedicinal knowledge. Dependence on a great diversity of plant species for treatment of ailments is a good indicator of profound knowledge on medicinal plants. The six most cited medicinal plants that have relatively higher percentages of informants' consensus could be considered for further analyses. The fact that Achyranthes aspera came both in the most cited and most effective medicinal plants for treating different diseases may indicate that in the long term this species could be locally threatened due to overharvesting. At the time of field data collection, the species was found widely distributed in both the wild lands as well as in and around homegardens.

Eight to fifty-five medicinal plant species recorded in this study have also been documented as medicinal in other parts of Ethiopia as our review of 20 sources $[34,39-44,46,49-60]$ showed. This analysis confirms that those medicinal plants are important in the healthcare systems of different cultures in Ethiopia. On the other hand, 31 of the medicinal plant species reported in our study have not been mention in any of the ethnobotanical literature sources reviewed [34,39-44,46,49-60] suggesting that while the knowledge is shared in some respects it also has some uniqueness to the study communities.

The finding that shrubs and herbs were the most abundant medicinal plants indicated that people rely more on such plants, which may relate to the fact that they are relatively common compared to other growth forms. Other researchers $[41,47,53,59,61]$ also found that shrubs and herbs are the most frequent medicinal plant species. Most of the wild medicinal plants were accessed from Tara-gedam and Amba forests. Healers and some knowledgeable members of the local community were seen cultivating some medicinal plants in their homegardens for easy access and use of fresh parts at times of remedy preparation. The distribution of medicinal plants in the wild, homegardens and in both premises [39-41,62,58] as well as finding of more species in the wild environments were reported by other researchers $[33,43,47,62]$ in Ethiopia and other countries $[63,64]$.

Use of diverse plant species in the treatment of ailments implied that the people of the study area to date prevent and cure human and livestock ailments with plant materials collected from the surrounding areas. Less number of livestock diseases and medicinal plants were reported compared to those of humans, which could probably be due to the fact that the people give more attention to human ailments compared to livestock diseases. Generally, the local people affirmed that they first try to find medicines for human ailments and then search for remedies for livestock ailments as reported in other areas [38]. The healers also mentioned that they refer to the pharmacopeias (ancient herbals written on parchment) to learn about medicinal plants and treatments for human diseases. Traditional pharmacopeias have also helped to transfer the knowledge to more people. Treatment of human ailments like womb problem, sterility of females, prolonging the life of embryos in the uterus, expelling foreign particles from the eyes and ears, and livestock ailments like increasing sexual

Table 12 Frequency of plant parts used for the preparation of remedies

\begin{tabular}{|c|c|c|c|c|c|c|c|}
\hline $\begin{array}{l}\text { Plant parts } \\
\text { used }\end{array}$ & No. of preparations & Percentage & No. of species & Plant parts used & No. of preparations each & Percentage & No. of species \\
\hline Leaf & 109 & 31.2 & 56 & Stem & 6 & 1.7 & 4 \\
\hline Root & 108 & 30.9 & 45 & Bulb & 5 & 1.4 & 1 \\
\hline Fruit & 25 & 7.2 & 13 & Flower & 4 & 1.1 & 3 \\
\hline Bark & 15 & 4.3 & 11 & Sap & 1 & 0.3 & 1 \\
\hline Shoot & 15 & 4.3 & 5 & Gum & 1 & 0.3 & 1 \\
\hline Latex & 13 & 3.7 & 6 & All parts & 10 & 2.9 & 7 \\
\hline Seed & 7 & 2.0 & 5 & Two and three parts & 15 & 4.3 & 13 \\
\hline
\end{tabular}


Table 13 Mode of preparation of medicinal plants

\begin{tabular}{lcc}
\hline Types of preparation & $\begin{array}{c}\text { Frequency of } \\
\text { preparation }\end{array}$ & Percentages \\
\hline Crushing & 118 & 35.01 \\
$\begin{array}{l}\text { Grinding, concoction } \\
\text { and creaming }\end{array}$ & 50 & 14.80 \\
$\begin{array}{l}\text { Boiling, heating, burning } \\
\text { and fumigation }\end{array}$ & 49 & 14.50 \\
$\begin{array}{l}\text { Chewing, spitting and } \\
\text { absorbing fluid/juice }\end{array}$ & 32 & 9.50 \\
Rubbing and squeezing & 24 & 7.10 \\
Using unprocessed plant part & 23 & 6.80 \\
Pounding and making infusion & 23 & 6.80 \\
Chopping and breaking & 18 & 5.30 \\
\hline
\end{tabular}

needs and beating with stick are new plant uses not encountered in any of the previous publications reviewed.

Higher ICF values as in external parasites, beating with stick and sun stroke in the case of livestock, and febrile illness, headache, anemia, brain tension and malaria in human being are indicative of the presence of similar ethnomedicinal plant knowledge and their continued usage in similar ways among community members $[32,64]$ as also reported from other parts of Ethiopia [39-41].

Cordia africana, the most multipurpose species as in other areas [62], would be imagined to be most threatened in the future. The clue to this is its rare occurrence with sparse distribution around farmlands and some homegardens. This scarcity was due to over harvesting not only for medicinal purpose, but also for other uses, notably for timber production. All of the medicinal plant species and the top ranking ones in particular need urgent conservation actions and adoption of a suitable system of sustainable use.

Table 14 Mode of administration of the plant remedies

\begin{tabular}{lcc}
\hline $\begin{array}{l}\text { Mode of } \\
\text { administration }\end{array}$ & $\begin{array}{c}\text { Number of medicinal plant } \\
\text { parts used in each case }\end{array}$ & $\begin{array}{c}\text { Percent } \\
\text { of total }\end{array}$ \\
\hline Oral & 157 & 44.9 \\
Dermal & 132 & 37.7 \\
Dermal, nasal and oral & 14 & 4.0 \\
Dermal and oral & 15 & 4.3 \\
Optical & 10 & 2.9 \\
Nasal & 9 & 2.6 \\
Ear & 8 & 2.3 \\
Vaginal & 2 & 0.6 \\
Dermal and nasal; nasal & 1 & 0.9 \\
and ear; nasal and oral & & \\
\hline
\end{tabular}

The preferences of leaves and roots to other plant parts could be attributed to ease of preparation, the presence of medicinally active secondary metabolites and accessibility at the required time in the same manner as described for western [34], southern [45-47,53,59-61], northern [41,58], central [62] and eastern [65] Ethiopia and other countries $[63,64]$. The use of leaves for medicinal purposes is less likely to be destructive especially relative to the use of roots. The latter is likely to have negative influence on the survival of the plant. Cultural practices and beliefs requiring digging up of three or seven plants to prepare just a single remedy have been recorded. In some cases three or five or seven pieces each had to be removed from the same or different individual plants and applied to cure the disease, which would likely be unfavorable to conservation. Preparations made from all parts, three and two plant parts for remedy formulations (few in our case) may endanger the species unless mechanisms for sustainable utilization are put in place. Single plant preparations are easier to extract the curative chemical compounds as reported by others [33]. However, mixtures are expected to be more effective due to the additive effects of the combination of plants by increasing the compounds that could act on different pathogens.

Higher frequencies of crushed forms could be related to the ease of preparation at any place, using stones at most, which could be done by most local community members. Informants asserted that medicinal plant parts crushed and soaked in water lead to effective and immediate response to health problems. Crushing came out as the most frequent preparation method in other works [38]. A prescription that required crushed roots of Asparagus africanus concocted with honey and stored for seven days in a bottle was used for the treatment of impotency. Healers explained that such a preparation helps to extract the active chemicals and this is analogous to the methods used in modern phytochemical and pharmacological extractions using different solvents in the laboratory. This hints at a fair understanding of the local people about the science behind the traditional practices of herbal remedy preparation and treatment. About $71 \%$ of the medicinal plant remedies were prepared from fresh plant material highlighting that live medicinal plants have to be found near homes for instant use. Most herbalists advised that fresh material are more effective for treatment than dried forms further elaborating that drying could easily distort the efficacy of the medicine, and that stored plant medicine is culturally less liked and was also reported by other researchers $[41,53,59]$ in Ethiopia. In modern herbal medicine, some secondary metabolites having active healing potentials are known to be quickly transformed to permanent compounds losing their healing power soon upon cutting $[5,8]$. The 


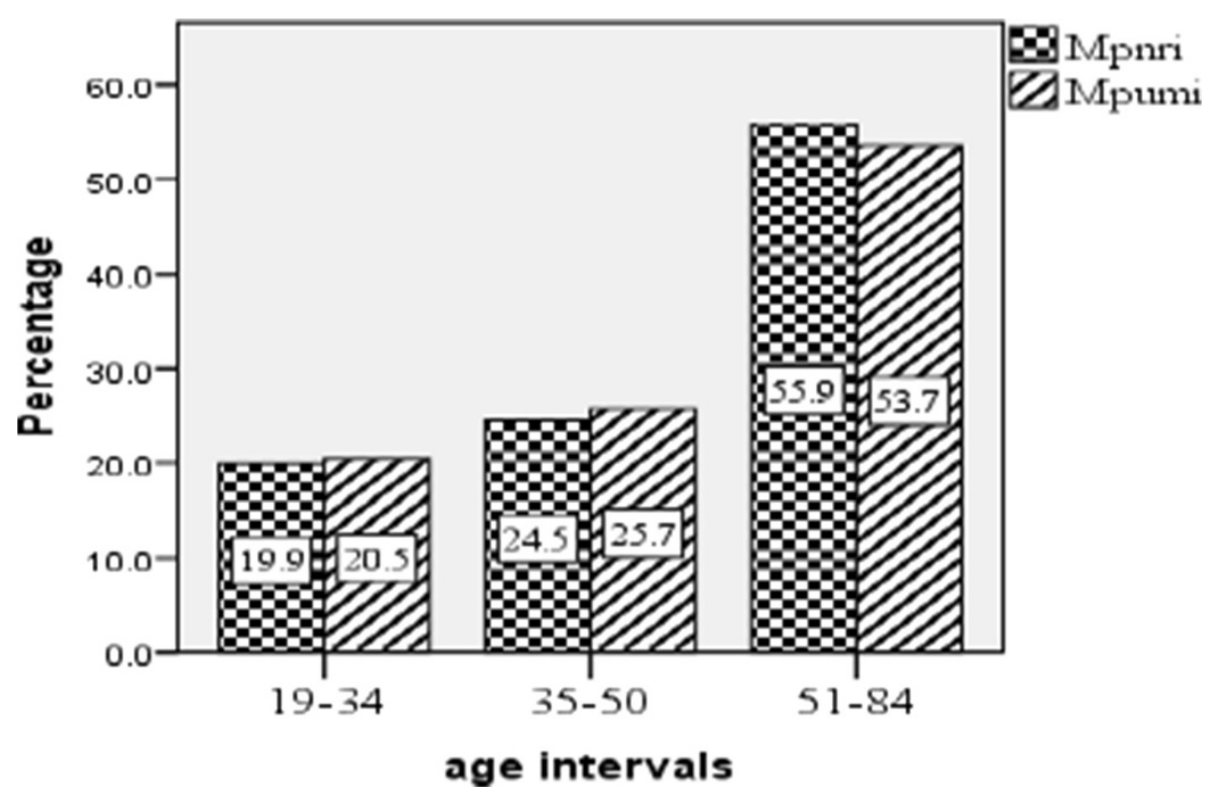

Figure 3 Variation of medicinal plants knowledge among the age groups of informants (Mpnri = Medicinal plant names reported by individuals, Mpumi $=$ Medicinal plant uses mentioned by individuals).

use of dried plants and stored remedies were reported by very few healers, who said that they use dried plant material when availability of fresh material is seasonal. Dependency on fresh material is likely to throw the species to serious threats as had been warned by other sources [39].

Informants affirmed that after the New Year holiday, preparations from the past year could not have the potential to cure ailments if not buried on the eve of the holiday upto the next day to respect the cultural and religious beliefs. The newly prepared remedies are believed to have active constituents such as (volatile oils and other phytochemical and pharmaceutical ingredients) and these could be lost progressively due to factors including temperature, oxidation and reduction. This tradition of collecting most of the medicinal plant materials once in a year has the merit of minimizing overharvesting. Various sources from central [33], western [34], southern [46,58-60], eastern [62] and northern
[41] Ethiopia proclaim that oral route is most frequent. Some sources $[33,34,58]$ that recorded measurements for remedies in a similar manner to ours noted the lack of precision and standardization as a drawback of the traditional herbal healthcare system. Additives are included in the medicines to minimize discomfort, improve the taste and reduce adverse effects such as vomiting and diarrhea, and enhance the efficacy and healing potential as explained by the informants. Mixing and using some medicinal plants with common foods and drinks is an easy way for effective treatment, particularly for children and facilitation of ingesting bitter tasting formulations as described in other sources $[33,34,58]$.

The recorded taboos and other ritual-like actions related to the collection, preparation and administration of traditional medicine are beliefs carried over generations in the study area in a similar manner to the research results reported from Bale [52] in southeast Ethiopia. The

Table 15 Priority ranking results of seven respondents on six factors perceived as threats to medicinal plants

\begin{tabular}{lccccccccc}
\hline \multicolumn{1}{c}{ Threatening factor } & \multicolumn{1}{c}{ Respondents (R1-R7) } \\
\cline { 2 - 9 } & $\mathbf{R}_{\mathbf{1}}$ & $\mathbf{R}_{\mathbf{2}}$ & $\mathbf{R}_{\mathbf{3}}$ & $\mathbf{R}_{\mathbf{4}}$ & $\mathbf{R}_{\mathbf{5}}$ & $\mathbf{R}_{\mathbf{6}}$ & $\mathbf{R}_{\mathbf{7}}$ & Total & $\mathbf{R a n k}^{\text {st }}$ \\
\hline Agricultural expansion & 6 & 6 & 6 & 5 & 5 & 6 & 4 & 38 & 32 \\
Overgrazing & 3 & 4 & 5 & 6 & 6 & 5 & 3 & $3^{\text {rd }}$ \\
Drought & 2 & 2 & 2 & 1 & 3 & 4 & 4 & 18 & $5^{\text {th }}$ \\
Fuelwood collection & 6 & 4 & 6 & 2 & 6 & 4 & 5 & 33 & $2^{\text {nd }}$ \\
Construction and building material & 1 & 3 & 5 & 1 & 1 & 2 & 3 & 15 & $6^{\text {th }}$ \\
Urbanization/Modernization & 4 & 6 & 5 & 2 & 3 & 4 & 5 & 29 & $4^{\text {th }}$ \\
\hline
\end{tabular}


Table 16 Results of preference ranking of five most threatened medicinal plants

\begin{tabular}{lccccccccc}
\hline Treating medicinal plant species & \multicolumn{10}{c}{ Respondents (R1-R7) } \\
\cline { 2 - 9 } & $\mathbf{R}_{\mathbf{1}}$ & $\mathbf{R}_{\mathbf{2}}$ & $\mathbf{R}_{\mathbf{3}}$ & $\mathbf{R}_{\mathbf{4}}$ & $\mathbf{R}_{\mathbf{5}}$ & $\mathbf{R}_{\mathbf{6}}$ & $\mathbf{R}_{\mathbf{7}}$ & Total & $\mathbf{R a n k}^{\text {r }}$ \\
\hline Cucumis ficifolius & 4 & 1 & 4 & 1 & 2 & 2 & 3 & 17 & $3^{\text {rd }}$ \\
Ficus carica & 3 & 2 & 1 & 2 & 3 & 2 & 3 & 16 & $4^{\text {th }}$ \\
Huernia macrocarpa & 2 & 3 & 3 & 4 & 4 & 4 & 5 & 25 & $2^{\text {nd }}$ \\
Solanum marginatum & 1 & 2 & 2 & 3 & 1 & 3 & 2 & 14 & $5^{\text {th }}$ \\
Withania somnifera & 5 & 4 & 5 & 5 & 5 & 5 & 4 & 33 & $1^{\text {st }}$ \\
\hline
\end{tabular}

interpretations correspond to healers' perceptions of medicine and disease treatment whose scientific verification awaits further studies.

Elderly members of the society (aged 51-85 years) had expectedly more knowledge on medicinal plants and their uses due to their long-lasting direct and regular contact with the forests and other plant resources. In contrast, the younger generation is more exposed to modern education and hence not interested in learning and practicing the ethnomedicinal wisdoms, which may affect the continuity of indigenous knowledge. Medicinal plant knowledge difference among age groups was also reported in other studies $[2,45,59,66]$ but one study from southern Ethiopia [47] deviated from this.

People living far away from forests (Asiba and Yifag Akababi) knew relatively fewer species than those residing near the forests (Washa Indiras, Kualla Yihuans and Kidanemhret) showing that contact with the plant resources helps to preserve and continue using the knowledge. Tibabosgie village being close to the forest reported less knowledge due to being more dependent on a few highly knowledgeable healers for their healthcare delivery. Mantogera village is close to Addis Zemen Town and the people have better access to modern medical system than traditional medicine. On the high land area of Aguat Mafsesha, the people live concentrated within a specific compatible area and intensive cultivation is the norm. Here, biodiversity is considerably reduced and the possibility of finding medicinal plants has been minimized.

The study confirmed that variation exists in species preferences among sites, partly due to the wide array of ecological niches within short distances. This is in turn expected to bring about differences in indigenous knowledge among informants of different sites. Similar trends have been reported in a study conducted in eastern central Ethiopia [38]. Though results indicated relative variations between town and rural villages, indigenous medicinal plant knowledge difference was hardly noticeable indicating that even town dwellers living close to forests keep considerable ethnobotanical knowledge as reported in other studies $[67,68]$.

It is no wonder that agriculture is the main culprit for the loss of medicinal plant habitats, vegetation and species because the communities in the study area depend more on mixed agriculture as their main economic activity with limited landholding and high human population [34,59,63,69]. Low living standards and lack of alternatives are major factors responsible for the decline of forest resources [14]. Cultivating the useful plants in homegardens is crucial, but conservation in the natural wild setting (in-situ) must also be considered since plants in their natural ecological area can grow at the limits of their potentials and provide the expected results including efficacy as medicine. Sustainable medicinal plant management and conservation are

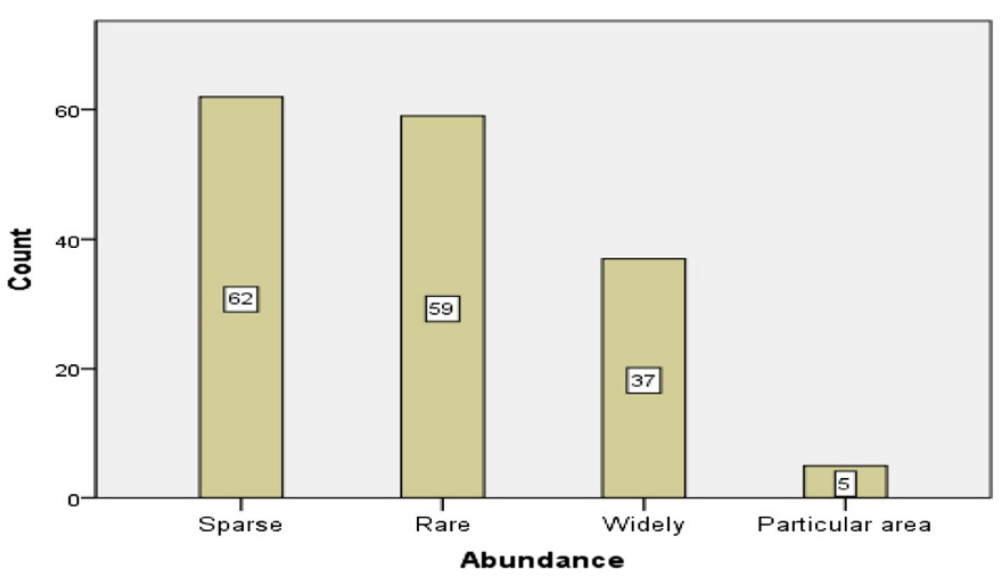

Figure 4 Current condition of medicinal plant species based on informant preferences. 
imperative for rural people's healthcare and community well-being. The importance and conservation purposes of church forests have previously been reported [70]. Likewise, the governmental plant nursery in Addis Zemen Town is used as a germplasm source for the forest as well as the surrounding areas. The nursery is engaged in raising seedlings of selected species that are distributed for reforestation and afforestation programmes, which needs further enhancement and scaling up.

\section{Conclusion}

The present study showed that Tara-gedam and Amba forests harbour a high diversity of medicinally useful plants and the people living in the area have a long history of plant use, and that of medicinal plants is exceptionally notable and culturally rooted in the area. Despite the gradual socio-cultural transformation, the inhabitants have retained remarkable knowledge of the plants and their uses. Difficulties in knowledge transfer and the resulting generation gap in knowledge are threatening the continuity of the medicinal plants and the indigenous knowledge on them. On the other hand, the study provided evidence that medicinal plants will continue to play an important role in the healthcare system in the study area, given support through conservation and education. Knowledge and herbal medical practices for the treatment of various ailments among both rural and urban people are major parts of their livelihoods and culture. The traditional knowledge of the use and conservation of these plants is still being transferred from generation to generation, but appeared to be aging. The problem of transfer of knowledge from the elders to the young generation probably arose following the introduction of modern education, religious, spiritual and culture-related factors. Therefore, it is not only essential to conserve such a wealth of information hidden among the local people but also to apply modern science and technology to meet the ever increasing requirements of humankind. Furthermore, conservation of these biological resources is very important because their sustainable use can generate higher levels of employment and income.

\section{Competing interests}

The authors declare that they have no competing interests.

\section{Authors' contributions}

All authors had significant intellectual contribution towards the design of the study, data collection and analysis and write-up of the manuscript. GC conducted the fieldwork, identified the plants, analyzed the data, wrote the draft manuscript and actively followed it up through revisions up to submission and after. ZA took part in plant identification, reviewed and edited the draft manuscript, provided comments and suggestions and checked its final version EK checked and confirmed the identification of the plants, provided comments and suggestions on the draft manuscript and checked its final version. All authors read and approved the final manuscript and agreed to its submission.

\section{Acknowledgements}

The authors acknowledge with thanks the Department of Biology (Addis Ababa University) for the financial support used to carry out the research and the technical staff of the National Herbarium (ETH) for availing plant identification facilities. The people of Libo Kemkem District, who positively responded to the research idea and shared their valuable knowledge and time with generosity and warm hospitality as well as the District offices for Agriculture and Rural Development, Health, Administration and Information Affairs and Traditional Medicinal Plants Association for their kind provision of data, general information and writing supportive letters during data collection. The authors also acknowledge with thanks professors Sebsebe Demissew and Sileshi Nemomissa for their valuable comments on the earlier version of the research output. The first author would also like to thank his family members for their multidimensional support during field data collection in particular.

\section{Author details}

${ }^{1}$ Department of Biology, Woldia University, P.O. Box 400, Woldia, Ethiopia. ${ }^{2}$ Department of Plant Biology and Biodiversity Management and The National Herbarium (ETH), Addis Ababa University, P.O. Box 3434, Addis Ababa, Ethiopia.

Received: 13 April 2014 Accepted: 19 October 2014

Published: 7 January 2015

\section{References}

1. Choudhary KK, Singh M, Pillai U: Ethnobotanical survey of Rajasthan. American-Eurasian. J Botany 2008, 1:38-45.

2. Cotton CM: Ethnobotany: Principles and Applications. Chichester: John Wiley and Sons Ltd; 1996.

3. Quanash N: Bicultural diversity and integrated healthcare in Madagascar. Nat Resour 1998, 30:18-22.

4. Martin GJ: Ethnobotany: A Method Manual. London: Chapman and Hall; 1995.

5. Bodeker G: Medicinal Plant Biodiversity and Local Healthcare: Sustainable use and Livelihood Development. The 17th commonwealth forestry conference, 2005, Colombo, Sri Lanka.

6. SCBD: Sustainable Forest Management, Biodiversity and Livelihoods: a Good Practice Guide. Montreal: IUCN; 2010.

7. Cunningham AB: People, Park and Plants use Recommendations for Multiple use Zones and Development Alternatives Around Bwindi: Impenetrable National Park Uganda. In People and Plants: Working Paper 4, pp. 18-25. Paris: UNESCO; 1996.

8. Awas T: Conservation of Medicinal Plants in Ethiopia. In The Proceedings of the First Medicinal Plant National Workshop Held in Addis Ababa, Ethiopia: June 30-July 2, 2003. Edited by Kelbessa U, Ayale A, Merga G. Addis Ababa: Ethiopian Health and Nutrition Research Institute; 2004:97-107.

9. Abebe $D$ : The role of medicinal plants in health care coverage of Ethiopia, the possible integration. In conservation and sustainable use of medicinal plants in Ethiopia. In Proceeding of National Workshop on Biodiversity Conservation \& Sustainable Use of Medicinal Plants in Ethiopia. Edited by Zewdu M, Demissie A. Addis Ababa: IBCR; 2001:6-21.

10. Lal R, Junior WF: Where Biodiversity, Traditional Knowledge, Health and Livelihoods Meet: Institutional Pillars for the Productive Inclusion of Local Communities. Brazil: International Policy Centre for Inclusive Growth; 2011.

11. Abebe D: Traditional medicine in Ethiopia: the attempts being made to promote it for effective and better utilization. SINET Ethiop J Sci 1986, 9:61-69.

12. Young J: Ethiopian Protected Areas, a 'Snapshot: A Reference Guide for Future Strategic Planning and Project; 2012.

13. Abiyu A, Gratzer G, Teketay D, Glatzel G, Aerts R: Epiphytic recruitment of Schefflera abyssinica (A. Rich) Harms. and the role of microsites in affecting tree community structure in remnant forests in northwest Ethiopia. SINET Ethiop J Sci 2013, 36:41-44.

14. Zegeye $H$, Teketay $D$, Kelbessa E: Diversity and regeneration status of woody species in Tara-gedam and Abebaye forests, Northwestern Ethiopia. J Forestry Res 2011, 22:315-328.

15. Teshome $\mathrm{W}$ : Impacts of urbanization on the traditional medicine of Ethiopia. J Anthro 2005, 8:43-52.

16. LKWISO: Agro-Climatic Zones of Libo Kemkem Woreda. Addis Zemen: Libo Kemkem Woreda Information Source Office; 2010.

17. LKWCTO: Libo Kemkem Woreda Cultural and Tourism Records. Libo Kemkem: Woreda Culture and Tourism Office; 2010.

18. CSA: The 2007 Population and Housing Census for Ethiopia, Statistical Report Results at Country Level. Addis Ababa: Central Statistical Authority; 2007 
19. LKWHO: Human Health Related Information. Addis Zemen: Libo Kemkem Woreda Health control office; 2010.

20. LKWAHO: Animal Related Information in Libo Kemkem Woreda. Addis Zemen: Libo Kemkem Woreda Animal Health Organization; 2010.

21. Cunningham AB: Applied Ethnobotany: People, Wild Plant use and Conservation. People and Plant Conservation Manual. London: Earthscans Publication Ltd; 2001

22. Balick MJ, Cox PA: Plants, People and Culture: Science of Ethnobotany. New York: Scientific American Library; 1996

23. Edwards S, Demissew S, Hedberg I (Eds): Flora of Ethiopia and Eritrea Hydrocharitaceae to Arecaceae Volume 6. Ethiopia: Department of Systematic Botany, Uppsala University, Sweden and The National Herbarium, Addis Ababa University; 1997

24. Edwards S, Tadesse M, Demissew S, Hedberg I (Eds): Flora of Ethiopia and Eritrea. Magnoliaceae to Flacourtiaceae Volume 2 Part 1. Ethiopia: Department of Systematic Botany, Uppsala University, Sweden and The National Herbarium, Addis Ababa University; 2000.

25. Edwards S, Tadesse M, Hedberg I (Eds): Flora of Ethiopia and Eritrea. Canellaceae to Euphorbiaceae Part 2 Volume 2. Ethiopia: Department of Systematic Botany, Uppsala University, Sweden and The National Herbarium, Addis Ababa University; 1995

26. Hedberg I, Edwards S (Eds): Flora of Ethiopia and Eritrea. Poaceae (Graminae) Volume 7. Ethiopia: Department of Systematic Botany, Uppsala University, Sweden and The National Herbarium, Addis Ababa University; 1995.

27. Hedberg I, Edwards S (Eds): Flora of Ethiopia and Eritrea. Pittosporaceae to Araliaceae Volume 3. Ethiopia: Department of Systematic Botany, Uppsala University, Sweden and The National Herbarium, Addis Ababa University; 1989.

28. Hedberg I, Edwards S, Nemomissa S (Eds): Flora of Ethiopia and Eritrea. Apiaceae to Dipsacaceae Volume 4 part 1. Ethiopia: Department of Systematic Botany, Uppsala University, Sweden and The National Herbarium, Addis Ababa University; 2003.

29. Hedberg I, Friis I, Edwards S (Eds): Flora of Ethiopia and Eritrea. Asteraceae Part 2 Volume 4. Ethiopia: Department of Systematic Botany, Uppsala University, Sweden and The National Herbarium, Addis Ababa University; 2004.

30. Hedberg I, Friis I, Person E (Eds): Flora of Ethiopia and Eritrea. Lycopodiaceae to Pinaceae. Volume 1. Ethiopia: Department of Systematic Botany, Uppsala University, Sweden and the National Herbarium, Addis Ababa University; 2009

31. Hedberg I, Kelbessa E, Edwards S, Demissew S, Persson E (Eds): Flora of Ethiopia and Eritrea. Plantaginaceae Volume 5. Ethiopia: Department of Systematic Botany, Uppsala University, Sweden and The National Herbarium, Addis Ababa University; 2006.

32. Heinrich M: Ethnobotany and its role in drug development. Phytother Res 2000, 14:479-488.

33. Gedif T, Hahn HJ: Herbalists in Addis Ababa and Butajira, Central Ethiopia: Mode of service delivery and traditional pharmaceutical practice. Ethiop J Health Dev 2002, 16:191-197.

34. Yirga G, Zeraburk S: Ethnobotanical study of traditional medicinal plants in Gindeberet District, western Ethiopia. Mediterr J Social Sci 2011, 2:49-54.

35. Fassil H: Beyond plants, professionals and parchments: the role of homebased medicinal plant use and traditional health knowledge in primary health care in Ethiopia. Ethnobotany Res App/ 2005, 3:037-049.

36. FAO: World Reference Base for Soil Resources 2006: A Framework for International Classification, Correlation and Communication. World Soil Resources Reports No. 103. Rome: FAO; 2006.

37. Corbeels M, Shiferaw A, Haile M: Farmers' Knowledge of Soil Fertility and Local Management Strategies in Tigray, Ethiopia: Managing Africa's Soils No. 10. Nottingham: Russell Press; 2000.

38. Bahru T, Asfaw Z, Demissew S: Variation of indigenous botanical knowledge versus social characteristics between the Afar and Oromo nations in and around the semi-arid Awash National Park, Ethiopia. Ethiop J App/ Sci Technol 2011, 2:75-90.

39. Megersa M, Asfaw Z, Kelbessa E, Beyene A, Woldeab B: An ethnobotanical study of medicinal plants in Wayu Tuka District, East Welega Zone of Oromia Regional State, West Ethiopia. J Ethnobiol Ethnomed 2013, 9:68.

40. Lulekal E, Asfaw Z, Kelbessa E, Van Damme P: Ethnomedicinal study of plants used for human ailments in Ankober District, North Shewa Zone, Amhara Region, Ethiopia. J Ethnobiol Ethnomed 2013, 9:63.

41. Teklay A, Abera B, Giday M: An ethnobotanical study of medicinal plants used in Kilte Awulaelo District, Tigray Region of Ethiopia. J Ethnobiol Ethnomed 2013, 9:65.

42. Hailemariam T, Demissew S, Asfaw Z: An ethnobotanical study of medicinal plants used by local people in the lowlands of Konta Special Woreda, southern Nations, Nationalities and Peoples Regional State, Ethiopia. J Ethnobiol Ethnomed 2009, 5:26.
43. Giday M, Asfaw Z, Woldu Z, Teklehaymanot T: Medicinal plant knowledge of the Bench ethnic group of Ethiopia: an ethnobotanical investigation. J Ethnobiol Ethnomed 2009, 5:34.

44. Regassa R: Assessment of indigenous knowledge of medicinal plant practice and mode of service delivery in Hawassa city, southern Ethiopia. J Med Plants Res 2013, 9:517-535.

45. Awas T, Demissew S: Ethnobotanical study of medicinal plants in Kafficho people, Southwestern Ethiopia. In Proceedings of the 16th International Conference of Ethiopian Studies. Edited by Ege S, Aspen H, Teferra B, Bekele ST; 2009.

46. Mesfin F, Demissew S, Teklehaymanot T: An ethnobotanical study of medicinal plants in Wonago Woreda, SNNPR, Ethiopia. J Ethnobiol Ethnomed 2009, 5:28.

47. Adefa MS, Getaneh SA: Medicinal plants biodiversity and local Healthcare management system in Chencha District; Gamo Gofa, Ethiopia. J Pharmacogn Phytochem 2003, 2:1

48. Hedberg I, Friis I, Edwards S, Persson E, Tadesse M, Demissew S, Phillips S (Eds): Flora of Ethiopia and Eritrea Volume 8: General Part and Index to Volumes 1-7. Addis Ababa: Uppsala University, Department of Systematic Botany and National Herbarium; 2009

49. Birhanu Z: Traditional use of medicinal plants By the Ethnic Groups of Gondar Zuria District, North-western Ethiopia. J Nat Remed 2013, 13:1.

50. Agize M, Demissew S, Asfaw Z: Ethnobotany of medicinal plants in Loma and Gena bosa districts (woredas) of dawro zone, southern Ethiopia. Topclass J Herbal Med 2013, 2:194-212.

51. Zenebe G, Zerihun M, Solomon Z: An ethnobotanical study of medicinal plants in Asgede Tsimbila district, Northwestern Tigray, northern Ethiopia. Ethnobotany Res Appl 2012, 10:305-320.

52. Yineger $\mathrm{H}$, Kelbessa $\mathrm{E}$, Bekele $\mathrm{T}$, Lulekal $\mathrm{E}$ : Plants used in traditional management of human ailments at Bale Mountain National Park, Southeastern Ethiopia. J Med Plant Res 2008, 2:132-153.

53. Yineger $\mathrm{H}$, Yewhalaw D: Traditional medicinal plant knowledge and use by local healers in Sekoru Woreda, Jimma Zone, Southwestern Ethiopia. J Ethnobiol Ethnomed 2007, 3:24.

54. Teklehaymanot T, Giday M: Ethnobotanical study of medicinal plants used by people in Zegie Peninsula, Northwestern Ethiopia. J Ethnobiol Ethnomed 2007, 3:12

55. Flatie T, Gedif T, Asres K, Gebre-Mariam T: Ethnomedical survey of Berta ethnic group Assosa Zone, Benishangul-Gumuz regional state, mid-west Ethiopia. J Ethnobiol Ethnomed 2009, 5:14.

56. Gidey M: An ethnobotanical study of medicinal plants used by the Zay people in Ethiopia. CBM s Skriftserie 2001, 3:81-99.

57. Belayneh A, Bussa FN: Ethnomedicinal plants used to treat human ailments in the prehistoric place of Harla and Dengego valleys, eastern Ethiopia. J Ethnobiol Ethnomed 2014, 10:18.

58. Mesfin K, Tekle G, Tesfay T: Ethnobotanical study of traditional medicinal plants used by indigenous people of Gemad District, Northern Ethiopia. J Med Plants Stud 2013, 1:4.

59. Abera B: Medicinal plants used in traditional medicine by Oromo people, Ghimbi District, Southwest Ethiopia. J Ethnobiol Ethnomed 2014, 10:40.

60. Tolossa K, Debela E, Athanasiadou S, Tolera A, Ganga G, Houdijk Jos GM: Ethno-medicinal study of plants used for treatment of human and livestock ailments by traditional healers in South Omo, Southern Ethiopia. J Ethnobiol Ethnomed 2013, 9:32.

61. Lulekal E, Kelbessa E, Bekele T: An ethnobotanical study of medicinal plants in Mana Angetu District, Southeastern Ethiopia. J Ethnobiol Ethnomed 2008, 4:10.

62. Adefa MS, Abraha BT: Ethnobotanical survey of traditional medicinal plants in Tehuledere District, South Wollo, Ethiopia. J Med Plants Res 2011, 5:6233-6242.

63. Kumar N, Nautiyal S: An Inventory of medicinal wealth of Jhil-Mil Jheel conservation reserve. Int J Herbal Med 2013, 1:2

64. Asiimwea S, Namutebib A, Borg-Karlssonc AK, Mugishaa MK, Oryem-Origa H: Documentation and consensus of indigenous knowledge on medicinal plants used by the local communities of western Uganda. J Nat Prod Plant Resour 2014, 4:34-42.

65. Belayneh A, Asfaw Z, Demissew S, Bussa NF: Medicinal plants potential and use by pastoral and agro-pastoral communities in Erer Valley of Babile Wereda, Eastern Ethiopia. J Ethnobiol Ethnomed 2012, 8:42.

66. Teklehymanot T, Giday M, Medihin G, Mekonnen Y: Knowledge and use of medicinal plants by people around Debrelibanos Monastery in Ethiopia. J Ethnopharmacol Ethnomed 2007, 111:271-283.

67. Mengistu $\mathrm{F}$, Hager $\mathrm{H}$ : Wild edible fruit species cultural domain, informant species competence and preference in three Districts of Amhara Region, Ethiopia. Ethnobotany Res Appl 2008, 6:487-502. 
68. Leonard W, Wilkie D: Indigenous Knowledge and Consumption of Wild Plants: A Comparative Study of two Tsimane Villages in the Bolivian Amazon. Garcia: Victoria Reyes; 2008.

69. Tedla S, Lemma K: Environmental Management in Ethiopia: Have the National Conservation Plans Worked. Environmental Forum Publications Series no.1. Addis Ababa, Ethiopia: OSSREA; 1998.

70. Dagnachew M: The Role of the Ethiopian Orthodox Tewahedo Church in Preserving Trees and Woodlands. Addis Ababa: Ethiopian Orthodox Tewahedo Church; 2001.

doi:10.1186/1746-4269-11-4

Cite this article as: Chekole et al.: Ethnobotanical study of medicinal plants in the environs of Tara-gedam and Amba remnant forests of Libo Kemkem District, northwest Ethiopia. Journal of Ethnobiology and Ethnomedicine 2015 11:4

\section{Submit your next manuscript to BioMed Central and take full advantage of:}

- Convenient online submission

- Thorough peer review

- No space constraints or color figure charges

- Immediate publication on acceptance

- Inclusion in PubMed, CAS, Scopus and Google Scholar

- Research which is freely available for redistribution 